\title{
D. LEHMANN
}

\section{Quelques propriétés des connexions induites, et fibrés à groupe structural variable en géométrie d'ordre supérieur}

Mémoires de la S. M. F., tome 16 (1968)

<http://www.numdam.org/item?id=MSMF_1968_16_1_0>

(C) Mémoires de la S. M. F., 1968, tous droits réservés.

L'accès aux archives de la revue «Mémoires de la S. M. F. » (http://smf. emath.fr/Publications/Memoires/Presentation.html) implique l'accord avec les conditions générales d'utilisation (http://www.numdam.org/conditions). Toute utilisation commerciale ou impression systématique est constitutive d'une infraction pénale. Toute copie ou impression de ce fichier doit contenir la présente mention de copyright.

\section{Numdam}




\title{
BULLETIN
}

DE LA

\section{SOCIÉTÉ MATHÉMATIQUE DE FRANCE}

PUBLIÉ

AVEC LE CONCOURS DU CENTRE NATIONAL DE LA RECHERCHE SCIENTIFIQUE

\section{SUPPLÉMENT au numéro de DECEMBRE 1968}

\author{
MÉMOIRE N 16
}

Bull. Sor. math. France.

Mémoire 16, 1968, $128 \mathrm{p}$.

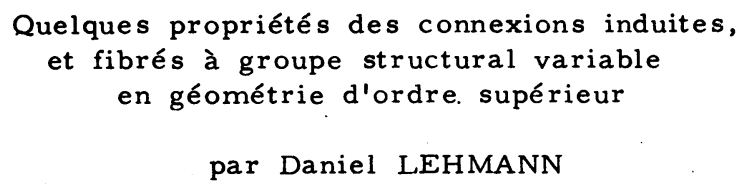

SOCIÉTÉ MATHÉMATIQUE DE FRANCE

11, rue Pierre et Marie Curie, 75 - PARIS $5^{e}$

Publication trimestrielle 
SUPPÍ́MENTS au Bulletin de la Société mathématique de France.

- Les "Comptes rendus des séances", qui avaient paru annuellement de 1911 à 1938, ne sont plus disponibles séparément, mais sont tous incorporés, année par année, dans la réimpression du Bulletin de la Société mathématique de France, tome 39 (1911) à 66 (1938), y compris, pour chacune des années 1911, 1921, 1922, 1923 et 1924 (séances du "Cinquantenaire" et séances ordinaires de 1924), les tables qui n'existaient pas à l'origine.

Les autres suppléments ci-après sont disponibles séparément :

- 1939. - Conférences de la Réunion internationale des mathématiciens, tenue à Paris en juillet 1937.

- "Mémoires" :

1. FORT (Jacques). - Contribution à l'étude des éléments tertiaires et isotypiques dans les modules et les ( $\zeta)$-algèbres (Thèse).

2. GIRAUD (Jean). - Méthode de la descente.

3. GRILLET (Pierre-Antoine). - Homomorphismes principaux de tas et de groupoIdes (Thèse).

4. BERTRANDIAS (Françoise). - Ensembles remarquables d'adèles algébriques (Thèse).

5. BERTRANDIAS (Jean-Paul). - Espaces de fonctions bornées et continues en royenne asymptotique d'ordre $p$ (Thèse).

6. VO-KHAC Khoan. - Etude des fonctions quasi stationnaires et de leurs Applications aux équations différentielles opérationnelles (Thèse).

7. BERNAT (Pierre). - Sur le corps enveloppant d'une algèbre de Lie résoluble.

8. MALLIAVIN-BRAMERET (Marie-Paule). - Largeurs d'anneaux et de rodules (Thèse).

9. RENAUIT (Guy). - Etude des sous-modules compléments dans un module (Thèse).

10. ZINN-JUSTIN (Nicole). - Dérivatiuns dans les corps et anneaux de caractéristique p (Thèse).

11. BERTIN (Jean-Etienne). - Variété de Picard de type linéaire commutatif (Thèse).

12. AUBIN (Jean-Pierre). - Approximation des espaces de distribution et des opérateurs différentiels (Thèse).

13. DEUTSCH (Mme Nimet). - Interpolation dans les espaces vectoriels topologiques locelement convexes (Thèse).

14. ROBERT (Plerre). - Sur I'axiomatique des systèmes générateurs, des rangs, ... Applications à certains problèmes combinatoires (Thèse).

15. FOUQUES (Alfred). - Systèmes de a-idéaux dans un demi-groupe non commutatif (Thèse).

16. LEHMANN (Daniel). - Quelques propriétés des connexions induites, et fibrés à groupe structural variable en géométrie d'ordre supérieur (Thèse).

() Tous droits réservés

Société mathématique de France. 
Bull. Soc. math. France,

Mémoire 16, 1968, $128 \mathrm{p}$.

lère partie : Quelques propriétés des connexions induites

2ème partie : Fibrés à groupe structural variable en Géométrie d'ordre supérieur

par

Daniel LEHMAIM (*)

-:::-:::-

TABLE DES MATIERES

PREMIERE PARTIE

Pages

Introduction $\ldots \ldots \ldots \ldots \ldots \ldots \ldots \ldots \ldots \ldots \ldots \ldots \ldots \ldots \ldots \ldots \ldots \ldots \ldots \ldots \ldots \ldots \ldots \ldots$

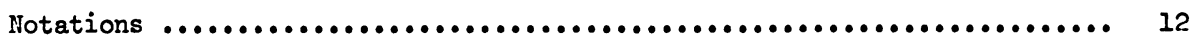

Chapitre I : Rappels sur les fibrés vectoriels réels différentiebles 14

A - Opérations sur les fibrés vectoriels ........................ 15

B - Formes différentielles à valeurs dans un fibré vectoriel ....... 21

C - Connexions ........................................... 25

D - Différentiation extérieure ............................. 29

(*) Thèse Sc. math. Paris, 1966 
A - Plongements d'espaces fibrés. Formes de plongement.

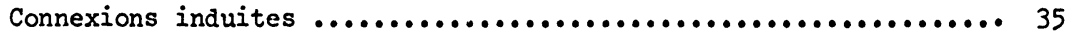

E - Connexions sur un produit fibré .......................... 40

C - Premiers exemples $\ldots \ldots \ldots \ldots \ldots \ldots \ldots \ldots \ldots \ldots \ldots \ldots \ldots \ldots \ldots \ldots \ldots \ldots \ldots \ldots \ldots \ldots$

10) G-connexions ..................................... 43

$\left.2^{\circ}\right)$ Connexions de Cartan ................................. 46

$\left.3^{\circ}\right)$ Espaces de Minkowski .................................. 49

$\left.4^{\circ}\right)$ Espaces homogènes réductifs ........................ 52

Chapitre III : Connexions induites sur les fibrés vectoriels 54

A - Projections .......................................... 55

B - Transcription en termes de fibrés principaux ................6 6I

C - Connexions canoniques sur les variétés de Stiefel généralisées ... 69

D - Géométrie des sous-variétés ............................ 72

Chapitre IV : Extensions triviales d'une connexion $\quad 77$

A - Extensions principales triviales ........................ 78

B - Extensions vectorielles triviales ....................... 81

C - Comparaison entre extensions triviales vectorielles et principales 88

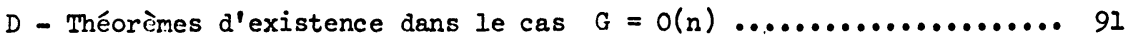

Bibliographie $\ldots \ldots \ldots \ldots \ldots \ldots \ldots \ldots \ldots \ldots \ldots \ldots \ldots \ldots \ldots \ldots \ldots \ldots \ldots \ldots \ldots \ldots \ldots \ldots \ldots$ 
DEUXIEME PARTIE

Introduction

Chapitre I : Espaces fibrés admettant_un

"espace structural fibré en groupe"

A - Espaces fibrés presque principaux 107

B - Fibrés modelés sur un fibré presque principal 109

C - Sous-fibrés presque principaux

Chapitre II : Prolongements holonomes des espaces fibrés

Connexions holonomes d'ordre supérieur

A - Prolongement holonome d'ordre $m$ d'un fibré principal

118

B - Prolongement holonome d'ordre $m$ d'un fibré associé ........... 119

C - Connexions holonomes d'ordre m sur $\&$..................... 120

Chapitre III : Généralisations aux of -prolongements 

PREMIERE PARTIE

Quelques propriétés des

connexions induites 
On connaît le théorème de $\mathrm{J}$. MASH affirmant la possibilité de plonger isométriquement toute variété riemannienne dans un espace euclidien de dimension suffisamment grande (problème đéjà considéré localement par E. CARTAN et M. JEANNET). Il en résulte que la connexion de LEVI-CIVITA d'une variété riemannienne peut toujours être induite (en un sens à préciser) par une certaine connexion plate.

Nous nous sommes intéressés ici à la généralisation d'une telle situation, et montrons en particulier, que dans le cas d'un groupe structural compact, toute connexion peut être induite par une connexion à courbure nulle. Le principe de la démonstration consiste à étudier le cas particulier de la connexion canonique d'une variété de Stiefel généralisée, et à utiliser le théorème de M.S. NARASIMHAN et S. RAMANAN affirmant le caractère universel de cette connexion canonique dans le cas du groupe orthogonal.

L'essentiel de ce travail est localisé dans les chapitres III (paragraphes B et C) et IV.

\section{Chapitre I : Rappels sur les fibrés vectoriels réels différentiables}

Soient $E \longrightarrow U$ un fibré vectoriel de base $U$ et fibre type $M$, $x$ un point de $U$, et $e$ un élément de $E_{x}$. Dans la pratique, on sait qu'il est souvent commode d'exprimer e par ses coordonnées relativement à un repère de $E_{x}$ : celà revient à se donner un isomorphisme d'espaces vectoriels $M \stackrel{\xi}{\simeq} E_{x}$ (c'est-à-dire un point $\xi$ de la fibre $P_{X}$ du fibré principal $P$ associé à $E$ ) et à définir e par son image $\xi^{-I}(e)$ dans $M$.

Le choix d'un repère $\xi$ particulier étant généralement considéré comme peu élégant, on préfèrera n'en fixer aucun et définir e par la fonction $\varphi: P_{x} \longrightarrow M$ qui, ̀̀ $\xi$, associe $\xi^{-I}$ (e) (étant bien entendu que cette fonction, qui vérifie

$$
\varphi(\xi B)=g^{-I} \varphi(\xi) \quad \forall \xi \in P_{x}, \quad \forall B \in G L(M),
$$


est entièrement déterminée par sa valeur en un seul point). Il revient au même de considérer $E_{x}$ comme l'espace quotient de $M \times P_{x}$ par la relation d'équivalence $(m, \xi) \sim\left(B-\frac{I}{m}, \xi g\right) \quad \forall B \in G L(M)$ (on reconnaît l'origine de la définition d'un fibré de fibre type donnée, modelé sur un fibré principal donné).

Ainsi une théorie de Géométrie différentielle aura généralement deux expressions :

- l'une, en termes de fibrés vectoriels (l) , sera souvent plus géóétrique,

- l'autre, en termes de fibrés principaux, correspondra simplement à la façon moderne d'exprimer la théorie avec des coordonnées.

Dans ce premier chapitre, nous nous proposons essentiellement de rappeler quelques définitions et détails techniques relatifs à cette dualité d'expression. Pour toute démonstration, on pourra se référer en particulier à

BERNARD [1], KOSZUL [10], LICHNEROWICZ [16] et NOMIZU [20].

Chapitre II : Connexions induites sur les fibrés principaux

Toute connexion sur un G-fibré principal $Q$ induit de façon naturelle une connexion sur tout sous-fibré principal de groupe $H$, pourvu que $G / H$ soit un espace homogène réductif (par. A). On définit aussi les formes de plongement.

La donnée d'une connexion sur le produit fibré $P P^{\prime}$ de deux fibrés principaux $P$ et $P^{\prime}$ de même base $U$, est équivalente aux données d'une connexion sur $P$ et $d^{\prime}$ une connexion sur $P^{\prime}$ (par. $\left.B\right)$.

Dans chacun des exemples étudiés au $\$ \mathrm{C}$, on s'efforcera d'interpréter géométriquement les formes de plongement :

- dans le cas d'une G-structure définie par un tenseur $t$, la forme de plongement, relative à une connexion linéaire, définit la dérivée covariante de $t$ par rapport à cette connexion.

- dans le cas d'une connexion de CARTAN, la forme de plongement est la forme de soudure de l'espace de repères associé à cette connexion.

(1) ou, plus généralement, en termes de fibrés non principaux 
- pour un espace de MINKOWSKI, la forme de plongement mesure "l'écart" de cet espace avec un espace euclidien.

- pour un espace homogène réductif, la forme de plongement est la forme fondamentale d'espace de repères sur le H-fibré principal $G \longrightarrow G / H$.

Les résultats des $\S A$ et $B$ sont essentiellement contenus dans [23] et ont été retrouvés indépendamment dans [5], [11] et [18].

Pour le $5 \mathrm{C}$, voir aussi (liste non exhaustive) :

- BERNARD [1], GOETZ [6] et LICHNEROWICZ [16] p. 237 pour I'exemple 1,

- EHRESMANN [4], LICHNEROWICZ [16], et NOMIZU p. $73 "$ " 2 ,

- gUiLLEMAIN [7] " " 3 ,

- LICHNEROWICZ [17], p. 48 et NOMIZU [2I] " " 4 ,

Chapitre III : Connexions induites sur les fibrés vectoriels

Après une étude géométrique des lois de dérivation sur les fibrés vectoriels qui sont somme de WHITNEY de deux sous-fibrés vectoriels ( $\$ A$ ), nous montrerons que sa transcription en termes de fibrés principaux ( $\$$ B) fait intervenir des situations qui forment successivement :

- un cas particulier de II-A (où $G$ est ici un sous-groupe de $G L(n, R)$ et $H=G \cap(G L(k, R) \times G L(n-k, R))$

- et un cas particulier de II-B (où $H$ est le produit direct $H^{\prime} \times H^{\prime \prime}$ avec $H^{\prime}=G \cap(G L(k, R) \times\{I\})$ et $H^{\prime \prime}=G \cap(\{I\} \times G L(n-k, R))$.

$\mathrm{Au}$ \& $\mathrm{C}$, nous expliquerons comment la connexion canonique d'une variété de Stiefel s'interprète dans ce cadre, donnant de cette connexion une nouvelle définition plus géométrique que les anciennes (cf. S. KOBAYASHI [9], NARASIMHAN et RAMANAN [19] et TAKIZAWA [23]).

$\mathrm{Au}$ \& $\mathrm{D}$, nous étudierons l'exemple important, convenablement généralisé, des sous-variétés d'une variété pseudo-riemannienne. 
Chapitre IV : Extensions triviales d'une connexion

L'espace homogène $G / H$ étant supposé réductif, et un supplémentaire $d b$ de $\mathrm{H}$ dans $G$ (stable par ad(H) ) étant donné, nous avons déjà montré que la connexion canonique $\omega_{0}$ sur le H-fibré principal $P_{0}: G \longrightarrow G / H$ est induite par la connexion naturelle à courbure nulle du G-fibré trivial $G \times G / H \longrightarrow G / H$ obtenu à partir de $P_{0}$ par extension du groupe structural. On verra que $\omega_{0}$ joue en fait un rôle universel parmi toutes les connexions sur un H-fibré principal $P$, qui possèdent une telle "G-extension triviale" ( $5 \mathrm{~A})$.

Nous avons déjà montré que la connexion canonique $D^{k}$ du fibré vectoriel $E_{k}^{n} \longrightarrow G_{k, n-k}$ est obtenue par projection (parallèlement à $E_{n-k}^{n}$ ) de la connexion naturelle à courbure nulle du fibré vectoriel trivial $R^{n} \times G_{k, n-k} \longrightarrow G_{k, n-k}$ (cf. III-C). En fait, $D^{k}$ est universelle parmi toutes les connexions $D$ sur un fibré vectoriel $E$, possédant une telle "n-extension triviale". On retrouve ainsi, de façon particulièrement simple, un résultat de S. KOBAYASHI [9] relatif aux variétés riemanniennes de dimension $k$ immergées isométriquement dans l'espace euclidien $R^{n} \quad(\S B)$.

Interprétée en termes de fibrés principaux dans le cas où $G C$ GL $(n, R)$ et $H=H^{\prime} \times H^{\prime \prime}=G \cap(G L(k, R) \times G L(n-k, R))$, l'existence d'une n-extension triviale de $D$ est une propriété moins forte que l'existence d'une G-extension triviale de la connexion w correspondant à $D$ sur le H'-fibré principal associé. Toutefois, localement, ces deux propriétés sont équivalentes (\$C).

Dans le cas $G=O(n)$, un théorème de NARASIMHAN et RAMANAN [19] prouvant le caractère universel de la connexion canonique $D^{k}$ du fibré universel $E_{k}^{n} \longrightarrow G_{k, n-k}$ (pour $n$ assez grand) permet de montrer l'existence d'une n-extension triviale de toute connexion à groupe d'holonomie compact. Réciproquement, prouvant directement dans certains cas l'existence d'une n-extension triviale, on peut améliorer le résultat de [19] en abaissant $n$ (5D).

Une partie du contenu de ce chapitre est résumée dans [13] et [14] . 


\section{Notations}

Les variétés, espaces fibrés et applications considérés seront toujours supposées différentiables de classe $c^{\infty}$ (et exceptionnellement analytiques).

Si W est une variété, on notera :

$T_{x}(W)$ l'espace vectoriel tangent $\searrow W$ en un point $x$ de $W$,

$D(W)$ l'anneau des fonctions différentiables sur $W$ à valeurs réelles,

$T(W)$ le fibré vectoriel tangent $\Xi$ $W$,

$T(W)$ le $D(W)$ - module des champs de vecteurs différentiables sur $W$.

Plus généralement, si $\mathrm{E} \longrightarrow W$ est un fibré vectoriel différentiable, on notera $\underset{m}{E}$ le $D(W)$ - module des sections différentiables de $E$.

Si $U \stackrel{f}{\longrightarrow} V$ est un morphisme de variété, si a est une forme différentielle sur $V$.et si $A \longrightarrow V$ est un fibré (principal ou vectoriel) au-dessus de $\mathrm{V}$, on notera :

$A_{x}$ la fibre de $A$ en un point $x$ de $V$

Af le fibré de base $U$ image réciproque de $A$ par $f\left((A f)_{x}=A_{f(x)}\right)$

af la forme sur $U$ image réciproque de $\propto$ par $f$.

Si f est un plongement régulier de $U$ dans $V$, on écrira parfois $A \mid U$ (resp. $\alpha \mid U$ ) au lieu de Af (resp. $\alpha f$ ).

Si $G$ est un groupe de Lie, on notera $G$ son algèbre de Lie.

Si H est un sous-groupe de lie de $G$, et si dí désigne un supplémentaire de $\underline{H}$ dans $G$, on notera $a_{\underline{H}}$ et ad , les projections sur $\underline{H}$ et sur do (parallèlement à db et $\underline{H}$ ) d'un élément a de $\underline{G}$. 
Si $P \longrightarrow U$ est un fibré principal différentiable de groupe structural G (on dira en abrégé : un "G fibré principal") et si $R: G \longrightarrow$ A ut $M$ est une représentation de $G$ come groupe d'automorphismes d'une certaine variété différentiable $M$, on notera $M_{\mathscr{Q}(G)}[P]$ (ou $M_{G}[P]$ s'il n'y a pas d'ambigüité sur $R$ ) le fibré (1) de fibre type M modelé sur $P$.

Si $\mathrm{P}^{\prime} \longrightarrow U$ est un $\mathrm{G}^{\prime}$-fibré principal différentiable, on notera $P \otimes P^{\prime}$ le produit fibré de $P$ et $P^{\prime}$ : c'est un $G$ × $G^{\prime}$ fibré principal différentiable de base $U$, défini comme la sous-variété de $P \times P^{\prime}$ telle que $\left(P \otimes P^{\prime}\right)_{x}=P_{x} \times P_{x}^{\prime} \quad\left(G \times G^{\prime}\right.$ opérant à droite de façon naturelle $)$.

(1) Si M est munie d'une structure de type $S$ plus fine que celle de variété différentiable, et si $G$ préserve cette structure quand il opère sur $M$ par $Q$, chaque fibre de $M Q[P]$ admet une structure naturelle de type $S$ isomorphe (non canoniquement) à celle de $M$ - Par exemple, si $M$ est un espace vectoriel réel de dimension finie, et si $R$ est une représentation linérire, $M \mathscr{R}(G)[P]$ est un fibré vectoriel réel différentiable. 
A - Opérations sur les fibrés vectoriels

B - Formes différentielles à valeurs dans un fibré vectoriel

C - Connexions

D - Différentiation extérieure 
A - Opérations sur les fibrés vectoriels

Tous les fibrés vectoriels considérés seront supposés réels et différentiables.

Soient $\mathrm{P} \longrightarrow \mathrm{U}$ (resp. $\mathrm{P}^{\prime} \longrightarrow \mathrm{U}$ ) un fibré principal différentiable de groupe structural $G$ (resp. $\left.G^{\prime}\right), M\left(\right.$ resp. $\left.M^{\prime}\right)$ un espace vectoriel réel et $R$ (resp. $Q^{\prime}$ ) une représentation linéaire de $G$ (resp. $G^{\prime}$ ) dans $M$ (resp. $M^{\prime}$ ).

Si $P^{\prime}=P, l^{\prime}$ application $\xi \longrightarrow(\xi, \xi)$ de $P$ dans $P \otimes P$ permet d'identifier $P$ à un sous-fibré principal de $P \otimes P$ ( $G$ étant identifié à un sous-groupe de $G \times G$ par l'application diagonale). Si $\rho$ désigne une représentation linéaire de $G \times G$ dans un espace vectoriel $\mathbb{N}$, et si $\boldsymbol{\rho}_{0}$ désigne la restriction de $\mathcal{S}$ à $G$, les fibrés vectoriels $N, \mathcal{S}(G)[P \backsim P]$ et $f_{0}(G)[P]$ sont canoniquement isomorphes : on conviendre de les identifier. $\left.1^{\circ}\right)$ Produit tensoriel (1) de 2 fibrés vectoriels

Notons $Q \otimes Q^{\prime}$ la représentation linéaire de $G \times G^{\prime}$ dans $M \quad M^{\prime}$ définie par $\left(R \otimes R^{\prime}\right)\left(B, B^{\prime}\right)=R(B) \otimes R^{\prime}\left(B^{\prime}\right)$. On appellera "produit tensoriel" des fibrés $E=M_{Q(G)}[P]$ et $E^{\prime}=M^{\prime} R^{\prime}\left(G^{\prime}\right)$
$\left(\right.$ et $l^{\prime}$ on notera $\left.E \otimes E^{\prime}\right)$ le fibré vectoriel

$$
\left(M \otimes M^{\prime} M^{\prime}\right)\left(R \otimes R^{\prime}\right)(G \times G)\left[P \otimes P^{\prime}\right]
$$

Proposition I. A.1

(I) Pour tout point $x$ de $U$, il existe un isomorphisme canonique d'espaces vectoriels $\Phi_{x}: E_{x} \underset{\mathbb{R}}{\otimes} E^{\prime} \stackrel{\simeq}{\longrightarrow}\left(E \otimes E^{\prime}\right)_{x}$

(1) Il ne s'agit ici que de produits tensoriels internes 
(II) Il existe un isomorphisme canonique de $D(U)$-modules

$$
\begin{aligned}
& \Phi: \underset{m}{E} \underset{D(U)}{\otimes} E_{m}^{\prime} \stackrel{\simeq}{\longrightarrow} \underset{m}{E} E^{\prime} \text { défini par la condition : } \\
& \left(\oplus\left(s \otimes s^{\prime}\right)\right)(x)=\Phi_{x}\left(s(x) \otimes s^{\prime}(x)\right) \quad \forall s \in \underset{m}{E} \\
& \forall s^{\prime} \quad E^{\prime} \\
& \forall x \in U
\end{aligned}
$$

\section{Cas particulier $\mathrm{P}=\mathrm{P}^{\prime}$}

si $Q$ (resp. $Q^{\prime}$ ) est une représentation linéaire de $G$ dans $M$ (resp. $M^{\prime}$ ) et si $R_{0}$ désigne la restriction de $R \otimes \mathbb{R}^{\prime}$ à $G$ identifié à la diagonale de $G \times G$, on déduit des remarques préliminaires de ce paragraphe que

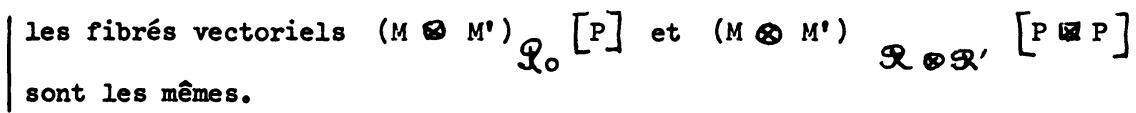
(On notera désormais encore $R \otimes R^{\prime}$ la représentation $R_{0}$ ).

$\left.2^{\circ}\right)$ Somne directe (1) (ou somme de Whitney) de 2 fibrés vectoriels Notons $R \oplus Q^{\prime}$ la représentation linéaire de $G \times G^{\prime}$ dans $M \oplus M^{\prime}$ définie par

$\left\langle\left(Q \oplus Q^{\prime}\right)\left(g, g^{\prime}\right), m+m^{\prime}\right\rangle=\langle Q(g), m\rangle+\left\langle Q^{\prime}\left(g^{\prime}\right), m^{\prime}\right\rangle$

$$
\forall\left(B, g^{\prime}\right) \in G \times G^{\prime}, \forall m+m^{\prime} \in M \oplus M^{\prime}
$$

On appellera "somme directe" ou "somme de Whitney" des fibrés vectoriels $E=M_{S(G)}[P]$ et $E^{\prime}=M_{R^{\prime}\left(G^{\prime}\right)}\left[P^{\prime}\right]$ (et $I^{\prime}$ on notera $E \oplus E^{\prime}$ ) le fibré

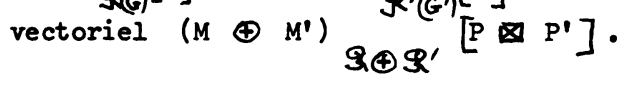

(1) Il ne s'agira que de sommes directes internes 


\section{Proposition I. A. 2}

(I) Pour tout point $x$ de $U$, il existe un isomorphisme canonique d'espaces vectoriels $r_{x}: E_{x} \oplus E_{x}^{\prime} \stackrel{\underline{n}}{\longrightarrow}\left(E \oplus E^{\prime}\right)_{x} \cdot$

(II) Il existe un isomorphisme cononique de $D(U)$ modules

$$
\begin{aligned}
& r: \underset{m}{E} \oplus \underset{E_{m}^{\prime}}{E^{\prime}} \simeq \underset{\text { E }}{\longrightarrow} E^{\prime} \text {, défini par la condition } \\
& \left(r\left(s+s^{\prime}\right)\right)_{(x)}=r_{x}\left(s(x)+s^{\prime}(x)\right) \quad \forall s \in \underset{\sim}{E} \quad \forall s^{\prime} \in \underset{m}{E^{\prime}} \quad \forall x \in U
\end{aligned}
$$

Si $P=P^{\prime}, Q_{I}$ désignant la restriction à la diagonale de $G$ G de la représentation $\mathscr{R} \oplus \mathfrak{Q}^{\prime}$, il résulte des remarques préliminaires que les fibrés $\left(M+M^{\prime}\right)_{Q_{I}}[P]$ et $\left(M \oplus M^{\prime}\right) \underset{R \oplus Q^{\prime}}{[P}\left[\begin{array}{lll}P & P\end{array}\right]$

sont les mêmes

(on notera encore $Q \oplus Q^{\prime}$ la restriction $Q_{1}$ ).

$\left.3^{\circ}\right)$ Puissances extérieures d'un fibré vectoriel

Notons $\stackrel{r}{\Lambda}$ la représentation linéaire de $G$ dans ${ }_{\Lambda}^{r} M$ défininie par

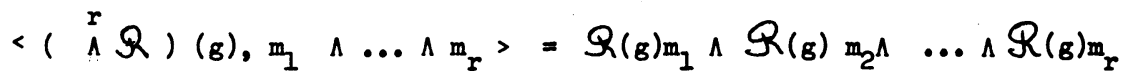

$$
\begin{aligned}
& \forall B \in G, \quad \forall m_{1} \wedge \ldots \wedge m_{r} \in \stackrel{r}{\Lambda}
\end{aligned}
$$

On appellera "puissance extérieure $\mathrm{r}^{\text {ième }}$ du fibré vectoriel

$E=M_{R}[P]$, le fibré vectoriel (noté $\stackrel{r}{\Lambda} E$ ) égal à

$\left(\begin{array}{llll}r & \Lambda & M\end{array}\right)_{r}[P]$. $\Lambda a$ 
Proposition I. A. 3

(I) Pour tout point $x$ de $U$, il existe un isomorphisme canonique

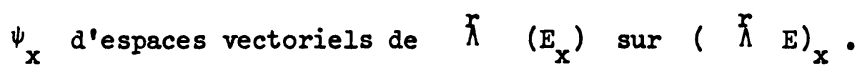

(II) Il existe un isomorphisme canonique $\psi$ de $D(U)$-modules de

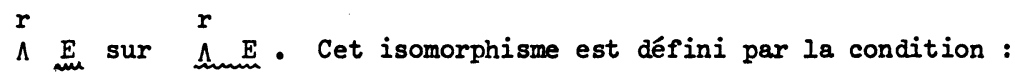

$$
\begin{aligned}
& \psi\left(s_{1} \wedge \ldots \wedge s_{r}\right) \quad(x)=\psi_{x}\left(s_{1}(x) \wedge \ldots \wedge s_{r}(x)\right) \\
& \forall s_{1}, \ldots, s_{r} \in \underset{m}{E}, \forall x \in U
\end{aligned}
$$

\section{$\left.4^{\circ}\right)$ Fibré dual d'un fibré vectoriel}

Si $R$ est une représentation linéaire de $G$ dans un espace vectoriel $M$, on notera $Q^{*}$ la représentation linéaire de $G$ dans le dual $M^{*}$ de $M$, définie par :

$$
Q^{*}(g)={ }^{t} Q\left(B^{-1}\right)
$$

$\left(<Q^{*}(g) u, m>=\left\langle u, Q\left(g^{-1}\right) m>\quad \forall g \in G, \forall m \in M, \forall u \in M^{*}\right)\right.$

On appellera "fibré dual" du fibré vectoriel $E=M_{Q}[P]$, le fibré vectoriel $M_{R}[P]$. On le notera $E^{*}$.

\section{Proposition I. A. 4}

(I) Pour tout point $x$ de $U$, il existe un isomorphisme canonique $\theta_{x}$ d'espaces vectoriels de $\left(E_{x}\right)^{*}$ sur $\left(E^{*}\right)_{x}$.

(II) Il existe un isomorphisme canonique $\theta$ de $D(U)$-modules de $(E)^{*}$ sur $\mathbb{E}_{m}^{*}$. Cet isomorphisme est défini par la condition :

$$
\begin{aligned}
\left(\theta(\alpha)(x)=\theta_{x}\left(a_{x}\right)\right. & \forall \alpha \in E^{*} \\
& \forall x \in U
\end{aligned}
$$




$$
\begin{aligned}
& \text { (où } \alpha_{x} \text { désigne l'élément de }\left(E_{x}\right)^{*} \text { défini par } \\
& \left\langle\alpha_{x}, m\right\rangle=\text { valeur en } x \text { de }\left\langle\alpha, \bar{m}>\forall m \in E_{x},\right. \\
& \bar{m} \text { désignant une section quelconque de } E \text { prenant en } x \text { la valeur } m \text { ). }
\end{aligned}
$$

$\left.5^{\circ}\right)$ Fibré vectoriel adjoint

si $Q$ est une représentation linéaire de $G$ dans $M, Q^{*} \otimes Q$ est une représentation linéaire de $G$ dans $E$ n d $M=M^{*} \otimes M$. On appellera "fibré adjoint" d'un fibré vectoriel donné $E=M \quad[P]$. le fibré ( $E$ ndM) $Q * \otimes[P]=E^{*} \otimes E \quad$ (on le notera $E$ ndE). Combinant les propositions 1 et 7 d'une part, 2 et 8 d'autre part, on voit que les espaces vectoriels (End $E)_{x}$ et End $\left(E_{x}\right)$ sont canoniquement isomorphes ainsi que les $D(U)$-modules End $D(U) \underset{\text { mm }}{E}$ et End $E$ • On vérifie aisément que :

$Q^{*} \otimes \mathscr{Q}$ est la représentation adjointe de $G$ dans End $M$.

\section{$\left.6^{\circ}\right)$ Les foncteurs modelage et section}

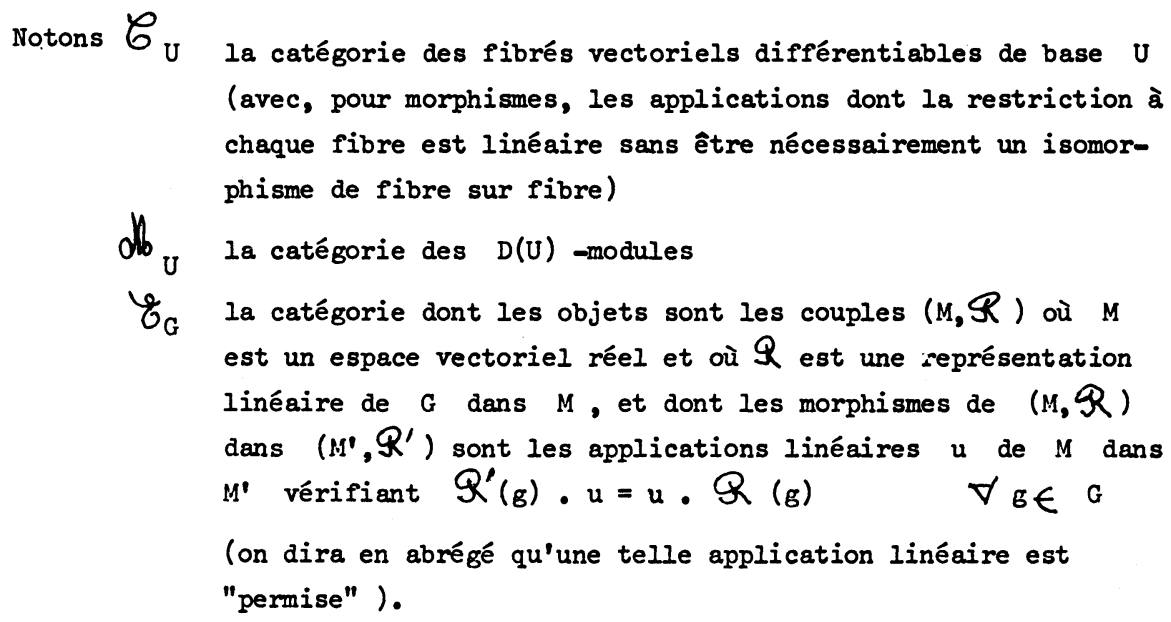


Soit $P$ un G-fibré principal différentiable de base $U$.

On vérifie aisément que le modelage sur $P$ définit un foncteur $\mu_{P}$ de la catégorie $\zeta_{G}$ dans la catégorie $\zeta_{U}$ :

$$
\left\{\begin{array}{l}
\mu_{P}(M, Q)=M_{Q}[P] \\
\mu_{P}(u) \text { est } I^{\prime} \text { application de } M_{Q}[P] \text { dans } M_{Q^{\prime}}^{\prime}[P] \text { définie par } \\
\mu_{P}(u)(q(m, \xi))=q^{\prime}(u(m), \xi) \\
\left(\text { ou } q \text { et } q^{\prime}\right. \text { désignent les applications canoniques } \\
\left.M \times P \longrightarrow M_{Q}[P] \text { et } M^{\prime} \times P \longrightarrow M_{Q^{\prime}}[P]\right)
\end{array}\right.
$$

Remarque : si u est une application linéaire permise injective, $\mu_{p}(u)$ est une injection. Plus généralement, $\mu_{P}$ est un foncteur exact.

$d_{U}$ en posant :

On définit aussi de façon évidente un foncteur $\sigma_{u}$ de $\zeta_{U}$ dans

$$
\begin{aligned}
& \sigma_{U}(E)=E \quad \text { pour tout fibré vectoriel } E \\
& \left(\sigma_{U}(\rho)\right) \sigma=\rho \circ \sigma \text { pour tout morphisme } P \text { de } E \text { dans } E^{\prime}
\end{aligned}
$$

Soit $Q$ une representation linéaire de $G$ dans $M$. On en déduit une représentation linéaire $\underline{Q}$ de $\underline{G}$ cans $M$. Soit ad la représentation linéaire adjointe de $G$ dans $\underline{G}$. L'application $\underline{R}$ de $\underline{G}$ dans End $M$ est une application linéaire permise de $(\underline{G}, a d)$ dans (End $M, \mathbb{R}^{*} \otimes \mathscr{R}$ ).

\section{Proposition I. A. 5}

On en déduit un homomorphisme $\mu_{P}(\underline{Q})$ de $\underline{G}_{\text {ad }}[P]$ dans $\operatorname{End}\left(M \mathbb{R}_{\mathbb{R}}[P]\right)$. si $R$ est fidèle, $\mu_{P}(\underline{R})$ est une injection. 


\section{$\left.7^{\circ}\right)$ Conventions}

Désormais, on conviendra toujours d'identifier

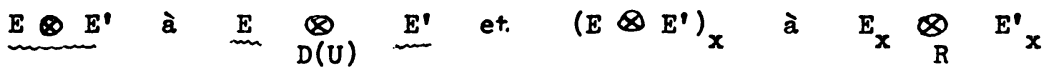

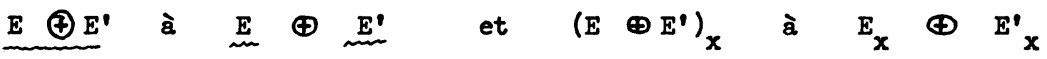

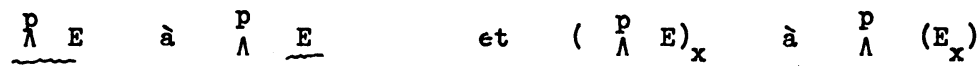

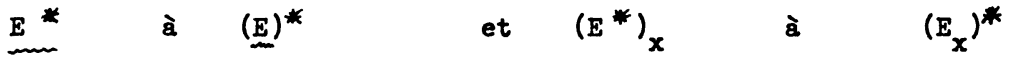

$\underline{G}$ ad $[P]$ à un sous-fibré vectoriel de End $E$, du moins lorsque $Q$ est fidèle.

B - Formes différentielles à valeurs dans un fibré vectoriel

$$
\text { Soient }\left\{\begin{array}{l}
P \text { un fibré principal (différentiable) de groupe } G \text { et de base } U, \\
R \text { une représentation linéaire de } G \text { dans un espace vectoriel } \\
\text { réel } M, \\
E=M_{Q}[P] \text { le fibré vectoriel associé, } \\
R(U) \text { le fibré principal des repères linéaires de } U \\
\text { (où l'un de ses sous-fibrés principaux) }
\end{array}\right.
$$

On appellera p-forme sur U à valeurs dans $\mathrm{E}$ un élément du $D(U)$ - module $R(T(U)) \underset{D(U)}{\mathbb{E}}, c^{\prime}$,est-à-dire une application $D(U)$-p-linéaire alternée $\alpha$ qui, à $p$ champs de vecteurs $x_{0}, \ldots, x_{p-1} \in \underset{\mathrm{Tm}}{T(U)}$, associe une section $\alpha\left(x_{0}, \ldots, X_{p-1}\right) \in \underset{m}{E}$.

On notera $\stackrel{\Lambda}{\Lambda}(U, E)$ le $D(U)$-module $\stackrel{\Lambda}{\Lambda} \underset{\text { mmm }}{(T(U))} \underset{D(U)}{\otimes} \underset{m}{E} \cdot$ 


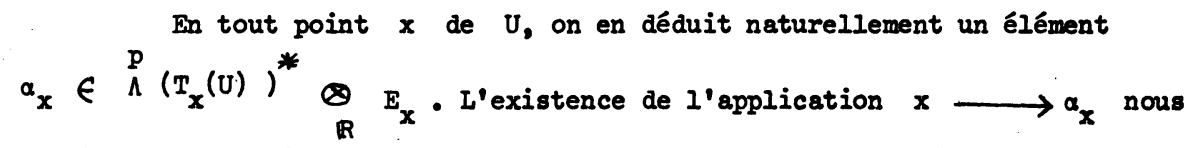
suggère de considérer $\alpha$ comme une section d'un certain fibré vectoriel. On déduit effectivement, des propositions 1 à 8 de I - A le

Théorème I. B. 1

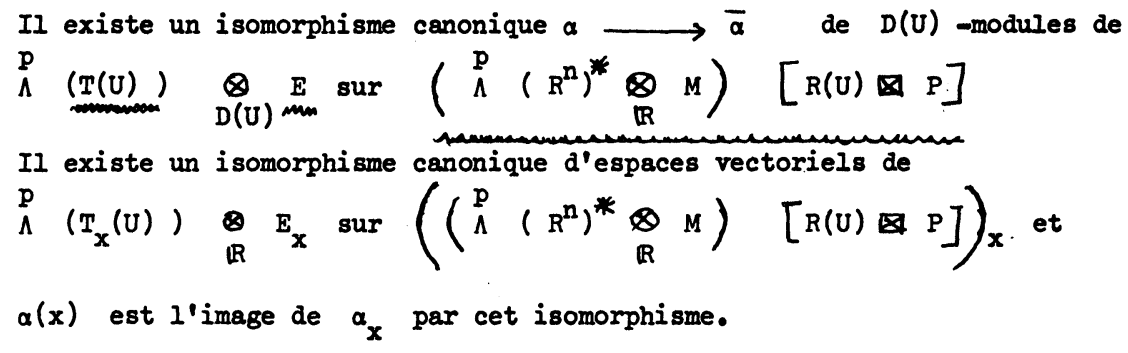

Correspondance entre formes sur $U$ à valeurs dans E, et formes tensorielles de type $R(G)$ sur $P$ à valeurs dans $M$ :

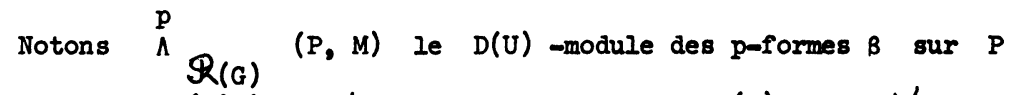

à valeurs dans $M$, vérifiant $\forall d_{0} \xi, \ldots, d_{p-1} \xi \in T_{\xi}(P)$ et $\forall B \in G$ :

$\left\{\begin{array}{l}\beta\left(d_{0} \xi, \ldots, d_{p-1} \xi\right)=0 \text { si l'un des vecteurs } d_{k} \xi \text { est vertical } \\ \beta\left(d_{0} \xi \cdot 8, \ldots, d_{p-1} \xi \cdot g\right)=Q\left(g^{-1}\right) \quad \beta\left(d_{0} \xi, \ldots, d_{p-1} \xi\right) .\end{array}\right.$

Théorème I. B. 2

\begin{tabular}{|l} 
Il existe un isomorphisme canonique $\alpha \stackrel{\lambda_{p}}{\longrightarrow} \tilde{a}$ de \\
$D(U)$-modules de $\stackrel{p}{\Lambda}(U, E)$ sur $\stackrel{p}{\Lambda}_{\mathscr{R}(G)}(P, M)$.
\end{tabular} 
Notons $\pi$ la projection $P \longrightarrow U$. Soit $\alpha \in \stackrel{p}{\Lambda}(U, E)$.

On définit une p-forme $\tilde{\alpha}$ sur $P$ à valeurs dans $M$, en posant :

$$
\tilde{\alpha}\left(d_{0}, \ldots, d_{p-1} \xi\right)=\xi^{-1} \alpha\left(\pi d_{0} \xi, \ldots, \pi d_{p-1} \xi\right)
$$

( $\xi$ est un isomorphisme $M \stackrel{\simeq}{\longrightarrow} E_{\pi \xi}$ ). Il est immédiat de vérifier que $\tilde{\alpha} \in \stackrel{p}{\Lambda}_{R(G)}(P, M)$ et que l'application $\lambda_{p}$
ainsi définie est un isomorphisme.

Dans le cas particulier $p=0$,

$\mid \begin{aligned} & \lambda_{0} \text { définit un isomorphisme de } D(U) \text {-modules de } \underset{\mathrm{m}}{\mathrm{E}} \text { sur l'espace } \\ & 0 \\ & { }^{\Lambda} R(G)(P, M) \text { des fonctions différentiables } \varphi \text { de } P \text { dans } M \text { vérifiant }\end{aligned}$

$$
\varphi(\xi g)=\left(\mathrm{B}^{-1}\right) \varphi(\xi) \quad \forall \xi \in P \quad \forall B \in G
$$

Combinant les théorèmes I. B. 1 et 2 , on en déduit le

\section{Corollaire I. B. 3}

$$
\begin{aligned}
& \text { Les } D(U) \text {-modules } \stackrel{p}{\Lambda}(U, E) \text { et } \stackrel{p}{\Lambda} R(G) \text { (P,M) sont canoniquement isomorphes à }
\end{aligned}
$$

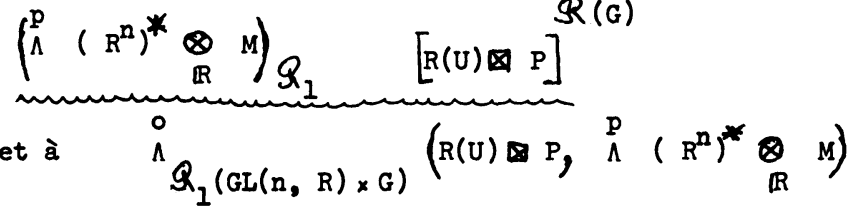

$$
\begin{aligned}
& \left(Q_{1} \text { désignant la représentation naturelle de } G I(n, R) \times G\right. \text { dans } \\
& \left.\begin{array}{lll}
p & \left.R^{n}\right)^{*} \underset{\mathbb{R}}{\otimes} & M
\end{array}\right) .
\end{aligned}
$$




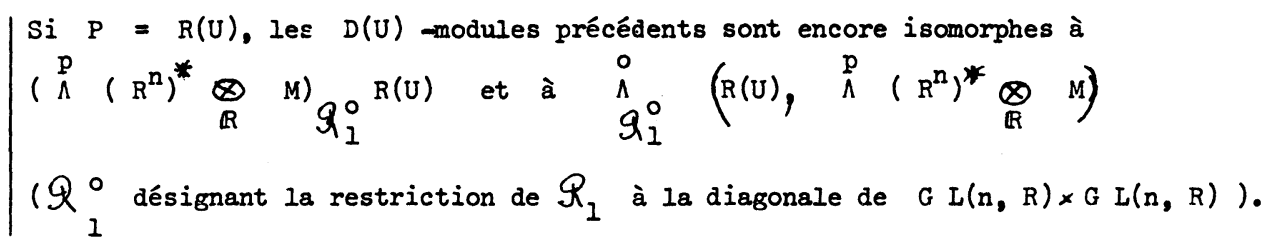

\section{Produits extérielirs}

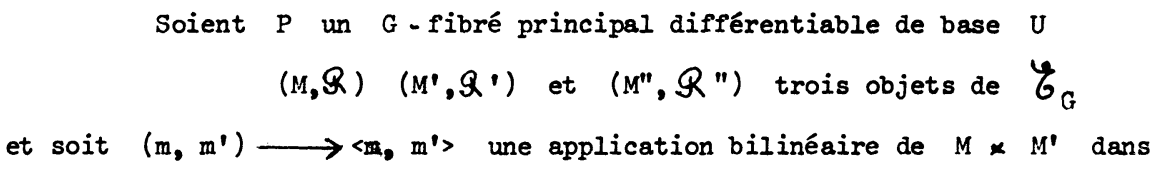

$$
\begin{aligned}
<(\mathrm{g}) \mathrm{m}, Q^{\prime}(\mathrm{g}) \mathrm{m}^{\prime}>=\mathbb{R}^{\prime \prime}(\mathrm{g})<\mathrm{m}, \mathrm{m}^{\prime}>\quad & \forall \mathrm{g} \in \mathrm{G} \quad \forall \mathrm{m} \in \mathrm{M} \\
& \forall \mathrm{m}^{\prime} \in \mathrm{M}^{\prime}
\end{aligned}
$$

Par application du foncteur composé $\sigma_{U} \cdot \mu_{P}$, on en déduit une application bilinéaire

$M_{Q}[P] \times M^{\prime} R^{\prime}[P] \longrightarrow M^{\prime \prime} Q^{\prime \prime}[P]$ notée < $>_{U} \cdot$

A l'aide de $<,>_{U}$, on définit un produit extérieur

$(\alpha, \beta) \longrightarrow \alpha \Lambda$ de $\stackrel{p}{\Lambda}\left(U, M_{R}[P]\right) \times \Lambda\left(U, M^{\prime} R^{\prime}[P]\right)$ dans

$\Lambda^{p+q}\left(U, M^{\prime \prime} Q^{\prime \prime}[P]\right)$. A l'aide de <, > on définit un produit extérieur

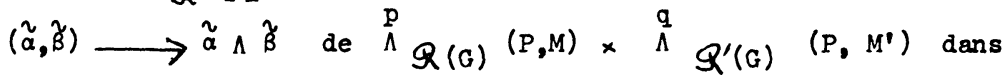

$\stackrel{p+q}{\Lambda} R^{\prime \prime}(G)\left(P, M^{\prime \prime}\right)$.

Théorème I. B. 4

$$
\begin{aligned}
& \left.\forall \alpha \in \stackrel{p}{\Lambda}^{\left(U, M_{Q}[P]\right), \quad \forall B \in \Lambda^{q}\left(U, M^{\prime}\right.} R^{\prime}[P]\right) \\
& \tilde{\alpha} \tilde{B}=\widetilde{\alpha \Lambda \beta}
\end{aligned}
$$


Remarque On a adopté la convention suivante pour les produits extérieurs :

$(\alpha \wedge \beta)\left(x_{1}, x_{2}, \ldots, x_{p+q}\right)=\left[(-1)_{\alpha}^{I}\left(x_{i_{1}}, \ldots, x_{i_{p}}\right) \beta\left(x_{i_{p+1}}, \ldots, x_{i_{p+q}}\right)\right.$

où la sommation $\sum$ est effectuée par rapport à toutes les permutations

$i_{1} \ldots i_{p} i_{p+1} \ldots i_{p+q}$ de $\{1, \ldots, p, p+1, \ldots q\}$

et où I désigne l'indice de la permutation. Il est à remarquer que nous ne faisons pas figurer $\frac{1}{(p+q) !}$ devant le signe $[$ et que nous ne nous restreienons pas aux permutations vérifiant

$i_{1}<i_{2}<\ldots<i_{p}$ et $i_{p+1}<i_{p+2}<\ldots<i_{p+q}$.

C - Connexions

Soit $P \stackrel{\pi}{\longrightarrow}$ U un G-fibré principal différentiable, $R$ une représentation linéaire de $G$ dans $M$, et $E$ le fibré vectoriel $M Q[P]$. Une connexion (ou connexion infinitésimale) sur $P$ est une 1 - forme $\omega$ sur $P$ à valeurs dans $\underline{G}$, vérifiant :

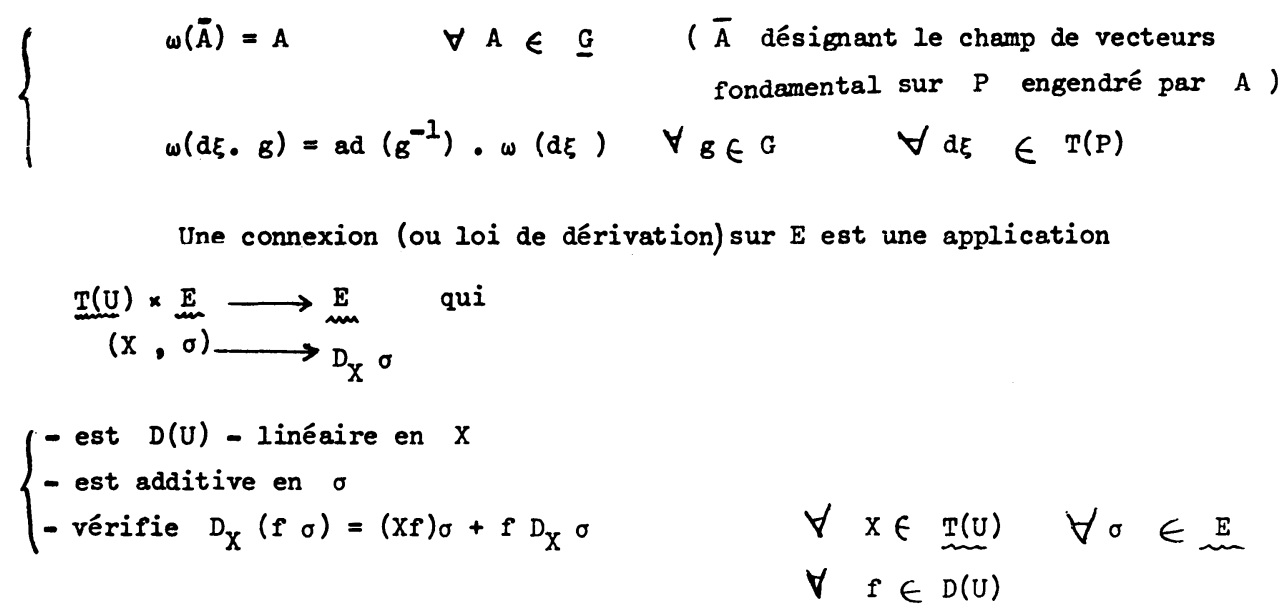


Nous allons rappeler brièvement comment les connexions sur $P$ et sur E se correspondent.

10) Transport parallèle sur E associé à une connexion sur $P$

Soit $\omega: T(P) \longrightarrow G$ une connexion sur $P$. Rappelons qu'un vecteur $d \xi \in T(P)$ est dit "horizontal" si $\omega(d \xi)=0$, qu'un chemin différentiable $\bar{\gamma}:[a, b] \longrightarrow P$ de $P$ est dit "horizontal" si tous ses vecteurs tangents sont horizontaux.

Soit $\gamma:[a, b] \longrightarrow U$ un chemin différentiable de $U$ d'extrémités $x_{a}$ et $x_{b}$. Pour tout $\xi_{a} \in P_{x_{a}}$, notons $J^{\gamma}\left(\xi_{a}\right)$ l'élément de $P_{x_{b}}$ obtenu comme extrémité de l'unique chemin horizontal $\bar{\gamma}$ de $P$ admettant $\xi_{a}$ comme origine et se projetant sur $\gamma: \xi_{a}\left(\right.$ resp. $J^{\gamma}\left(\xi_{a}\right)$ ) est un isomorphisme $M \stackrel{\simeq}{\longrightarrow} E_{x_{a}}$ (resp. $M \stackrel{\simeq}{\longrightarrow} E_{x_{b}}$ ), et $J^{\gamma}\left(\xi_{a}\right) \cdot\left(\xi_{a}\right)^{-1}$ est un isomorphisme de $E_{x_{a}}$ sur $E_{x_{b}}$. Du fait que $\omega(d \xi . g)=a d\left(g^{-1}\right) \cdot \omega(d \xi)$, on déduit par intégration le long de $\gamma$ que $J^{\gamma}\left(\xi_{a} \cdot g\right)=J^{\gamma}\left(\xi_{a}\right) \cdot g, \forall g \in G$.

Par conséquent, l'isomorphisme $J^{\gamma}\left(\xi_{a}\right) \cdot\left(\xi_{a}\right)^{-1}$ est indépendant de l'élément $\xi_{a}$ choisi dans $P_{x_{a}}$. On le notera désormais $J_{x_{a}}^{\gamma} x_{b}$ et on l'appellera le "transport parallèle de $E_{x_{a}}$ sur $E_{x_{b}}$ le long de $r$ ". Remarquons que $J_{x_{a}}^{\gamma} x_{b}$ est un isomorphisme, non seulement pour les structures d'espaces vectoriels de $E_{x_{a}}$ et $E_{x_{b}}$, mais encore pour les G-structures dont ces espaces sont munis : par exemple, si $G=S O(n)$ et si $Q$ est l'injection naturelle de $G$ dans $G I\left(R^{n}\right)$, chaque fibre de $E$ est munie naturellement $d^{\prime}$ une orientation et d'un produit scalaire, et $J_{x_{a}}^{\gamma} x_{b}$ conserve cette orientation et ce produit scalaire. 
$2^{\circ}$ ) Loi de dérivation sur E associée à une connexion sur P :

Une section $\sigma \in \underset{m}{E}$ sera dite "invariante" le long d'un chemin différentiable $t \stackrel{\gamma}{\longrightarrow} x_{t}$ de $U, s i$

$\sigma\left(x_{t}\right)=J_{x_{t},}^{\gamma} x_{t} \quad \sigma\left(x_{t},\right)$ quels que soient $t$ et $t^{\prime}$ appartenant à $I$ 'intervalle de définition de $r$.

La "variation moyenne" de $\sigma$ entre $x_{t}$ et $x_{t}$ ' sera donc mesurée par $\frac{1}{t^{\prime}-t}\left[\begin{array}{lll}J_{x_{t}}^{\gamma}, x_{t} & \sigma\left(x_{t},\right) & -\sigma\left(x_{t}\right)\end{array}\right]$ (élément de $E_{x_{t}}$ ).

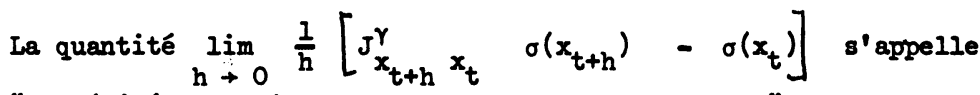

"la dérivée covariante de $\sigma$ en $x_{t}$, le long de $r$ ".

Théorème I. C. 1

(I) La dérivée covariante de $\sigma$ en $x_{t}$ le long de $\gamma$ ne dépend pas à proprement parler de $\gamma$, mais seulement du vecteur $x_{t}$ tangent à $\gamma$ en $x_{t}$; on la notera désormais $\mathrm{D}_{\mathrm{X}_{t}} \sigma$.

(II) L'application $x \longrightarrow D_{X(x)^{\sigma}}$ est une section différentiable de $E$, $\forall x \in \underset{\text { TI }}{T(U)} \quad \forall \sigma \in \underset{m}{\mathrm{E}}$.

(III) L'application $D:(X, \sigma) \longrightarrow D_{X^{\sigma}}$ ainsi définie est une loi de dérivation sur $E$.

(IV) Cette loi de dérivation $D$ est définie par

$\widetilde{D_{X^{\sigma}}}=x^{*} \cdot \tilde{\sigma} \quad \forall \sigma \in \underset{m}{E}, \quad \forall x \in \underset{m}{T(U)}$

( $\tilde{\sigma}$ et $D_{X} \sigma$ désignant les images de $\sigma$ et $D_{X} \sigma$ par l'isomorphisme $\lambda$ de $\underset{\mathrm{m}}{\mathrm{E}}$ sur ${ }^{\Lambda} \underset{R(G)}{ }(P, M)$, et $X^{*}$ désignant le relèvement horizontal de $X$ dans $P$ ). 
Il résulte en particulier de ce théorème, que si la représentation est fidèle, à deux connexions différentes sur $P$, sont alors associées deux connexions différentes sur $E$.

Il est faux que toute loi de dérivation sur $E$ provienne d'une connexion sur $P$ : par exemple, si $P$ est le fibré des repères orthonormés de $U$ relativement à une métrique riemanienne, et si $D$ est une connexion sur $T(U)$ (ou connexion linéaire), $D$ ne provient d'une connexion sur $P$ que si le transport parallèle de $D$ est une isométrie (on montre facilement qu'à toute loi de dérivation sur $E$, on associe un transport parallèle par intégration). Par contre, $D$ provient toujours d'une connexion dans le fibré de tous les repères de U. Plus généralement, on a le

Théorème I. C. 2

Si $G=G L(M)$ et $s i Q$ est la représentation cononique de $G$ dans $M$, toute loi de dérivation sur $E$ est l'image d'une connexion sur $P$ et d'une seule par l'application définie au théorème i. C. 1.

$\left.3^{\circ}\right)$ Courbure

Etant donnés $(P, \omega)$ et $E=M_{Q}[P]$ étant muni de la loi dérivation $D$ associée à $\omega$, on définit

- une 2 . forme $R \in{ }^{2} \Lambda$ (U, End $E$ ), en définissant pour tout couple $X, Y$ de $T(U)$ l'endomorphisme $R(X, Y)$ de $\underset{\mathrm{m}}{\mathrm{E}}$ par :$$
\left.R(X, Y)_{0}=D_{X} D_{Y^{\sigma}}-D_{Y} D_{X^{\sigma}}-D_{[X, Y}\right]^{\sigma}
$$

- une 2 . forme $\Omega \in \Lambda_{\text {ad } G}^{2}(P, G)$, en posant :

$\Omega\left(Z, Z^{\prime}\right)=d \omega\left(\right.$ Hor $Z$, Hor $\left.Z^{\prime}\right)$

(Hor $\mathrm{Z}$ et Hor $\mathrm{Z}^{\prime}$ désignant les parties horizontales de $\mathrm{Z}$ et $\mathrm{Z}$ ' relativement à $\omega)$. 
D'après I. B. $2, \Omega$ est l'image par $\lambda_{2}$ d'une 2 . forme

$$
\bar{\Omega} \in \stackrel{2}{\Lambda}(U, G \text { ad }[P])
$$

Théorème I. C. 3

$$
\begin{aligned}
& R=\mu_{P}(\underline{Q}) \cdot \bar{\Omega} \quad \text { où } \mu_{P}(\underline{\underline{Q}}) \text { désigne l'injection de } \underline{G} \text { ad }[P] \text { dans } \\
& \text { Fnd } E \text { définie à la proposition I. A. } 5 .
\end{aligned}
$$

D - Différentiation extérieure

$$
\text { Soient } \begin{cases}E=M & {[P] \text { un fibré vectoriel }} \\ \omega & \text { une connexion sur } P \\ D & \text { la loi de dérivation sur } E \text { associée à } \omega .\end{cases}
$$

A I'aide de $\omega$, on définit une application $R$ - linéaire

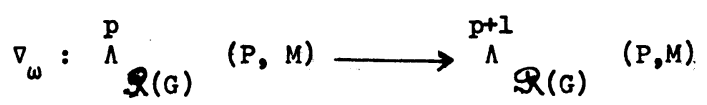

en posant :

$$
\left(\nabla_{\omega}, B\right)\left(z_{0}, \ldots, z_{p}\right)=d \beta\left(\text { Hor } z_{0}, \ldots, \text { Hor } z_{p}\right)
$$

où d est la différentiation extérieure habituelle Hor $z_{i}$ désigne la partie horizontale de $z_{i}$ relativement à $w$ •

A l'aide de $D$, on définit une application $R$ - linéaire

$$
d_{D}: \stackrel{p}{\Lambda}(U, E) \longrightarrow \Lambda^{p+l}(U, E)
$$


en posant :

$$
\begin{aligned}
&\left(\alpha_{p} \alpha\right)\left(x_{0}, \ldots, x_{p}\right)=\sum_{i=0}^{p}(-1)^{i} D_{x_{i}}\left(\alpha\left(x_{0}, \ldots, \hat{x}_{i}, \ldots, x_{p}\right)\right) \\
&+\sum_{i<j}(-1)^{i+j} \alpha\left(\left[x_{i}, x_{j}\right], x_{0}, \ldots, \hat{x}_{i}, \ldots, \hat{x}_{j}, \ldots, x_{p}\right) \\
& \forall x_{0}, \ldots, x_{p} \in \underset{m}{T(U)} .
\end{aligned}
$$

Théorème I. D. I

$$
\begin{aligned}
& d_{D} \text { et } \nabla_{\omega} \text { se correspondent par les isomorphismes } \lambda_{p} \text { de } \stackrel{p}{\Lambda}(U, E) \text { sur } \\
& \Lambda_{Q(G)}(P, M) \text { définis en I. B. } 2: \\
& \lambda_{p+1} \cdot d_{D}=\nabla_{\omega} \cdot \lambda_{p}
\end{aligned}
$$

Cas où $P$ est un "espace de repères" ; c'est-à-dire où le fibré tangent T(U) peut être obtenu comne modelé sur $\mathrm{P}$ :

$$
T(U)=\mathbb{R}_{\mathcal{R}_{0}}[\mathrm{P}] \text {. }
$$

Il existe une 1 - forme canonique id $\epsilon \stackrel{1}{\Lambda}(U, T(U))$, à savoir I'application $\mathrm{X} \longrightarrow \mathrm{X}$.

La 1 -forme $\theta=\lambda_{1}$ (id) $\in \Lambda^{1} Q_{0}(G)\left(P, \mathbb{R}^{n}\right)$ est appelée la

" forme fondementale " de l'espace de repères $P$.

Soit $\omega$ une connexion sur $P$, et $D$ la connexion sur $T(U)$ associée.

On appelle indistinctement "torsion" de la connexion considérée la 2 - porme $\mathbb{S}^{0}=d_{D}(i d) \in \Lambda(U, T(U))$ où la 2 - forme $\Sigma=\nabla_{\omega} \theta \in \Lambda^{2} \mathbb{R}_{0}(G)\left(P, R^{n}\right)$ - (D'après le théorème $I . D . I, \sum$ et $E$ se correspondent par $\lambda_{2}$ ). 
De la connexion sur $P$, on déduit une loi de dérivation (encore notée $D$ ) non seulement sur $T(U)$, mais encore sur tout fibré vectoriel

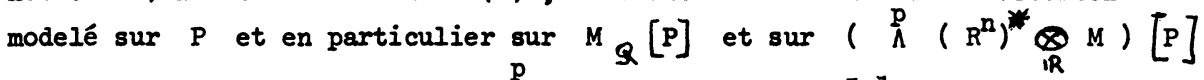
dont l'espace des sections est $\stackrel{p}{\Lambda}(U, E)$ où $E=M Q^{[P]}$. On vérifie aisément que si $\alpha \in \stackrel{p}{\Lambda}(U, E)$

$\left(D_{x} \alpha\right)\left(x_{0}, \ldots, x_{p-1}\right)=D_{x}\left[\alpha\left(x_{0}, \ldots, x_{p-1}\right)\right]-\sum_{i=0}^{p-1} \alpha\left(x_{0}, \ldots, D_{x} x_{i}, \ldots, x_{p-1}\right)$

De cette formule, de la définition de $a_{D} \alpha$, et de la formule

$\left[x_{i}, x_{j}\right]=-G_{i}\left(x_{i}, x_{j}\right)+D_{x_{i}} x_{j}-D_{x_{j}} x_{i} \quad$ qui définit la torsion, on déduit aisément la

\section{Proposition I.D.2}

$$
\begin{aligned}
& \left(d_{D} \alpha\right)\left(x_{0}, \ldots, x_{p}\right)=\sum_{i}(-1)^{i}\left(D_{x_{i}^{\alpha}}\right)\left(x_{0}, \ldots, \hat{x}_{i}, \ldots, x_{p}\right) \\
& +\sum_{i<j}(-1)^{i+j+1} \propto\left(\mathscr{G}\left(x_{i}, x_{j}\right), x_{0}, \ldots, \hat{x}_{i}, \ldots, \hat{x}_{j}, \ldots, x_{p}\right)
\end{aligned}
$$

Revenons au cas général où $P$ n'est plus nécessairement un espace de repères.

\section{Equations de structure}

Pour toute connexion $\omega$ sur $P$, on a le

Théorème I. D. 3 


$$
\begin{aligned}
& \Omega=d \omega+[\omega, \omega] \\
& \text { (où I'on a posé }[\omega, \omega]\left(z, Z^{\prime}\right)=\left[\omega(Z), \omega\left(Z^{\prime}\right)\right] \text { au sens du crochet } \\
& \text { dans G) } \\
& \nabla_{\omega}^{B}=d B+\omega \Lambda B \quad \forall B \in \Lambda_{R(G)}^{p}(P, M) \\
& \text { (où le produit extérieur } \omega \wedge \beta \text { est à comprendre au sens de } 1 a \\
& \text { forme bilinéaire canonique } \underline{G} \times M \longrightarrow M \text { déduite de la représen- } \\
& \text { tation } \underline{\Omega}: \underline{G} \longrightarrow \text { End } M \text { associée à la représentation : } \\
& \mathcal{Q}: \mathrm{G} \longrightarrow \mathrm{G} I(\mathrm{M}) \text { ). }
\end{aligned}
$$

Identités fondamentales (de Bianchi, et de Ricci)

Soit $E=M \&[P]$ muni d'une connexion $D$ associée à une connexion $\omega$ sur $P$. Notant encore $D$ la connexion sur End $E$ associée à $\omega$, on a l'identité de Bianchi :

Théorème I. D. 4

$d_{D} R=0 \quad$ où $R \in \Lambda_{\Lambda}^{2}$ (U, End $E$ ) est la 2-forme de courbure

D'après I. C. 3 et I. D. I, il revient au même d'écrire cette identité :

$$
\nabla_{\omega} \Omega=0
$$

Soit $\sigma \in \underset{m}{E}: c^{\prime}$ est une o-forme $\in \stackrel{\circ}{\Lambda}(U, E)$ dont la différentielle extérieure $d_{D} \sigma n^{\prime}$ est autre que la l-forme $x \stackrel{D \sigma}{\longrightarrow} D_{X} \sigma$ qui apparient à $\Lambda(U, E)$. D'après la définition même de la forme de courbure, $R(X, Y) \sigma=\left(d_{D} D \sigma\right)(X, Y)$ donc $R(X, Y) \sigma=\left(d^{2} \sigma\right)(X, Y)$. Plus généralement, on a l'identité de Ricci : 
Théorème I. D. 5

$d_{D^{\alpha}}^{2}=R_{\Lambda} \alpha \quad \forall \quad \alpha \in \stackrel{p}{\Lambda}_{(U, E)}$

le produit extérieur de $R \in \Lambda_{\Lambda}^{2}$ (U, End $E$ ) par a étant à comprendre au sens de la forme bilinéaire canonique

End $\mathbf{E} \times \mathbf{E} \longrightarrow \underset{\mathbf{E}}{\longrightarrow}$

D'après I. B. 4, I.C. 3 et I. D. 1 , il revient au même d'écrire cette identité

$$
\nabla_{\omega}^{2} B=\Omega_{\Lambda} \beta \quad \forall B \in \stackrel{p}{R(G)}_{(P, M)}
$$

où le produit extérieur est à prendre au sens de la forme bilinéaire canonique $\mathrm{G} \times \mathrm{M} \longrightarrow \mathrm{M}$, déduite de la représentation $\mathrm{Q}: \underline{G} \longrightarrow$ End $M$ associée à la représentation $Q]$. 


\author{
A - Plongements d'espaces fibrés \\ formes de plongement \\ connexions induites \\ B - Connexions sur un produit fibré \\ C - Premiers exemples \\ 10) G-connexions \\ $\left.2^{\circ}\right)$ Connexions de Cartan \\ $\left.3^{\circ}\right)$ Espaces de Minkowski \\ $4^{\circ}$ ) Espaces homogènes réductifs
}


A - Plongements d'espaces fibrés - Formes de plongement - Connexions induites

Soient $G$ un groupe de Lie, et $H$ un sous-groupe de Lie.

Puisque la représentation adjointe ad de $H$ dans $G$ conserve le sous-espace $\underline{H}$ de $G$, elle induit une représentation linéaire ad de H dans $G / H$. définie par $\underline{a d}(\mathrm{~h}) \cdot \pi=\pi$. ad $(\mathrm{h}) \quad \forall \mathrm{h} \in \mathrm{H}$ (où $\pi$ désigne la projection canonique de $G$ sur $\underline{G} / \underline{H}$ ).

Si l'espace homogène $G / H$ est réductif et si $\bar{d}$ désigne un supplémentaire de E dans $G$ tel que ad(H) dl $\subset d b$, la restriction de $\pi$ à clb est un isomorphisme permis de $(d b, a d)$ sur $(\underline{G} / \underline{H}, \underline{a d})$ dans la catégorie $\xi_{H}$ des espaces vectoriels sur lesquels H opère linéairement.

On dira qu'un morphisme de variétés $U \longrightarrow V$ "vérifie la condition R. D " (initiales de : rétraction par déformations) si l'une des deux hypothèses suivantes est satisfaite :

- ou bien $U=V$ et $P$ est l'identité

- ou bien $U$ et $V$ sont dénombrables à l'infini et il existe un morphisme $v \stackrel{r}{\longrightarrow} U$ tel que $f$. $r$ soit homotope à (id) $v$ et $r_{0} f=(i d)_{u}$.

Soient $P \stackrel{P}{\longrightarrow} U$ (resp. $Q \stackrel{Q}{\longrightarrow}$ V) un H fibré principal (resp. G).

On appellera "plongement de $P$ dans $Q$ " un couple $(f, p)$ d'applications ou $f$ est un morphisme de $U$ dans $V$ et $\rho$ un plongement de $P$ dans l'image réciproque $Q f$ de $Q$ par $P$, réalisant $P$ comme sous-fibré principal de Qf .

Donnons nous une connexion $\omega$ sur $Q$, et un plongement $(f, \rho)$ de $P$ dans $Q$. Notons $l$ application canonique de $Q f$ dans $Q$. On appelle "forme de plongement" de $P$ dans $Q$ relativement à $(f, \rho, \omega)$ la l-forme $P l$ sur $P$ à valeurs dans $G / \underline{H}$, image réciproque de $\pi \cdot \omega$ par $\tilde{f} \cdot \rho$. 
On vérifie que :

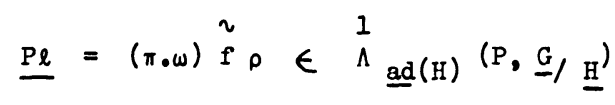

Supposons désormais $G_{/ H}$ réductif et soit db un supplémentaire de $\underline{H}$ dans $G$ invariant par ad(H). On appellera encore forme de plongement la l-forme $P_{l}$ sur $\rho$ à valeurs dans do , égale à $\omega_{d_{0}} \cdot \tilde{e}_{\rho}$. (Il est clair que $\mathrm{Pl}$ et $\mathrm{Pl}$ se correspondent par $\mathrm{l}^{\prime}$ isomorphisme permis $\pi$ de $d b$ sur $G / \underline{H}$ ). Notons d'autre part $\omega^{\prime}$ la l-forme sur $P$ à valeurs dans $H$, égale $\grave{a} \omega_{H} \cdot \tilde{f} \rho \cdot$ On a alors le

Théorème II. A. I

(I) la l-forme $\omega^{\prime}=\omega_{\underline{H}} \cdot \tilde{f} \rho$ est une forme de connexion sur $P$

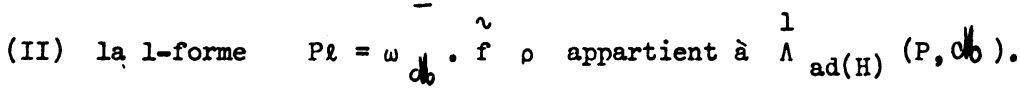

$$
\text { Puisque } \rho(\xi h)=\rho(\xi) . h \quad \forall \xi \in P, \quad \forall h \in H,
$$

on peut affirmer :

- d'une part que l'image par $\rho$ du champ de vecteurs fondamental $\bar{A}$ sur $P$ engendré par l'élément $A$ de $\underline{H}$, est la restriction à $\rho(P)$ du champ de vecteurs fondamental $\overline{\bar{A}}$ sur $Q \bar{f}$ engendré par $A$,

- d'autre part que $\rho(d \xi \circ h)=\rho(d \xi) . h \quad \forall d \xi \in T_{\xi}(P) \quad \forall h \in H$.

On en déduit, compte tenu de ce que $\omega \tilde{f}$ est une connexionsur Qf :

- d'une part que $\omega^{\prime}(\bar{A})=A$ et $P \ell(\bar{A})=0$

- d'autre part que $\left(\omega^{\prime}+P \ell\right)(d \xi \cdot h)=$ ad $(h)\left(\omega^{\prime}+P \ell\right)(d \xi)$. 
Mais puisque ad(H) $\mathrm{H}<\underline{\mathrm{H}}$ et $\mathrm{ad}(\mathrm{H}) \mathrm{cll} \mathrm{C} \mathrm{C}$ b , la représentation adjointe de $H$ dans $G$ commute avec les projections de $G$ sur $\underline{H}$ et $c$ (parallèlement à do et $\underline{H}$ ). On en déduit :

$$
\omega^{\prime}(d \xi, h)=\operatorname{ad}\left(\hbar^{l}\right) \cdot \omega^{\prime}(d \xi) \text { et } \quad P l(d \xi, h)=\operatorname{ad}\left(\hbar^{l}\right) \cdot P l(d \xi) .
$$

La connexion $\omega^{\prime}$ sera dite "induite" par $(\omega,(f, p), d b)$.

\section{Formules induites par les équations de structure}

Soient $\omega^{\prime}$ et $\mathrm{Pl}$ la connexion et la forme de plongement induites sur $P$ par $(\omega,(f, p), d b)$.

\section{Proposition II. A. 3}

(I) $\Omega \quad \sim_{\rho}^{\prime}=\Omega^{\prime}+\nabla_{\omega^{\prime}}, \mathrm{Pl}+[\mathrm{Pl}, \mathrm{Pl}]$

( $\Omega$ et $\Omega^{\prime}$ désignant respectivement les courbures de $\omega$ et $\omega^{\prime}$ ).

(II)
$\alpha \in \stackrel{p}{\Lambda}_{R(G)}(Q, M)$,
$\alpha \tilde{f} \rho \stackrel{p}{\Lambda}_{Q^{\prime}(H)}(P, M)$ et

$\left(\nabla_{\omega} \alpha\right) \tilde{f} \rho=\nabla_{\omega},(\alpha \tilde{f} \rho)+P l_{\Lambda} \alpha \tilde{f} \rho$

( $Q$ désignant une représentation linéaire de $G$ dans $M_{0}$ désine la représentation de $H$ dans $M$ obtenue par restriction de $Q$ à $H$, le produit extérieur $P l \Lambda$ ấp étant à comprendre au sens de la forme bilinéaire canonique $G \times M \quad M$ définie par la représentation $\underline{Q}$ : G $\longrightarrow$ End M)

De l'équation de structure $\quad \Omega=d \omega+[\omega, \omega]$, on déduit : 


$$
\begin{aligned}
& \Omega \tilde{f}_{\rho}=d(\omega \tilde{f} \rho)+[\omega \tilde{f} \rho, \omega \tilde{f} \rho] \\
& =\mathrm{d}\left(\omega^{\prime}+\mathrm{Pl}\right)+\left[\omega^{\prime}+\mathrm{Pl}, \omega^{\prime}+\mathrm{Pl}\right] \\
& =\left(\mathrm{d} \omega^{\prime}+\left[\omega^{\prime}, \omega^{\prime}\right]\right)+\left(\mathrm{dPl}+\omega_{\Lambda}^{\prime} \mathrm{Pl}\right)+[\mathrm{Pl}, \mathrm{Pl}] \\
& \text { Mais } \mathrm{d} \omega^{\prime}+\left[\omega^{\prime}, \omega^{\prime}\right]=\Omega^{\prime} \text { et d Pl+ } \omega^{\prime}{ }_{\Lambda} \mathrm{Pl}=\nabla_{\omega} \mathrm{Pl} \text {, d'où (I). } \\
& \text { De l'équation se structure } \nabla_{\omega} \alpha=d \alpha+\omega_{\Lambda} \alpha \text {, on déduit : }
\end{aligned}
$$

$$
\begin{aligned}
\left(\nabla_{\omega} \alpha\right) \tilde{f}_{\rho} & =d(\alpha \tilde{f} \rho)+\left(\omega \tilde{f}_{\rho}\right) \Lambda(\alpha \tilde{f} \rho) \\
& =d(\alpha \tilde{f} \rho)+\omega^{\prime} \Lambda(\alpha \tilde{f} \rho)+P l \Lambda \alpha \tilde{f}_{\rho}
\end{aligned}
$$

Mais $d(\alpha \tilde{f} \rho)+\omega^{\prime} \Lambda(\alpha \tilde{f} \rho)=\nabla_{\omega^{\prime}}\left(\alpha \tilde{\tilde{f}_{\rho}}\right) \quad \mathrm{d}^{\prime}$ où (II)

Le fait que $\alpha \tilde{f}_{\rho} \in \stackrel{p}{\Lambda}_{Q^{\prime}(H)}(P, M)$ lorsque $\alpha \in \stackrel{p}{\Lambda} Q(G)$ (P, M) est absolument immédiat).

On dira que $(f, p)$ est un plongement "affine" (resp."développable") de $P$ dans $(Q, \omega)$ si $P l=0$ (resp. $\left.\Omega \tilde{f} \rho=\Omega^{\prime}\right)$. Un plongement est donc développable si et seulement si

$$
\nabla_{\omega}, \mathrm{Pl}+[\mathrm{Pl}, \mathrm{Pl}]=0
$$

Il en est en particulier ainsi d'un plongement affine.

\section{Equations de GAUSS-CODAZZI}

Si l'on se donne un plongement $(f, p)$ de $P$ dans $Q$ une forme de connexion $\omega^{\prime}$ sur $P$ une 1-forme $\mathrm{Pl} \in \Lambda^{1}$ ad $\mathrm{H}(\mathrm{P}, \mathrm{db})$

on peut se demander $8:$ il existe une connexion $\omega$ sur $Q$ induisant $\omega^{\prime}$ et $\mathrm{Pl}$. La réponse est fournie par la 


\section{Proposition II. A. 4}

(I) Si $f$ vérifie la condition R.D., il existe une connexion $\omega$ sur $Q$ telle que $\omega^{\prime}$ et $P l$ soient induites par $(\omega,(f, \rho), d b)$. En outre, si $U=V$ et si $f=(i d)_{u}$, il n'existe qu'une seule telle connexion $\omega$.

(II) $\mathrm{Si} f$ vérifie R.D. , une condition nécessaire et suffisante pour qu'il existe sur $Q$ une connexion $\omega$ à courbure nulle induisant $\omega^{\prime}$ et $\mathrm{Pl}$ est que les données $\omega^{\prime}$ et $\mathrm{Pl}$ vérifient l'équation : $\Omega^{\prime}+\nabla_{\omega}, \mathrm{Pl}+[\mathrm{Pl}, \mathrm{Pl}]=0 \quad$ (équation de GAUSS-CODAZZI).

Il existe en effet sur $Q f$ une connexion $\omega_{1}$ et une seule telle que $\omega_{1} \rho=\omega^{\prime}+P l$. Soit $r$ un morphisme $V \longrightarrow U$ tel que $f \cdot r \sim(\text { id })_{v}$ et $r . f=(i d)_{u}$. La forme $\omega_{I} \cdot r$ est une connexion sur Qfr, induisant $\omega_{1}$ sur $Q f=(Q f r) f$.

Il existe un isomorphisme $\tilde{\varphi}$ de $Q$ fr sur $Q$, tel que sa projection $\varphi: v \longrightarrow V$ vérifie $\varphi$. $f=f$. Soit $\omega$ l'image réciproque de $\omega_{1} \cdot r$ par $\tilde{\varphi}^{-1}$; $\omega f$ est égal à $\omega_{1}$. Il est clair que $\omega$ est une connexion sur $Q$ induisant $\omega^{\prime}$ et $\mathrm{Pl} \operatorname{par}((f, p), d b)$.

$$
\text { La condition } \Omega^{\prime}+\nabla_{\omega^{\prime}} \mathrm{Pl}+[\mathrm{Pl}, \mathrm{Pl}]=0 \text { est évidement néces- }
$$
saire pour qu'il existe une telle connexion $\omega$ à courbure nulle, ceci en vertu de la formule (I) de II. A. 3. Réciproquement, si cette condition est vérifiée, la courbure $\Omega_{1}$ de $\omega_{1}$ est nulle : en effet $\Omega_{1}\left(\rho d_{1} \xi, \rho d_{2} \xi\right)=0$ $\forall d_{1} \xi, d_{2} \xi \in T_{\xi}(P)$ d'après cette même formule $(I)$ de II. A. 3 ; comme les vecteurs verticaux de $T_{\rho}(\xi)(Q f)$ et les vecteurs tangents à $\rho(P)$ engendrent tout $T_{\rho}(\xi)(Q f)$, on a $\Omega_{I}(X, Y)=0 \quad \forall X, Y \in T_{p}(\xi) \quad(Q f), \quad \forall \xi \in P$. Comme $\Omega_{1}$ est de type ad $G$, on en déduit $\Omega_{1}=0$. Puisque $\omega$ est l'image réciproque de $\omega_{1}$ par $r \cdot \varphi^{-1}, \Omega$ aussi est nulle. 
Exemple (qui jouera un rôle important au chapitre IV)

Prenons

$$
\begin{aligned}
& \text { pour } P \text { le fibre principal } G \longrightarrow G / H, \\
& \text { pour } \omega^{\prime} \text { la forme de connexion } \omega^{\prime}\left(d_{g}\right)=\left(g^{-1} d_{g}\right)_{H} \\
& \text { pour } P l \text { la l-forme } P l\left(d_{g}\right)=\left(g^{-1} d_{g}\right)_{d}
\end{aligned}
$$

La condition $\quad \Omega^{\prime}+\nabla_{\omega^{\prime}} \mathrm{Pl}+[\mathrm{Pl}, \mathrm{Pl}]=0$ est satisfaite.

Les champs de vecteurs horizontaux en $P$ sont engendrés par les champs de vecteurs invariants à gauche $\vec{M}$ sur $G$ définis par les éléments $M$ de $d_{b}^{\prime}$ ( dै $($ G) - (ce. $[14]$ p. ).

Il suffit donc de vérifier que

$$
\begin{aligned}
& \left(\Omega^{\prime}+\nabla_{\omega^{\prime}} \mathrm{Pl}+[\mathrm{Pl}, \mathrm{Pl}]\right)(\overline{\mathrm{M}}, \overline{\mathrm{N}})=0 \quad \forall \mathrm{M}_{,} N \in d \mathrm{~d} . \\
& \text { Or } \quad \Omega^{\prime}(\bar{M}, \bar{N})=-[M, N]_{\underline{H}} \text {, } \\
& \left(\nabla_{\omega}, \mathrm{Pl}\right)(\mathrm{M}, \mathrm{N})=\bar{M}_{\ell} \operatorname{Pl}(\overline{\mathrm{N}})-\overline{\mathrm{N}} \cdot \operatorname{Pl}(\overline{\mathrm{M}})-\mathrm{Pl}(\overline{\mathrm{M}}, \overline{\mathrm{N}}) \\
& =-\operatorname{Pl}(\bar{M}, \bar{N})=-[M, N]_{d b} \text {. } \\
& {[\mathrm{Pl}, \mathrm{Pl}](\overline{\mathrm{M}}, \overline{\mathrm{N}})=[\mathrm{Pl}(\overline{\mathrm{M}}), \mathrm{Pl}(\overline{\mathrm{N}})]}
\end{aligned}
$$

d'où l'égalité cherchée.

Plus simplement, $\Omega^{\prime}+\nabla_{\omega^{\prime}} \mathrm{Pl}+[\mathrm{Pl}, \mathrm{Pl}]$ est égal à $\mathrm{d}\left(\omega^{\prime}+\mathrm{Pl}\right)+\left[\omega^{\prime}+\mathrm{Pl}, \omega^{\prime}+\mathrm{Pl}\right]$, et l'équation de GAUSS-CODAZZI $\mathrm{n}^{\prime}$ est autre ici, que l'équation de MAURER-CARTAN du groupe G .

B - Connexions sur un produit fibré

Soient $P^{\prime} \stackrel{p^{\prime}}{\longrightarrow} U$ (resp. $P^{\prime \prime} \stackrel{p^{\prime \prime}}{\longrightarrow} U$ ) un fibré principal de groupe structural G' (resp. G") et $\omega^{\prime}$ (resp. $\omega^{\prime \prime}$ ) une connexion sur $P^{\prime}$ (resp. $\left.P^{\prime \prime}\right)$. 


$$
\text { Si }\left(\xi^{\prime}, \xi^{\prime \prime}\right) \in P_{x}^{\prime}{ }^{\prime \prime}{ }_{x}=\left(P^{\prime} P^{\prime \prime}\right)_{x}, I^{\prime} \text { espace }
$$

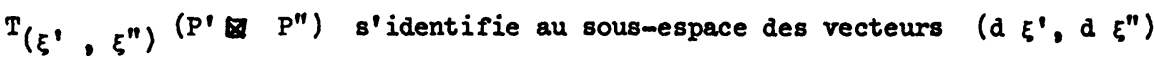
de $T_{\left(\xi^{\prime}, \xi^{\prime \prime}\right)}\left(P^{\prime} \times P^{\prime \prime}\right)=T_{\xi^{\prime}}\left(P^{\prime}\right) \oplus T_{\xi^{\prime \prime}}\left(P^{\prime \prime}\right)$ tel que $p^{\prime}\left(d \xi^{\prime}\right)=p^{\prime \prime}\left(d \xi^{\prime \prime}\right)$. Identifiant alors $G^{\prime} \times G^{\prime \prime}$ avec $G^{\prime} \oplus G^{\prime \prime}$, on définit une 1-forme $w$ sur $P^{\prime} \otimes P^{\prime \prime}$ à valeurs dans $G^{\prime} \times G^{\prime \prime}$, en posant :

$\omega\left(d \xi^{\prime}, d \xi^{\prime \prime}\right)=\omega^{\prime}\left(d \xi^{\prime}\right)+\omega^{\prime \prime}\left(d \xi^{\prime \prime}\right) \quad \forall\left(d \xi^{\prime}, d \xi^{\prime \prime}\right) \in T_{\left(\xi^{\prime}, \xi^{\prime \prime}\right)}\left(P^{\prime} \& P^{\prime \prime}\right)$

Il est clair que $\omega$, ainsi définie, est une connexion sur le $G^{\prime} \times G^{\prime \prime}$ - fibré principal $P^{\prime} \otimes P^{\prime \prime}$, que $l^{\prime}$ on appellera la "connexion produit" (ou plus simplement le produit) de $\omega^{\prime}$ et $\omega^{\prime \prime}$.

On se propose, réciproquement, de montrer la

Proposition II. B. 1

(I) Tout fibré principal $Q \longrightarrow U$ de groupe structural $G=G^{\prime} \times G^{\prime \prime}$ est canoniquement isomorphe au produit fibré du G'-fibré principal $Q / G^{\prime \prime} \longrightarrow U$ et du $G^{\prime \prime}$.fibré principal $Q / G^{\prime} \longrightarrow U$.

(II) Pour toute connexion $\omega$ sur $Q$, il existe une et une seule connexion $\omega^{\prime}$ sur $Q / G^{\prime \prime}$ ainsi qu'une et une seule connexion $\omega^{\prime \prime}$ sur $Q / G^{\prime}$, telles que $\omega s^{\prime}$ identifie au produit des connexions $\omega^{\prime}$ et $\omega^{\prime \prime}$ par l'isomorphisme de la partie (I).

La lère partie du théorème est évidente. Identifions désormais $Q$ et $Q / G^{\prime \prime} \quad Q / G^{\prime}$. Pour démontrer la 2ème partie, remarquons que la projection sur $\underline{G}^{\prime}$ de $\omega\left(d \xi^{\prime}, d \xi^{\prime \prime}\right)$ ne dépend que de $d \xi^{\prime}$, et que cette projection doit nécessairement être égale à $\omega^{\prime}\left(d \xi^{\prime}\right)$ si $\omega^{\prime}$ existe. On définit ainsi une forme $\omega^{\prime}$ sur $P^{\prime}$ à valeurs dans $G^{\prime}$, qui est en fait une forme de connexion. On définit $\omega^{\prime \prime}$ de façon analogue; il est alors clair que $\omega$ est bien le produit de $\omega^{\prime}$ et $\omega^{\prime \prime}$. 
42

lère Partie : Quelques propriétés des connexions induites

Soit $E^{\prime}=M^{\prime}\left[P^{\prime}\right]$ (resp. $E^{\prime \prime}=M^{\prime \prime}\left[P^{n}\right]$ ) un fibre vectoriel muni de la connexion $D^{\prime}$ (resp. $D^{\prime \prime}$ ) associée à une connexion $\omega^{\prime}$ sur $P^{\prime}$ (resp. $\omega^{\prime \prime}$ sur $P^{\prime \prime}$ ). Soit $\omega$ la connexion sur $P^{\prime} P^{\prime \prime}$ égale au produit de $\omega^{\prime}$ et $\omega^{\prime \prime}$.

- Ia loi de dérivation $D$, associée ¿̀ $\omega$, sur le fibré vectoriel $E^{\prime} \odot E^{\prime \prime}=\left(M^{\prime} \bigotimes_{\mathbb{R}} M^{\prime \prime}\right)\left[P^{\prime} \otimes P^{\prime \prime}\right]$ est définie par :

$$
\begin{aligned}
& D_{\mathrm{X}}\left(\sigma^{\prime} \otimes \sigma^{\prime \prime}\right)=\left(D_{\mathrm{X}}^{\prime} \sigma^{\prime} \otimes \sigma^{\prime \prime}\right)+\left(\sigma^{\prime} \otimes \mathrm{D}_{\mathrm{X}}^{\prime \prime} \sigma^{\prime \prime}\right) \\
& \forall \sigma^{\prime} \in \underset{\mathrm{m}}{\mathrm{E}^{\prime}}, \quad \forall \sigma^{\prime \prime} \in \underset{\mathrm{m}}{\mathrm{E}^{\prime \prime}}, \forall \mathrm{X} \in \underset{\mathrm{T}(U)}{\mathrm{T}(U)} .
\end{aligned}
$$

- la loi de dérivation $D^{\perp}$ associée à $\omega$ sur $\left(E^{\prime} \oplus E^{\prime \prime}=\left(M^{\prime} \oplus M^{\prime \prime}\right)\left[P^{\prime} \otimes P^{\prime \prime}\right]\right.$ est definie par :

$$
\begin{aligned}
& D_{X}^{1}\left(\sigma^{\prime}+\sigma^{\prime \prime}\right)=D_{X}^{\prime} \sigma^{\prime}+D_{X}^{\prime \prime} \sigma^{\prime \prime} \\
& \forall \sigma^{\prime} \in \underset{m}{E^{\prime}}, \forall \sigma^{\prime \prime} \in \underset{m}{E^{\prime \prime}}, \forall x \in \underset{\text { Tim }}{T(U)}
\end{aligned}
$$

En effet, le relèvement whorizontal $X^{*}$ de $X$ dans $T\left(P^{\prime} \backsim P^{\prime \prime}\right)$ est égal à $\left(X^{\prime *}, X^{\prime \prime *}\right)$, où $X^{\prime *}$ (resp. $X^{\prime \prime *}$ ) est le relèvement $\omega$ '-horizontal (resp. $\omega^{\prime \prime-h o r i z o n t a l) ~ d e ~} X$ dans $T\left(P^{\prime}\right)$ (resp. $T\left(P^{\prime \prime}\right)$ ).

$$
\begin{aligned}
& \text { Notant } \tilde{\sigma} \text { l'image d'une section } \sigma \text { par } \lambda_{0} \text {, on } a \text { : }
\end{aligned}
$$

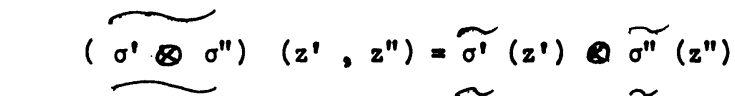

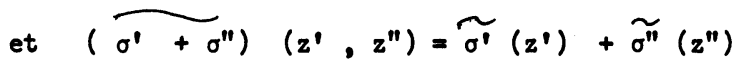

Donc

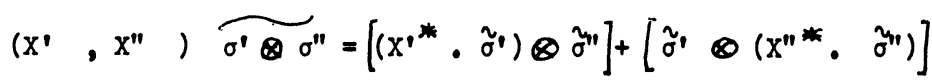

$$
\begin{aligned}
& \text { et }\left(X^{\prime}, X^{\prime \prime}\right) \widetilde{\sigma^{\prime}+\sigma^{\prime \prime}}=\left(X^{\prime *}, \tilde{\sigma}^{\prime}\right)+\left(X^{\prime \prime} \cdot \tilde{\sigma}^{\prime \prime}\right) \text {. }
\end{aligned}
$$


Il suffit donc d'appliquer I. C. I pour en déduire les formules annoncées.

C - Premiers exemples

\section{0) G-connexions}

Soit $E \longrightarrow U$ un fibré vectoriel de fibre type $\mathbb{R}^{n}$, et notons $P_{E}$ le $G L(n, R)$-fibré principal qui lui est associé.

Soit $G$ un sous-groupe de Lie de $G L(n, \mathbb{R})$ : une G-struc-. ture ${ }^{(1)}$ sur $E$ est un sous-fibré principal $P$ de $P_{E}$ admettant $G$ comme groupe structural.

Soit $M$ un espace vectoriel réel, et $\mathbb{R}$ une représentation linéaire de $G L(n, R)$ dans $M$. Suivant BERNARD $[1]$, on dira que la G-structure $P\left(P C P_{E}\right)$ est définie par un tenseur

$t \in \AA_{R(G L(n, R))}\left(P_{E}, M\right), s^{\prime} i l$ existe un élément $u$ de $M$ tel que $P$ soit l'espace des éléments $\xi$ de $P_{E}$ vérifiant $t(\xi)=u$ (le groupe $G$ étant alors l'ensemble des éléments $g$ de $G L(n, R)$ tels que $Q(g) . u=u)$. Réciproquement, on sait d'après [I] que la donnée d'un tenseur $t \in \stackrel{\Lambda}{Q R(G L(n, R)}^{\left(P_{E}, M\right)}$ et d'un élément $u$ de $M$ définissent une G-structure sur E, pourvr que les deux conditions suivantes soient réalisées :

(1) Le terme de G-structure est employé dans un sens un peu plus général que dans $[1]$ : on ne suppose pas nécessairement que $E=T(U)$. 
(I) $\forall x \in U, \quad \exists \in P_{E}$ tel que $t(\xi)=u$

(II) l'application $t: P_{E} \longrightarrow M_{u} C M$ est différentiable, non seulement quand on la considère comme prenant ses valeurs dans $M$, mais encore quand on $1 a$ considère comme à valeurs dans la classe d'intransitivité $M_{u}$ de $u$ (c'est-à-dire l'orbite de $u$ lorsque $G L(n, \mathbb{R})$ opère $\operatorname{sur}(M)$.

[1a signification de la deuxième condition est la suivante : $M_{u}$ est égal ¿̀ l'espace homogène $\frac{G L(n R)}{G}$; or les G-structures correspondent biuniroquement aux sections différentiables du fibré modelé $\left(\frac{G L(n, R)}{G}\right)_{G L(n, R)}\left[P_{E}\right] \rightarrow U, c^{\prime}$ est-à-dire aux fonctions différentiables $t$ de $P_{E}$ dans $\frac{G L(n, R)}{G}$ vérifiant $t(\xi s)=s^{-1} \cdot t(\xi) \quad \forall \xi \in P_{E}$ $\forall B \in G I(n, R)$ : le cas ơ la G-structure est définie par un tenseur appartenant à $\stackrel{\circ}{Q(G L(n, R)}\left(P_{E}, M\right)$ correspond simplement à la donnée d'un plongement différentiable de $\frac{G L(n, R)}{G}$ dans $M$, tel que la restriction de $Q_{\text {à }} G L(n, \mathbb{R})$ s'identifie à l'opération naturelle à gauche de $G L(n, R)$ sur cet espace homogène $]$.

Soit $P$ une G-structure sur $E$; l'injection $Z$ de $P$ dans $P E$ perwet de definir un plongement $\left(\eta,(i d)_{u}\right)$ de $P$ dans $P_{E}$ : soit $P l=\pi(\omega \eta) \in \Lambda_{\text {ad } G}^{l}\left(P, \frac{\text { End } \mathbb{R}^{n}}{G}\right.$ ) la forme de plongement induite par 
$\left(\eta,(i d)_{u}\right)$ et une connexion w sur $P_{E}$ (où $\pi$ désigne la projection canonique de End $\mathbb{R}^{n}$ sur $\frac{\text { End } \mathbb{R}^{n}}{G}$ ). Nous dirons, suivant [2] , que $\omega$ est une "G-connexion" sur $\mathrm{E}$, si $\mathrm{Pl}=0$.

Supposons la G-structure définie par un tenseur

$t \in \stackrel{\Lambda}{R(G L(n, R)})_{\left(P_{E}, M\right)}\left(P\right.$ est $l$ 'ensemble des éléments $\xi$ de $P_{E}$ vérifiant $t(\xi)=u)$. Dans [1], BERNARD a montré qu'une condition nécessaire et suffisante pour que $\omega$ soit une G-connexion est que $t$ ait une dérivée convariante nulle par rapport à $\omega: \nabla_{\omega} t=0$. Nous allons montrer, plus généralement, que $\mathrm{Pl}$ détermine entièrement la dérivée covariante $\nabla_{\omega} t \in \Lambda_{Q(G L(n, \mathbb{R}))}^{1}\left(P_{E}, M\right):$ Notons $(A, m) \longrightarrow\langle A, m\rangle$ I'application bilinéaire canonique End $\mathbb{R}^{\mathrm{n}} \times \mathrm{M} \longrightarrow \mathrm{M}$ définie par $\mathbb{R}$ : Puisque $\mathcal{R}(\mathrm{B}) \mathrm{u}=\mathrm{u} \quad \forall \mathrm{B} \in \mathrm{G}$, $\langle A, u\rangle=0 \quad \forall A \in G$ et par conséquent $\left\langle A^{I}\right.$, $u>$ ne dépend que de $\pi A^{I}$ $\left(A^{I} \in\right.$ End $\left.\mathbb{R}^{n}\right)$ : on notera encore $<\pi A^{l}$, u> cet élément. On a alors la :

Proposition II. C. I

$$
\left(\nabla_{\omega} t\right) \eta=\langle\underline{P l}, u\rangle
$$

où $\langle\underline{P l}$, u> est la l-forme sur $P$ à valeurs dans $M$, définie par $\langle\underline{P l}, u\rangle(d \xi)=\langle\underline{P l}(d \xi), u\rangle$

$$
\begin{array}{ll}
\text { En effet } & \nabla_{\omega} t=d t+\langle\omega, t\rangle \\
\text { Donc } & \left.\left(\nabla_{\omega} t\right) \tau=d(t \imath)+\langle\omega \eta, \text { t }\rangle\right\rangle .
\end{array}
$$


Mais $t q$ est la fonction constante égale à $u$, et $d(t q)=0$.

Par conséquent : $\left(\nabla_{\omega} t\right) \eta=\langle\omega \eta, u\rangle$

$=\left\langle\pi \omega_{2}, u\right\rangle$

$=\langle\underline{P l}, u\rangle, d^{\prime}$ où la proposition.

Dans le cas où l'espace homogène $\frac{G L(n, R)}{G}$ est réductif, soit db un supplémentaire de $G$ dans End $\left(\mathbb{R}^{\mathrm{n}}\right)$ stable par ad(G). Notant $\omega^{\prime}$ la projection $\omega_{G}$ de $\omega$ sur $G$ parallèlement à $d b$, il résulte de II. A. 1.que $\omega^{\top}$ est une connexion sur $P$. Appliquant II. A. 4, on voit qu'il existe sur $P_{E}$ une G-connexion $\widehat{\omega^{\prime}}$ et une seule induisant $\omega^{\prime}$ : Cette connexion $\widehat{\omega^{\prime}}$ sera appelée "la G-connexion induite par $\omega$ " (qui n'est évidemment égale à $\omega$ que dans le seul cas où $\omega$ est déjà une G-connexiond.

\section{$2^{\circ}$ ) Connexions de CARTAN}

$\begin{array}{ll}\text { Soient } \quad Q & \longrightarrow U \text { un G-fibré principal } \\ & \text { H un sous-groupe de Lie de G } \\ & \sigma \text { une section différentiable du fibré } \\ & \text { modelé }(G / H)[Q] \longrightarrow U \text {. }\end{array}$

Cette section $\sigma$ s'identifie canoniquement à la donnée d'un sous-fibré principal $P$ de $Q$ admettant $H$ comme groupe structural, et $I$ 'on conviendra d'identifier $(G / H)[Q]$ et $(G / H)[P]$ qui sont canoniquement isomorphes en tant que variétés différentiables fibrées en espaces homogènes.

On définit un fibré vectoriel $\left(G_{/ H}\right)_{\text {Qd }}[P] \longrightarrow U$ en faisant opérer linéairement $H$ sur $G / H$ par la représentation ad définie au début du paragraphe A de ce chapitre. Géométriquement, $\left(\left(\left(_{G} / H\right)[P]\right) \times\right.$ s'identifie 
canoniquement à $T_{\sigma(x)}((G / H)[P])_{x}$ quand on identifie $G_{/ H}$ à $T_{e_{0}}(G / H)$, e。 désignant la projection sur $G / H$ de l'élément neutre de $G$.

Suivant EHRESMANN [4], on appellera "soudure" de $U$ sur le fibré en espaces homogènes $G / H[P]$ tout isomorphisme de fibrés vectoriels de $T(U)$ sur $\underline{G}\left[\underline{H}\left[\right.\right.$ P] (se projetant sur $(i d)_{u}$ ).

La donnée d'une soudure (qui implique évidemment que $U$ et $G / H$ ont même dimension) est équivalente à la donnée d'une structure d'espace de repères sur $P$, c'est-à-dire d'une l-forme $\underline{P l} \in \Lambda_{\text {ad } H}(P, \underline{G} / \underline{H})$ telle que $\underline{P l}(d \xi) \neq 0$ pour tout vecteur $d \xi$ tangent à $\bar{P}$ non vertical (cf. $[1]$ ).

Notant toujours $\pi$ la projection $G \longrightarrow G / H$, ceci justifie la définition suivante :

Suivant [4], on appellera "connexion de CARTAN" une connexion $\omega:$ $T(Q) \longrightarrow G$ sur $Q$, telle que la forme de plongement $\underline{P l}=\pi(\omega \mid P)$ de $P$ dans $(Q, \omega)$ soit une forme d'espace de repères (c'est-à-dire vérifie $\underline{P l}(\dot{d} \xi) \neq 0$ pour tout vecteur $d \xi$ tangent à $P$ non vertical).

Cette forme $\underline{P_{l}}$ sera appelée ici la "forme de soudure" relative à la connexion de CARTAN $\omega$.

Supposons désormais $G / H$ réductif, et soit $d$ b un supplémentaire de $\underline{H}$ dans $\underline{G}$, stable par ad(H). Comme corollaire de II. A. I et II. A. 4, on obtient la

Proposition II. C. 2

L'application $\omega \longrightarrow\left((\omega \mid P)_{\underline{H}},(\omega \mid P)_{\phi}\right)$ est une correspondance biunivoque de l'ensemble des connexions de CARTAN sur $Q$ sur l'ensemble 
des couples formés d'une connexion $(\omega \mid P)_{H}=\omega^{\prime}$ sur $P$ et d'une forme
de soudure $(\omega \mid P)_{d b}=P l \in \Lambda_{\text {ad } H}(P, C b)$. (ob et $G / \underline{H}$ étant maintenant identifiés par $\pi / c / b)$.

Munissant $P$ d'une strucutre d'espace de repères à l'aide de $\mathrm{Pl}, \omega^{\prime}$ est en particulier une connexion linéaire sur $U$, dont la torsion $\sum$ est égale à $\nabla_{\omega}, \mathrm{Pl}$. Notant alors $\Omega$ et $\Omega^{\prime}$ les courbures de $\omega$ et $\omega^{\prime}$, la formule (I) de II. A. 3 devient ici

$$
\Omega \mid \mathrm{P}=\Omega^{\prime}+[+[\mathrm{Pl}, \mathrm{Pl}]
$$

Par projections sur $\underline{H}$ et sur $c_{b}$, on obtient :

$$
\begin{aligned}
& (\Omega \mid \mathrm{P})_{\underline{H}}=\Omega^{\prime}+[\mathrm{Pl}, \mathrm{Pl}]_{\underline{H}} \\
& (\Omega \mid \mathrm{P})_{d b}=\left[+[\mathrm{Pl}, \mathrm{Pl}]_{d b}\right.
\end{aligned}
$$

Interprétation géométrique

La connexion $\omega$ sur $Q$ permet de définir une notion de transport parallèle le long d'un chemin de $U$ pour tout fibré associé à $Q$ (même non vectoriel), et en particulier pour le fibré $G / H[Q] \longrightarrow U$. Soit $x_{0}$ un point de $U$ et $Y$ un lacet "infinitésimal" en $x_{0}$; le transport parallèle le long de $r$ est un "déplacement infinitésimal" de la fibre en $x_{0}$ (qui est un espace homogène) sur elle-même : Ce déplacement infinitésimal est mesuré par un élément $A \in G$ : un repère de $(G / H[Q])_{x_{0}}$ est défini par 
- un point $y \in(G / H[Q])_{x_{0}}$

- un repère linéaire de l'espace $q$ tangent $T_{y}\left[\begin{array}{lll}(G / H & Q & )_{x_{0}}\end{array}\right]$

- la composante $\mathrm{A}_{d b}$, entièrement définie par $\left[+[\mathrm{Pl}, \mathrm{Pl}]_{\mathrm{db}}\right.$, représente la variation de $\mathrm{y}$

- la composante $\mathrm{A}_{\underline{H}}$, entièrement définie par $\Omega+[\mathrm{Pl}, \mathrm{Pl}]_{\underline{H}}$, représente la rotation du repère linéaire une fois $y$ fixé.

Dans le cas des connexions affines, $G$ est le groupe affine
$A(n, \mathbb{R})$ de $R^{n}, H$ est le groupe linéaire $G L(n, \mathbb{R}) \cdot$
Puisque $A(n, R)$ est le produit semi-direct de $G L(n, \mathbb{R})$ par le
groupe abélien $R^{n}$, db est alors isomorphe à $R^{n}$ et $[d b, d b]=0$. On en déduit $[\mathrm{Pe}, \mathrm{Pe}]=0$ et

$$
\begin{aligned}
& (\Omega \mid \mathrm{P})_{\mathrm{GL}(\mathrm{n}, \mathrm{R})}=\Omega^{\prime} \\
& (\Omega \mid \mathrm{P})_{\mathrm{R}^{\mathrm{n}}}=\sum
\end{aligned}
$$

d'où une interprétation géométrique de la torsion comme la composante translation du déplacement affine de l'espace tangent sur lui-même obtenu par transport parallèle le long d'un cycle infiniment petit (cf. LICHNEROWICZ [16] p. 93 et E. CARTAN [2] p. 180).

\section{$\left.3^{\circ}\right)$ Espaces de MINKOWSKI}

L'algèbre de Lie $O(n)$ est la sous-algèbre de Lie de End $\left(\mathbb{R}^{n}\right)$, formée des matrices antisymétriques. Prenons pour db l'espace des matrices symétriques de End $\left(\mathbb{R}^{\mathfrak{n}}\right)$ : on vérifie aisément que 


$$
\left\{\begin{array}{l}
\text { End }\left(\mathbf{R}^{n}\right)=\underline{o(n)}+d b, \\
\text { ad }(o(n)) d b c d b, \\
{[d b, d b] \subset \underline{o(n)}}
\end{array}\right.
$$

Soit $P C P_{E}$ une $O(n)$-structure sur un fibré vectoriel trivial $E=\mathbb{R}^{\mathrm{n}} \times \mathrm{U} \longrightarrow U$ : il revient au même de se donner différentiablement une métrique euclidienne sur $\mathbb{R}^{\mathfrak{n}}$ pour tout point $x$ de $U$. Le fibré principal $P_{E}=G I(n, R) \times U$ admet une connexion canonique à courbure nulle :

$$
\left.\omega(d B, d x)=\vec{B}^{-1} d B \quad \forall(d g, d x) \in T(g x) G L I(n, R) \times U\right) .
$$

Soit $\mathcal{Z}$ I'injection canonique de $P$ dans $P_{E}$ •

La connexion $\omega^{\prime}$ induite sur $P$ par $\left(\omega,\left(2, i d_{u}\right), d b\right)$ sera appelée la connexion canonique de $P$.

$$
\text { La l-forme de plongement } \mathrm{Pl} \in \stackrel{1}{\Lambda}_{O(n)}\left(\mathrm{P}, d_{b}\right) \text { mesure l'écart }
$$
qui existe entre $P$ et le fibré trivial $O(n) \times U \rightarrow U:$ la nullité de $\mathrm{Pl}$ équiraut en effet au fait que les métriques euclidiennes sur $R^{n}$, associées par $P$ à chaque point $x$ de $U$, sont toutes les mêmes.

La formule (1) de II. A. 3 devient ici :

$$
\begin{aligned}
& \Omega^{\prime}+[\mathrm{Pl}, \mathrm{Pl}]=0 \\
& \nabla_{\omega_{l}, \mathrm{Pl}}=0
\end{aligned}
$$

\section{Cas particulier :}

Prenons pour $U$ la variété des directions orientées de $R^{n}$ (quotient de $\mathrm{R}^{\mathrm{n}}-\{0\}$ par la relation d'équivalence :

$$
x \sim \lambda x \quad \forall \lambda>0)
$$


Pour tout $x \in \mathbb{R}^{\mathrm{n}}-\{0\}$, notons

$j_{x}: R^{n} \stackrel{\simeq}{\longrightarrow} T_{x}\left(\mathbb{R}^{n}-\{0\},\right)$ I'isomorphisme canonique d'espaces vectoriels associé à la structure affine canonique de $\mathbb{R}^{n}$. Pour tout vecteur $X \in \mathbb{R}^{n}$, notons $j \times$ le champ de vecteurs tangent $\grave{a} R^{n}-\{0\}$ dont la valeur en un point $x$ de $\mathbb{R}^{n}-\{0\}$ est $j_{X}(X) \quad$ (si $X, Y \in \mathbb{R}^{n}$, on a $[j X, j Y]=0$ ). Soit $J$ le champ de vecteurs tangent à $\mathbb{R}^{n}-\{0\}$ tel que $J(x)=j_{x}(x)$. Une fonction différentiable $f: \mathbb{R}^{\mathrm{n}}-\{0\} \longrightarrow \mathbb{R}$ est dite "positivement homogène de degré $\alpha$ " si

$$
f(\lambda x)=\lambda^{\alpha} f(x) \quad \forall x \in \mathbb{R}^{\mathbb{n}}-\{0\} \quad \forall \lambda>0 .
$$

Une telle fonction vérifie l'identité d'Euler

$$
J \cdot P=\alpha f
$$

et, pour tout vecteur $x \in \mathbb{R}^{n}$, la fonction $j x . f$ est positivement homogène de degré $\alpha-1$.

Soit $L: \mathbb{R}^{n}-\{0\} \longrightarrow \mathbb{R}^{+}$une fonction différentiable à valeurs positives, positivement homogène de degré 1 . A tout point $x$ de $\mathbb{R}^{\mathrm{n}}-\{0\}$, on associe une forme bilinéaire symétrique $g_{x}$ sur $\mathbb{R}^{\mathfrak{n}}$ en posant;

$$
g_{x}(X, Y)=j_{x}(X) \cdot j Y \cdot \frac{1}{2} L^{2}
$$

(il est clair que cette fonction de $\left(\mathbb{R}^{n}\right)^{2}$ dans $R$ est linéaire en $X$; elle est d'autre part symétrique en $X$ et $Y$, donc linéaire en $Y$ puisque $[j X, j Y]=0)$. Puisque $\frac{1}{2} L^{2}$ est positivement homogène de degré 2 , $j X \cdot j Y \cdot \frac{1}{2} L^{2}$ est positivement homogène de degré 0 , ce qui implique :

$$
g_{x}=g_{\lambda x} \quad \forall x \in \mathbb{R}^{n}-\{0\} \quad \forall \lambda>0
$$


La métrique $g_{x}$ ne dépend donc que de la direction $\delta:$ on 12 notera désormais $B_{\delta}$.

Suivant GUIJWAMAIN $[7]$, on dira que $l^{\prime}$ on munit $\mathbb{A}^{n}$ d'une structure "d'espace de MINKOWSKI" si l'on s'est donné une fonction différentiable $L: \mathbb{R}^{n}-\{0\} \longrightarrow R^{+}$, positivement homogène de degré 1 , telle que les métriques $B_{\delta}$ associées soient toutes définies positives.

Une telle structure est donc en particulier une $O(n)$-structure $P$ sur le fibré vectoriel trivial $R^{n} \times U \longrightarrow U$. Il existe donc une connexion canonique sur $P$, et la nullité de $P l$ équivaut au fait que l'espace de MINKOWSKI est en fait un espace euclidien ( $L$ associant à tout vecteur $X$ de $R^{n}$ sa norme euclidienne $\left.\|X\|\right)$ ).

\section{$\left.4^{\circ}\right)$ Espaces homogènes réductifs}

Soit $G / H$ un espace homogène réductif, et $\mathrm{d}$ b un supplémentaire de $\underline{H}$ dans G vérifiant $a d(H)$ db CClb - Soit $P_{0}$ le H-fibré principal

$G \stackrel{P}{\longrightarrow G / H}$. On peut considérer $P_{0}$ comme sous-fibre principal du G-fibré trivial $G \times G / H$ : l'injection $\eta$ de $P_{O}$ dans $G \times G / H$ étant celle qui associe $(B, G H)$ à $G$ où $B$ H désigne la projection $p(B)$ de $8 \operatorname{sur} G / H \cdot$

Soit $\tilde{\omega}$ la connexion canonique à courbure nulle sur $G \times G / H \rightarrow G / H$ définie par $\tilde{\omega} \quad(d g, d x)=\bar{B}^{-1} d g \quad \forall d g \in T_{g}(G), \forall d x \in T_{x}(G / H)$. A l'aide du plongement $\left(2,(i d)_{G / H}\right), \omega$ induit une connexion $\omega_{0}$ sur $P_{0}$ et une 1-forme $P_{0} \in \Lambda_{\text {ad }}^{\frac{1}{\Lambda}}\left(P_{0}, d b\right)$ vérifiant :

$$
\begin{aligned}
\omega_{0}\left(\dot{d}_{g}\right) & =\left(\vec{G}^{-1} d g\right)_{H} \\
P_{0}(d g) & =\left(\vec{B}^{-1} d g\right)_{d b}
\end{aligned}
$$


Cette connexion $\omega_{0}$ est la connexion canonique de l'espace homogène réductif. Puisque $\mathrm{Pl}_{0}\left(\mathrm{dg}_{g}\right)=0$ implique $p\left(d_{g}\right)=0, \mathrm{Pl}_{0}$ est une forme d'espace de repères et $\omega_{0}$ est une connexion linéaire sur $G / H$ (invariante à gauche).

Cette construction de $\omega_{0}$ explique pourquoi l'équation de GAUSS-CODAZZI est vérifiée (cf. II. A. Exemple final) . 


\author{
A - Projections d'une loi de dérivation \\ B - Transcription en termes de fibrés principaux \\ C - Connexions canoniques sur les variétés de \\ Stiefel généralisées \\ D - Géométrie des sous-variétés
}


A - Projections d'une loi de dérivation

Soit $\mathrm{E} \longrightarrow \mathrm{U}$ un fibré vectoriel, somme de WHITNEY de deux sous-fibrés vectoriels $E^{\prime} \longrightarrow U$ et $E^{\prime \prime} \longrightarrow U$. On a alors $\underset{m}{E}=\underset{m}{E^{\prime}} \oplus \underset{m}{E^{n}}$; on identifiera $\underset{m}{E^{\prime}}$ (resp. $\left.\underset{m}{E^{\prime \prime}}\right)$ à un sous $D(U)$-module de $\underset{m}{E}$, et on notera $p^{\prime}\left(\right.$ resp. $\left.p^{n}\right)$ la projection de $\underset{\mathrm{m}}{\mathrm{E}} \operatorname{sur} \underset{\mathrm{m}}{\mathrm{E}^{\prime}}\left(\mathrm{resp} . \underset{\mathrm{m}}{E^{n}}\right.$ ) paral-

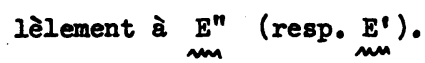

Nous avons déjà vu ( $f$ in du paragraphe II. B) que si $D^{\prime}$ et $D^{\prime \prime}$ sont des lois de dérivation sur $E^{\prime}$ et $E^{\prime \prime}$, on définit une loi de dérivation $D$ sur $E$ en posant :

$$
D_{X^{\sigma}}=D_{X X}^{\prime}\left(p^{\prime} \sigma\right)+D_{X}^{n}{ }^{\left(p^{\prime \prime} \sigma\right)} \quad \forall X \in \underset{T}{T(U)}, \quad \forall \sigma \in \underset{\operatorname{ma}}{E} .
$$

On a, réciproquement, la

\section{Proposition III. A. I}

Pour toute loi de dérivation D sur E,

(I) I'application $D^{\prime}:\left(X, \sigma^{\prime}\right) \longrightarrow D^{\prime}{ }_{X} \sigma^{\prime}$, définie par $D^{\prime}{ }_{X} \sigma^{\prime}=p^{\prime}\left(D_{X} \sigma^{\prime}\right)$, est une loi de dérivation sur E' [resp. 1'application $\left.D^{\prime \prime}:\left(X, \sigma^{\prime \prime}\right) \longrightarrow D_{X}^{\prime \prime} \sigma^{\prime \prime}\right)=p^{\prime \prime}\left(D_{X} \sigma^{\prime \prime}\right)$ est une loi de dérivation $\left.\operatorname{sur} E^{\prime \prime}\right]$.

(II) l'application $\left.\mathcal{P}_{l_{1}}: \mathrm{x} \longrightarrow{ } \longrightarrow{ }_{l_{1}}\right)_{x}$, où $\left.\Theta_{l_{1}}\right)_{x}$ désigne l'application de $\underset{m}{E^{\prime}}$ dans $\underset{\mathrm{m}}{\mathrm{E}^{\prime \prime}}$ définie par $\left(Q_{\left.l_{1}\right)_{X}}\left(\sigma^{\prime}\right)=\mathrm{p}^{\prime \prime}\left(D_{X} \sigma^{\prime}\right)\right.$, est une l-forme sur $U$ à valeurs dans le fibré vectoriel $\left(E^{\prime}\right)^{*} \otimes E^{\prime \prime} \quad$ resp. 1'application $\varphi_{l_{2}}: x \longrightarrow\left(\Phi_{l_{2}}\right)_{X}^{\prime}$, où $\left(\mathscr{P} \ell_{2}\right)_{x}$ désigne l'application de $\underset{\mathrm{m}}{\mathrm{E}^{\prime \prime}}$ dans $\underset{\mathrm{m}}{\mathrm{E}}$ définie par $\left(P_{\ell_{2}}\right)_{X}\left(\sigma^{\prime \prime}\right)=p^{\prime}\left(D_{X} \sigma^{\prime \prime}\right)$, est une l-forme sur $U$ à valeurs dans le fibré vectoriel $\left.\left(E^{\prime \prime}\right)^{*} \otimes E^{\prime}\right]$. 
Soient, en effet, $X \in \underset{\text { min }}{T(U)}, \quad \sigma^{\prime} \in \underset{m}{E^{\prime}}$ et $f \in D(U)$ :
Puisque $D$ est une loi de dérivation sur $E$, on a

$$
\begin{aligned}
& D_{X}\left(f \sigma^{\prime}\right)=(X . f) \sigma^{\prime}+f D_{X} \sigma^{\prime}, \text { soit : } \\
& p^{\prime} D_{X}\left(f \sigma^{\prime}\right)=(X f) \cdot \sigma^{\prime}+f p^{\prime}\left(D_{X} \sigma^{\prime}\right) \\
& p^{\prime \prime} D_{X}\left(f \sigma^{\prime}\right)= f p^{\prime \prime}\left(D_{X} \sigma^{\prime}\right)
\end{aligned}
$$

$D^{\prime a u t r e ~ p a r t, ~ l ' a p p l i c a t i o n ~}\left(X, \sigma^{\prime}\right) \longrightarrow D_{X} \sigma^{\prime}$ est bi-additive en $X$ et en $\sigma^{\prime}$. On en déduit :

- d'une part que $D^{\prime}$ est une loi de dérivation sur E'

- d'autre part que $I^{\prime a p p l i c a t i o n ~}\left(X, \sigma^{\prime}\right) \longrightarrow p^{\prime \prime}\left(D_{X} \sigma^{\prime}\right)$ est $D(U)$-bilinéaire en $X$ et $\sigma^{\prime}$, donc que $P l^{l}$ est une l-forme sur $U$ ̀̀ valeurs dans $\left(E^{\prime}\right)^{*} \otimes E^{\prime \prime}$.

On a un résultat analogue en échangeant les rôles des ' et ", d'où la proposition.

Les lois de dérivation $D^{\prime}$ et $D^{\prime \prime}$, ainsi définies sur $E^{\prime}$ et $E^{\prime \prime}$, seront appelées "les projections" de $D$ sur $E^{\prime}$ et $E^{\prime \prime}$. [On dira aussi que $D^{\prime}$ est la projection de $D$ sur $E^{\prime}$ parallèlement à $\left.E^{\prime \prime}\right]$.

Les formes $Q \ell_{1}$ et $\Phi l_{2}$ seront appelées "les secondes formes fondamentales" de $E^{\prime}$ et $E^{\prime \prime}$ relativement à $D$. Cette terminologie sera justifiée au paragraphe $D$ de ce chapitre.

Remarque : la connexion $\tilde{D}$ sur $E$, obtenue à partir de $D^{\prime}$ et $D^{\prime \prime}$ en posant $\tilde{D}_{X^{\sigma}}=D^{\prime}{ }_{X}\left(p^{\prime} \sigma\right)+D_{X}^{\prime \prime}\left(p^{\prime \prime} \sigma\right)$, admet aussi $D^{\prime}$ et $D^{\prime \prime}$ comme projections; mais à des secondes formes fondamentales $\widetilde{\mathscr{Q}}_{l_{1}}$ et $\widehat{\mathscr{P}}_{l_{2}}$ nulles. Il est bien clair qu'une condition nécessaire et suffisante pour que $D=\tilde{D}$ est que $\rho_{l_{1}}=0$ et $\Theta_{l_{2}}=0$. 


\section{Formule des courbures}

Proposition III. A. 2

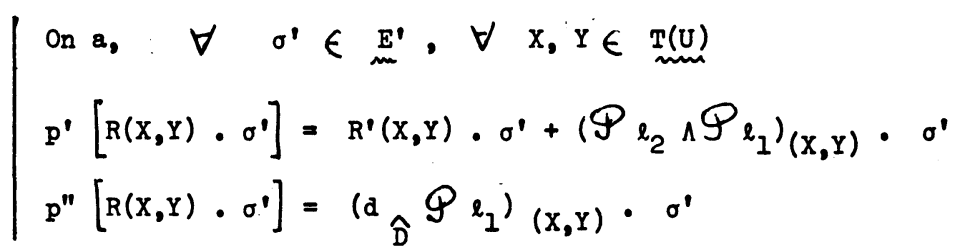

Dans ces équations,

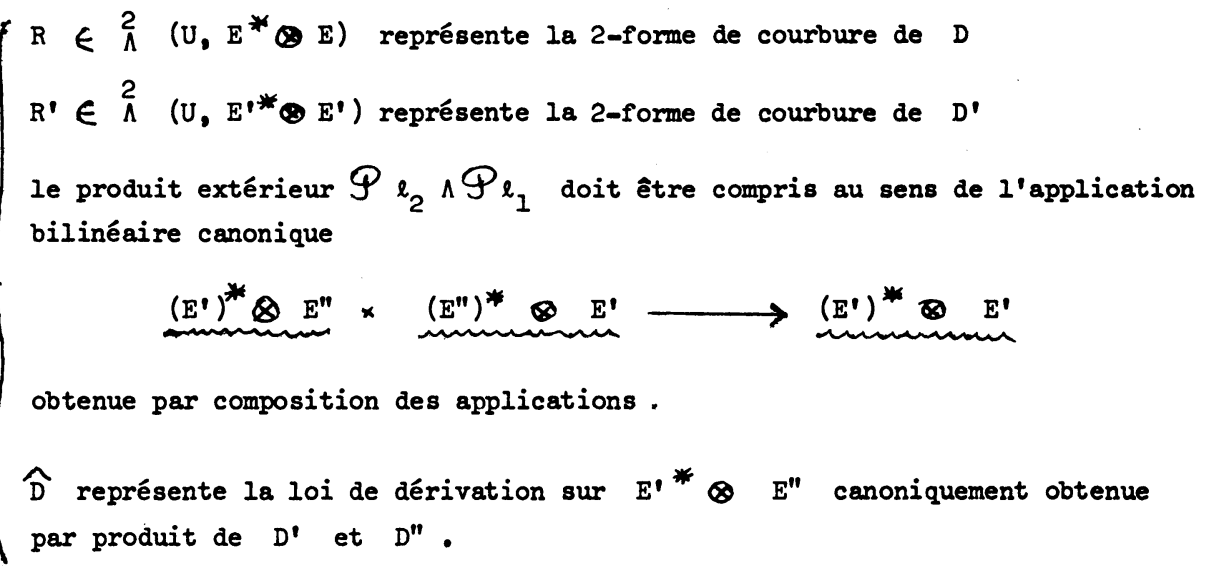

En abrégé, ces formules peuvent s'écrire :

$$
\begin{aligned}
& p^{\prime} R p^{\prime}=R^{\prime}+Q l_{2} \wedge Q_{\ell_{1}} \\
& p^{\prime \prime} R p^{\prime}=d_{\hat{D}} Q \ell_{1}
\end{aligned}
$$




$$
\begin{aligned}
\text { et, de même, } & \\
p^{\prime \prime} R p^{\prime \prime} & =R^{\prime \prime}+P_{\ell_{1}} \wedge Q_{l_{2}} \\
p^{\prime} R p^{\prime \prime} & =d_{\hat{D}} Q_{\ell}^{\ell}
\end{aligned}
$$

Démonstration : soient $X, Y \in T \underset{m}{T(U)}$ et $\sigma^{\prime} \in E^{\prime}$. On a alors :

$$
\begin{aligned}
& p^{\prime} R(X, Y) \cdot \sigma^{\prime}=p^{\prime}\left(D_{X} D_{Y} \sigma^{\prime}-D_{Y} D_{X} \sigma^{\prime}-D_{[X, Y]} \sigma^{\prime}\right) \\
& =p^{\prime} D_{X} p^{\prime} D_{Y} \sigma^{\prime}+p^{\prime} D_{X} p^{\prime \prime} D_{Y} \sigma^{\prime} \\
& \text { - } p^{\prime} D_{Y} p^{\prime} D_{X} \sigma^{\prime}-p^{\prime} D_{Y} p^{\prime \prime}, D_{X} \sigma^{\prime} \\
& \text { - } p^{\prime} D[X, Y] \quad \sigma^{\prime} \\
& =R^{\prime}(x, Y) \cdot \sigma^{\prime}+\left(\varphi_{l_{2}}\right)_{X}\left(\varphi_{l_{1}}\right)_{Y} \sigma^{\prime}-\left(\varphi_{l_{2}}\right)_{Y}\left(\varphi_{l_{1}}\right)_{X} \sigma^{\prime} \\
& \text { d'où la formule (I) } \\
& \text { et } \\
& p^{\prime \prime} R(X, Y) \cdot \sigma^{\prime}=p^{\prime \prime} D_{X} p^{\prime} D_{Y} \sigma^{\prime}+p^{\prime \prime} D_{X} p^{\prime \prime} D_{Y} \sigma^{\prime} \\
& \text { - } p^{\prime \prime} D_{Y} p^{\prime} D_{X} \sigma^{\prime}-p^{\prime \prime} D_{Y} p^{\prime \prime} D_{X} \sigma^{\prime} \\
& \text { - } p^{\prime \prime} D[X, Y] \sigma^{\prime} \\
& =\left(Q_{l_{1}}\right)_{X}\left(D^{\prime}{ }_{Y} \sigma^{\prime}\right)+D_{X}^{\prime \prime}\left[\left(\rho_{l_{1}}\right)_{Y} \sigma^{\prime}\right] \\
& -\left(\mathcal{\varphi}_{l_{1}}\right)_{Y}\left(D^{\prime}{ }_{X} \sigma^{\prime}\right)-D_{Y}^{\prime \prime}\left(\mathscr{P}_{l_{1}}\right)_{X} \sigma^{\prime} \\
& \left.-\left(\varphi_{l_{1}}\right)_{[\mathrm{X}, \mathrm{y}}\right]^{\sigma^{\prime}} \\
& \left.\left.=D_{X}\left(\varphi_{l_{1}}\right)_{Y} \cdot \sigma^{\prime}-D_{Y}\left(Q_{l_{1}}\right)_{X} \cdot \sigma^{\prime}-Q_{l_{1}}\right)_{[X, Y}\right]^{\cdot \sigma^{\prime}} \\
& =\left(d \hat{D} \Phi_{\ell_{l}}\right)(X, Y) \cdot \sigma^{\prime} \\
& \text { d'où la formule (II). }
\end{aligned}
$$


Cas où $\mathrm{D}$ respecte une pseudométrique sur $\mathrm{E}$

Supposons avoir défini, pour tout point $x$ de $U$, une forme bilinéaire symétrique non dégénérée $g_{x}$ sur $E_{x}$, de telle façon que l'application $x \longrightarrow B_{x}(\sigma(x), \tau(x))$ soit différentiable quelles que soient $\sigma, \tau \in \underset{\mathrm{m}}{\mathrm{E}}$.

$$
\text { Supposons que la section } x \longrightarrow B_{x} \text { de } E^{*} \otimes E^{*} \text { ainsi }
$$
définie ait une dérivée covariante nulle, relativement à une connexion $D$ sur E :

$$
\begin{aligned}
\left(D_{X} g\right)(\sigma, \tau) & =X \cdot g(\sigma, \tau)-g\left(D_{X} \sigma, \tau\right)-g\left(\sigma, D_{X} \tau\right) \\
& =0 \quad \forall X \in \underset{m}{T(U)} \forall \sigma, \tau \in \underset{m}{E} .
\end{aligned}
$$

Supposons enfin que $E^{\prime} \longrightarrow U$ soit un sous-fibré vectoriel de $E$ tel que, pour tout $x \in U, E^{\prime} x$ soit un sous-espace non isotrope de $E_{x}$ relativement à $B_{x}$ (ce qui signifie que la forme bilinéaire symétrique $g^{\prime}{ }_{x}$, induite par $g_{x}$ sur $E_{x}^{\prime}$, est non dégénérée). Soit $g^{\prime}$ la métrique ainsi définie sur $E^{\prime}$.

$$
\text { L'orthogonal } E^{\prime \prime} \text { de } E^{\prime}{ }_{x} \text { dans } E_{x} \text { est alors un supplémentaire }
$$

de $E^{\prime} x$ dans $E^{\prime \prime}{ }_{x}$, et la forme bilinéaire symétrique $g^{\prime \prime}$ induite par $g_{x} \operatorname{sur} E^{\prime \prime}$ est également non dégénérée. Soit $g^{\prime \prime}$ la métrique ainsi définie sur $E^{\prime \prime}=\bigsqcup_{x \in U} E^{\prime \prime}{ }_{x}$.

\section{Proposition III. A. 3}

$D^{\prime}$ et $D^{\prime \prime}$ désignant les projections de $D$ sur $E^{\prime}$ et $E^{\prime \prime}$, on a :

$$
D^{\prime}{ }_{X} g^{\prime}=0 \quad \text { et } \quad D_{X} g^{\prime \prime}=0 \quad \forall \quad X \in T(U) \cdot .
$$


En effet $\left(D^{\prime} \mathrm{X}^{\prime}\right)\left(\sigma^{\prime}, \tau^{\prime}\right)=X \cdot B^{\prime}\left(\sigma^{\prime}, \tau^{\prime}\right)-g^{\prime}\left(D^{\prime} X \sigma^{\prime}, T^{\prime}\right)-g^{\prime}\left(\sigma^{\prime}, D^{\prime} X T^{\prime}\right)$

Mais $g^{\prime}\left(\sigma^{\prime}, \tau^{\prime}\right)=g\left(\sigma^{\prime}, \tau^{\prime}\right)$

et

$$
\begin{aligned}
g^{\prime}\left(D_{X}^{\prime} \sigma^{\prime}, \tau^{\prime}\right) & =g\left(p^{\prime} D_{X} \sigma^{\prime}, \tau^{\prime}\right) \\
& =g\left(D_{X} \sigma^{\prime}, \tau^{\prime}\right)
\end{aligned}
$$

puisque $E^{\prime}$ et $E^{\prime \prime}$ sont orthogonaux relativement à $\mathbf{g}$.

Donc $\left(D^{\prime} B^{\prime}\right)\left(\sigma^{\prime}, \tau^{\prime}\right)=\left(D_{X} g\right)\left(\sigma^{\prime}, \tau^{\prime}\right)=0$

De même, $D^{\prime \prime}{ }_{X}^{\prime \prime}=0$ d'où la proposition.

Sous les hypothèses précédentes $\left(D_{B}=0, E^{\prime}\right.$ et $E^{\prime \prime}$ orthogonaux relativement $\grave{a}$ ), la donnée de chacune des deux formes $\Phi_{l}$ et $\Phi_{\ell}$ est équivalente à la donnée de l'autre. Plus précisément, on a la

\section{Proposition III. A. 4}

$\forall x \in \underset{m}{T(U)}$. I'application $\left(\mathcal{P}_{l_{2}}\right)_{X}$ de $\underset{\mathrm{m}}{E^{\prime \prime}}$ dans $\underset{\mathrm{m}}{E^{\prime}}$ est égale à I'opposée de la transposée de $\left(\rho_{\ell}{ }_{1}\right)_{X}$, relativement aux métriques $g^{\prime}$ et $g^{\prime \prime}$ c'est-à-dire : $g\left(\left(\varphi_{l_{1}}\right)_{X} \sigma^{\prime}, \delta^{\prime \prime}\right)=-g\left(\left(\varphi_{l_{2}}\right)_{X} \sigma^{\prime \prime}, \sigma^{\prime}\right) \quad \forall \sigma^{\prime} \in \underline{m}^{\prime}, \forall \sigma^{\prime \prime} \in \underset{m}{E^{\prime \prime}}$.

En effet $g\left(\left(Q_{l_{1}}\right)_{X} \sigma^{\prime}, \sigma^{\prime \prime}\right)=g\left(p^{\prime \prime} D_{X} \sigma^{\prime}, \sigma^{\prime \prime}\right)$

$$
=B\left(D_{X} \sigma^{\prime}, \sigma^{\prime \prime}\right) \begin{aligned}
& \text { puisque } E^{\prime} \text { et } E^{\prime \prime} \text { sont } \\
& \text { orthogonaux, }
\end{aligned}
$$

$=X \cdot B\left(\sigma^{\prime}, \sigma^{\prime \prime}\right)-g\left(D_{X} \sigma^{\prime \prime}, \sigma^{\prime}\right)$ puisque $D_{X} B=0$, 


$$
\begin{gathered}
=-B\left(D_{X} \sigma^{\prime \prime}, \sigma^{\prime}\right) \begin{array}{l}
\text { puisque } \sigma^{\prime} \text { et } \sigma^{\prime \prime} \\
\text { sont orthogonales }
\end{array} \\
=-B\left(p^{\prime} D_{X} \sigma^{\prime \prime}, \sigma^{\prime}\right) \begin{array}{l}
\text { puisque } E^{\prime} \text { et } E^{\prime \prime} \\
\text { sont orthogonaux.- }
\end{array} \\
\text { C. Q. F. D. }
\end{gathered}
$$

\section{B - Transcription en termes de fibrés principaux}

$$
\text { Soient } k \text { et } n \text { deux entiers }>0(k<n) \text {. Notons }\left\{e_{1} \ldots, e_{n}\right\}
$$

la base canonique de $\mathbb{R}^{n}$; identifions $\left\{e_{1}, \ldots, e_{k}\right\}$ à la base canonique de $R^{k}$, et $\left\{e_{k+1}, \ldots, e_{n}\right\}$ à la base canonique de $\mathbb{R}^{n-k}$, de telle sorte que $\mathbb{R}^{n}$ est identifié à $\mathbb{R}^{k} \oplus \mathbb{R}^{n-k}$ et que $G L(k, R) \times G L(n-k, R)$ est identifié à un sous-groupe de Lie de $G L(n, R)$.

On supposera désormais que $G$ est un sous-groupe de Lie de $G L(n, \mathbb{R})$ et que $H=G \cap[G L(k, \mathbb{R}) \times G L(n-k, \mathbb{R})]$ Identifiant de façon neturelle $G L(k, R)$ et $G L(n-k, R)$ à des sous-groupes de $G L(k, R) \times G L(n-k, \mathbb{R})$, supposons ${ }^{(1)}$ :

$$
H=H^{\prime} \times H^{\prime \prime} \quad \text { avec } H^{\prime}=G \cap G L(k, R) \text { et } H^{\prime \prime}=G \cap G L(n-k, \mathbb{R}) \text {. }
$$

On a alors $\underline{H}^{\prime}=\underline{G} \bigcap^{\text {End }} \mathbf{R}^{k}$ et $\underline{H}^{\prime \prime}=\underline{G} \bigcap^{\text {End } \mathbb{R}^{n-k}}$

Posons $\left.d b_{1}=\underline{G} \cap\left(\left(\mathbb{R}^{k}\right)_{\mathbb{R}}^{*} \mathbb{R}^{n-k}\right)\right)$ et $d t_{2}=G \cap\left(\left(\mathbb{R}^{n-k}\right)^{*} \underset{\mathbb{R}}{\otimes} \mathbb{R}^{k}\right)$,
(1) Il en est ainsi par exemple pour
$G=O(n, \mathbb{R}), G L(n, \mathbb{R})$, etc... 
[End $\mathbf{R}^{\mathrm{n}}$ étant naturellement identifié à la somme directe End $\mathbb{R}^{k} \oplus$ End $\left.\mathbb{R}^{n-k} \oplus\left(\left(\mathbb{R}^{k}\right)^{*} \underset{\mathbb{R}}{\otimes} R^{n-k}\right) \oplus\left(\left(\mathbb{R}^{n-k}\right) \underset{\mathbb{R}}{\otimes} \mathbb{R}^{k}\right)\right]$. On vérifie aisément :

$$
\begin{aligned}
& \operatorname{ad}\left(\mathrm{H}^{\prime}\right) \cdot \underline{\mathrm{H}^{\prime \prime}}=0 \\
& \operatorname{ad}\left(H^{\prime}\right) \cdot d b_{1} c d b_{1} \\
& \operatorname{ad}\left(H^{\prime}\right) \cdot d b_{2} \subset d b_{2} \\
& {\left[\mathrm{db} b_{1}, \mathrm{db}_{2}\right]<\mathrm{H}} \\
& \operatorname{ad}\left(H^{\prime \prime}\right) \cdot \underline{H}^{\prime}=0 \\
& \operatorname{ad}\left(H^{\prime \prime}\right) \cdot d b_{1} c d b_{1} \\
& \operatorname{ad}\left(\mathrm{H}^{\prime \prime}\right) \cdot \mathrm{db} \mathrm{b}_{2} \mathrm{CCl} b_{2} \\
& {\left[d b_{1}, d b_{1}\right]=0 ;\left[d b_{2}, d b_{2}\right]=0}
\end{aligned}
$$

Si a $E \underline{G}$, on notera $a_{\underline{H}}, a_{\underline{H}}$, a $a_{b_{1}}$ et a ${ } b_{2}$ ses composantes relativement à la décomposition $\underline{G}=\underline{H}^{\prime} \oplus \underline{\mathrm{H}}^{\prime \prime} \oplus \mathrm{db}_{1} \oplus \mathrm{db}{ }_{2}$, et on posera $d b=d b_{1}+d b_{2}$

$$
{ }^{a} d b=a_{d b}+a d b_{2}
$$

(on a clairement ad(H) db c clb et $[\mathrm{dlb}, d b] \subset \underline{\mathrm{H}}$ ).

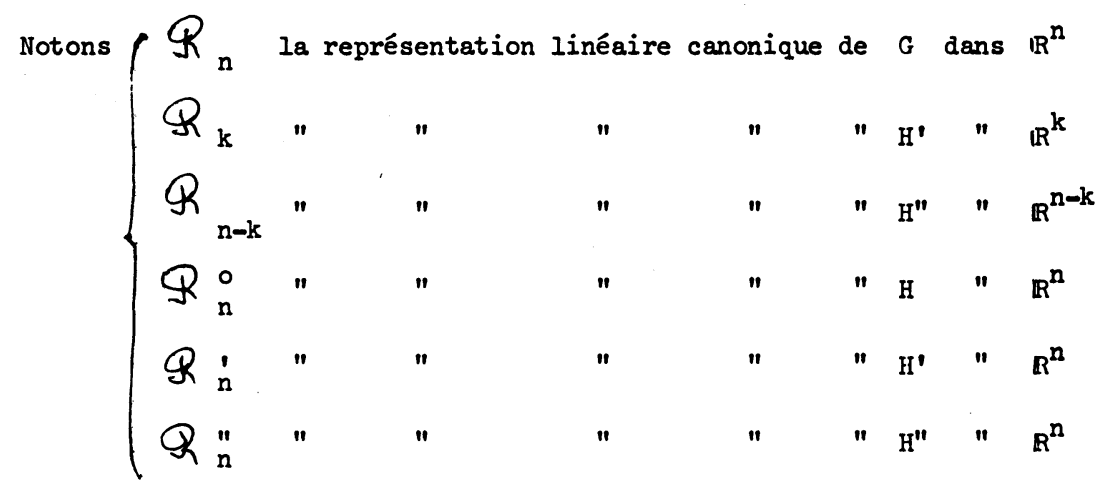




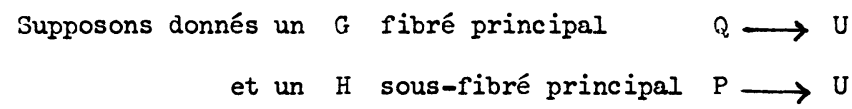

Puisque $H^{\prime}=H^{\prime} \times H^{\prime \prime}, P$ est égal au produit fibré $P^{\prime} P^{\prime \prime}$ du $H^{\prime}$-fibré principal $\mathrm{P}^{\prime}=\mathrm{P} / \mathrm{H}^{\prime \prime} \longrightarrow \mathrm{U}$ et du $\mathrm{H}^{\prime \prime}$ - fibré principal

$\mathrm{P}^{\prime \prime}=\mathrm{P} / \mathrm{H}^{\prime} \rightarrow \mathrm{U} \quad$ (II. B. I).

Lemme III. B. I

$\mathbb{R}_{\mathbb{R}_{\mathrm{n}}}^{\mathrm{n}}[\mathrm{Q}]=\mathbb{R}_{\mathbb{R}_{\mathrm{k}}^{\mathrm{k}}}\left[\mathrm{P}^{\prime}\right] \oplus \mathbb{R}_{\mathbb{R}_{\mathrm{n}-\mathrm{k}}^{\mathrm{n}-\mathrm{k}}}^{\left[\mathrm{P}^{\prime \prime}\right]}$

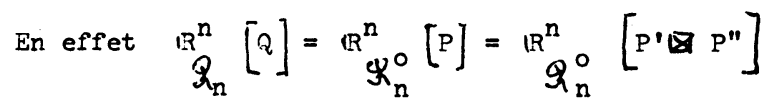

Mais puisque $\mathbb{R}^{n}=\mathbb{R}^{k} \oplus \mathbb{R}^{n-k}$ et puisque

$$
\begin{aligned}
Q_{n}^{\circ} & =Q_{k} \oplus Q_{n-k}, \\
\mathbb{R}_{M_{n}^{0}}^{n}\left[P^{\prime} \otimes P^{\prime \prime}\right] & =\mathbb{R}_{R_{k}^{k}}^{k}\left[P^{\prime}\right] \oplus \mathbb{R}_{R^{\prime}-k}^{n-k}\left[P^{\prime \prime}\right],
\end{aligned}
$$

d'où le lemne

$\mid \begin{aligned} & \text { Notons désormais } E, E^{\prime} \text { et } E^{\prime \prime} \text { les fibrés vectoriels } \mathbb{R}_{\mathbb{R}_{n}}^{n}[Q] \text {. } \\ & \mathbb{R}_{\mathbb{R}_{k}^{k}}\left[\mathrm{P}^{\prime}\right] \text { et } \quad \mathbb{R}_{\mathbb{R}_{n-k}^{n-k}}\left[\mathrm{P}^{\prime \prime}\right]\end{aligned}$

Remarque

Supposons réciproquement que $E=\mathbb{R}_{\mathbb{R}_{n}}^{n}[Q]$ admet une décomposition

$E^{\prime} \oplus E^{\prime \prime}$ en somme de WHITHEY. 


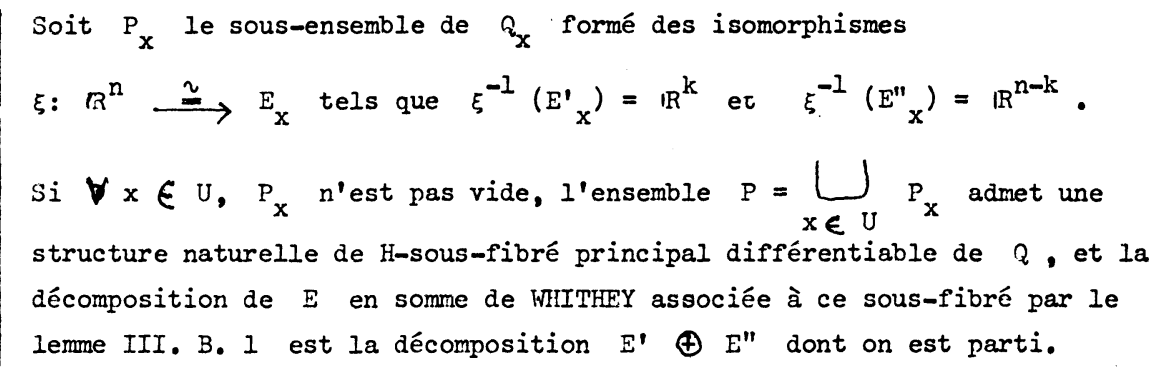

Il en est en particulier ainsi dans le cas où $Q$ est l'espace des repères orthonormés pour une métrique pseudo-riemannienne $g$ sur $E$, et où $E^{\prime}$ $E^{\prime \prime}$ sont orthogonaux relativement à $g$.

\section{Lemme III. B. 2}

Nous avons vu que les sous-espaces $d b_{1}$ et $d b_{2}$ de $G$ sont stables par la représentation adjointe ad de $\mathrm{H}$ dans $\mathrm{G}$

Le fibré vectoriel $\left(c b_{1}\right)_{\text {ad }}[P]\left(\operatorname{resp} .\left(d b_{2}\right)_{\text {ad }}[P]\right)$ est un sous-fibré vectoriel de $\left.\left(E^{\prime}\right)^{*} \otimes E^{\prime \prime} \quad\left(\operatorname{resp} .\left(E^{\prime \prime}\right)^{*} \otimes E^{\prime}\right)\right)$.

En effet, l'injection canonique $i$ de $d l_{I}=\underline{G} \cap\left(\left(R^{k}\right) \underset{R}{\otimes} \mathbb{R}^{n-k}\right)$ dans $\left(\mathbb{R}^{k}\right) \bigotimes_{R} \mathbb{R}^{n-k}$ induit, dans la catégorie $\zeta_{I I}$, un morphisme injectif de $\left(d b_{1}, a d\right)$ dans $\left(\left(\mathbb{R}^{k}\right) \underset{R}{\otimes} \mathbb{R}^{n-k}, \mathbb{R}_{k}^{*} \otimes \mathbb{R}_{n-k}\right)$.

Par application du foncteur modelage $\mu_{p}$, qui est exact, on en déduit une injection $\bar{i}$ de $\left(A_{1}\right)_{\text {ad }}[P]$ dans $\left(E^{\prime}\right)^{*} \otimes E^{\prime \prime}$. On définit de même $\bar{j}:\left(d b_{2}\right)_{a d}[P] \longrightarrow\left(E^{\prime \prime}\right)^{*} \otimes E^{\prime}$. 
Soient désormais $Q, P, P^{\prime}, P^{\prime \prime}, E, E^{\prime}, E^{\prime \prime}$ définis comme précédemment, et soit $\omega$ une connexion sur $Q$. Puisque ad(H)db $C \mathrm{clb}$, $\omega$ induit une connexion $\tilde{\omega}=(\omega \mid P)_{\underline{H}}$ sur $P$ (théorème II. A. 1), qui est le produit d'une connexion $\omega^{\prime}$ sur $P^{\prime}$ et d'une connexion $\omega^{\prime \prime}$ sur P" (Proposition II. B. 1). La forme de plongement $\mathrm{Pl}=(\omega \mid \mathrm{P})_{d l}$ admet, par projections de db sur $d b_{1}$ et $d b_{2}$, deux composantes

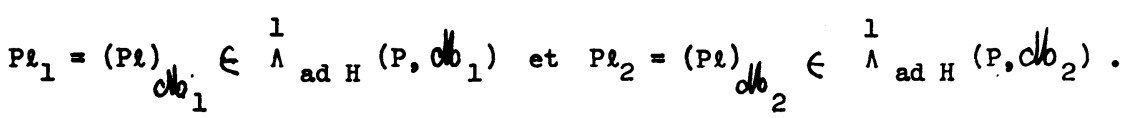
Notons D la connexion sur E associée à la connexion $\omega$.

Théorème III. B. 3

(I) Les connexions $D^{\prime}$ sur $E^{\prime}$ et $D^{\prime \prime}$ sur $E^{\prime \prime}$, respectivement associées à $\omega^{\prime}$ et $\omega^{\prime \prime}$, sont les projections de $D$ sur $E^{\prime}$ et $E^{\prime \prime}$.

(II) Les 1-formes $Q_{\ell_{1}} \in \stackrel{1}{\Lambda}\left(\cdot U,\left(c b_{1}\right)_{\text {ad }}[P]\right)$ et $Q_{l_{2}} \in \Lambda\left(U,\left(d^{d} b_{2}\right)_{a d}[P]\right)$, respectivement associées à $P l_{1}$ et $\mathrm{Pl}_{2}$ par $\lambda_{1}$ (théorème I. B. 2), sont les secondes formes fonda.. mentales de $E^{\prime}$ et $E^{\prime \prime}$ relativement à $D$ une fois $\left(d b_{1}\right)_{\text {ad }}[P]$ et $\left(d b_{2}\right)_{\text {ad }}[P]$ identifiés à des saus-fibrés vectoriels de $\left(E^{\prime}\right)^{*} \otimes E^{\prime \prime}$ et $\left.\left(E^{\prime \prime}\right)^{*} \otimes E^{\prime}\right)$.

\section{Démonstration :}

Notons $\eta$ l'injection de $P$ dans $Q$, et soit $\xi=\eta\left(\xi_{0}\right)$ un élément de $\tau(P)$. 


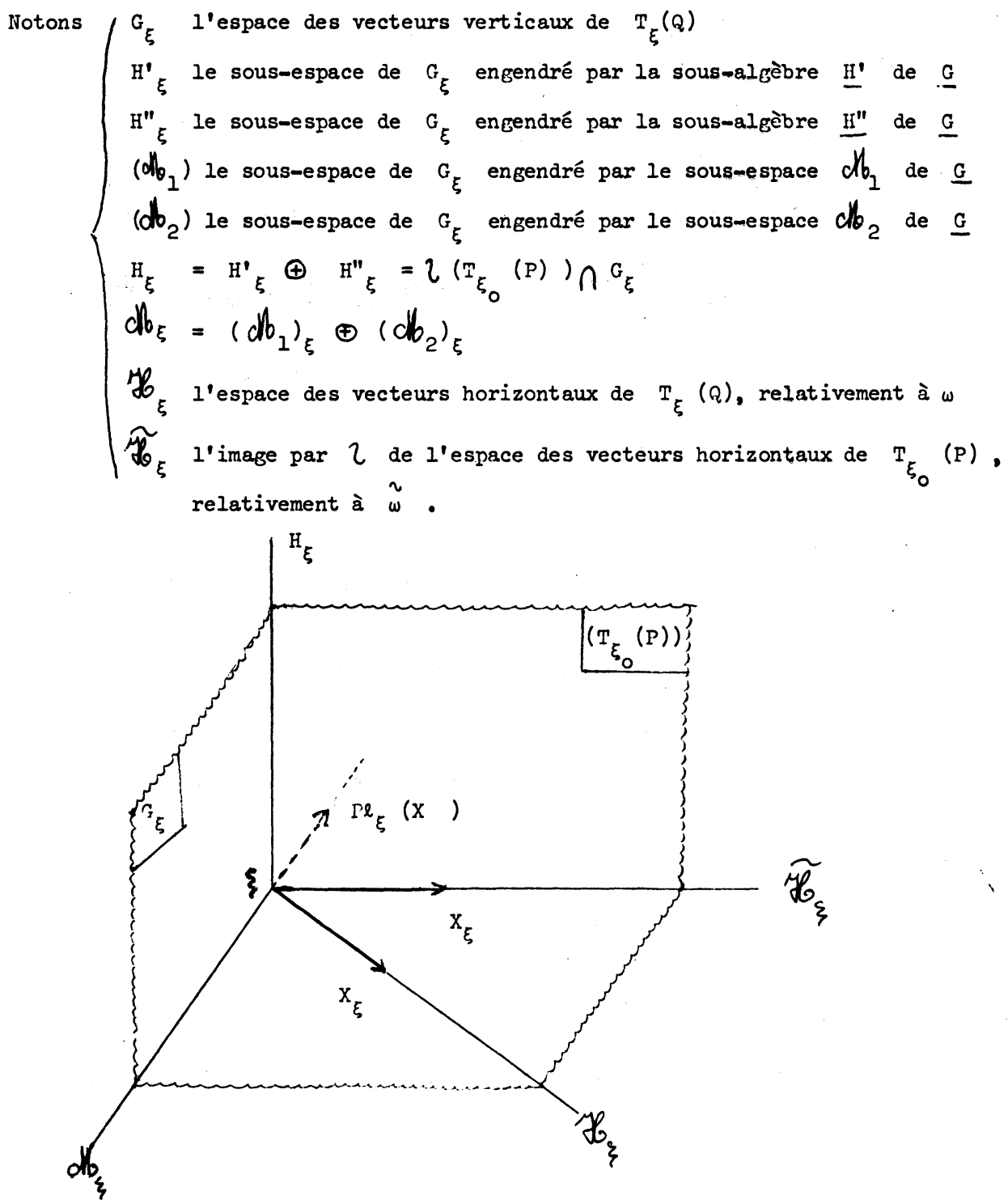

Schéma de $\mathrm{T}_{\xi}(0)$ 
On a évidemment $G_{\xi}=H_{\xi} \oplus d b_{\xi}$, et puisque $\omega \eta=\tilde{\omega}+\mathrm{Pl}$, $d b_{\xi}+b_{\xi}=d b_{\xi}+\widetilde{b}_{\xi}$. Pour tout $x \in \underset{m}{T(U)}$, notons $x *$ le relèvement horizontal de $X$ dans $Q$ (relativement à $\omega$ ), et $\bar{X}^{*}$ l'image par $Z$ du relèvement horizontal de $X$ dans $P$ (relativement $\grave{a} \underset{\omega}{\eta}$ ). Si $\sigma \in \underset{w}{E}$, notons $\tilde{\sigma}$ l'élément correspondant de

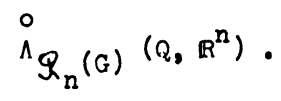

Les fonctions $\Psi \in{\stackrel{\circ}{\Lambda} \mathscr{Q}_{n}(G)}\left(Q, \mathbb{R}^{n}\right)$ sont entièrement déterminées par leur restriction à $\chi(P)$. Celles qui correspondent aux sections $\sigma^{\prime}$ (resp. $\sigma^{\prime \prime}$ ) du sous-fibré $E^{\prime}$ (resp. $E^{\prime \prime)}$ de $E$, sont celles dont la restriction à $\chi(P)$ prend ses valeurs dans le sous-espace $\mathbb{R}^{k}$ (resp. $\left.\mathbb{R}^{n-k}\right)$ de $R^{n}$.

D'après le théorème I. C. I, $\widetilde{D_{X} \sigma^{\prime}}=X^{*} . \tilde{\sigma}^{\prime} \quad \forall x \in \underset{T(U)}{T(U)}$ $\forall \sigma^{\prime} \in \underset{m}{E^{\prime}} \subset \underset{m}{E}$. Remarquons la valeur en $\xi$ du champ de vecteurs $X^{*}-\bar{X}^{*}$ est égale à l'opposé du vecteur $P \ell_{\xi}\left(\bar{X}^{*}\right)$ engendré en $\xi$ par l'élément $\mathrm{Pl}\left[\mathcal{l}^{-1}\left(\overline{\mathrm{X}}_{\xi}^{*}\right)\right]$ de $\mathrm{dlb}$.

$$
\text { Remarquons enfin que, pour } \varphi \in{\stackrel{\circ}{\Lambda} Q_{n}(G)}\left(Q, R^{n}\right) \text {, la dérivée }
$$

$A_{\xi} \cdot \Psi$ de $\varphi$, relativement au vecteur vertical $A_{\xi} \in T_{\xi}(Q)$ engendré par l'élément $A$ de $G$, est égal à $-\langle A, \varphi(\xi)\rangle$ où $\langle$,$\rangle désigne l'ap-$ plication bilinéaire canonique $G \times R^{n} \longrightarrow R^{n}:$ en effet le groupe des translations à droite (expt $A)_{t \in A}$ est le groupe à un paramètre de transformations de $Q$ engendrant le champ fondamental $\xi \longrightarrow A_{\xi}$; on a donc $\quad A_{\xi} \cdot \varphi=\lim _{t \rightarrow 0} \frac{1}{t}[\varphi(\xi \cdot \exp t A)-\varphi(\xi)]$. 


$$
\begin{aligned}
& \text { Mais } \Psi(\xi \cdot \exp t A)=Q_{n}\left((\exp t A)^{-1}\right) \cdot(\varphi(\xi))=Q_{n}(\exp -t A) \cdot(\varphi(\xi)) \\
& \text { et } A_{\xi} \cdot \varphi=Q_{n}\left(\lim _{t \rightarrow 0} \frac{1}{t} \exp (-t A)-(i d)\right) \cdot(\varphi(\xi)) \\
& =-\langle A, \varphi(\xi)\rangle \text {. }
\end{aligned}
$$

On a donc $\widetilde{\left(D_{X} \sigma^{\prime}\right)}(\xi)=X_{\xi}^{*} \cdot \widetilde{\sigma^{\prime}}=\bar{X}_{\xi}^{*} \cdot \widetilde{\sigma^{\prime}}-P_{\xi}\left(\bar{X}^{*}\right) \cdot \widetilde{\sigma^{\prime}}$

$$
=\bar{x}_{\xi^{*}}^{*} \tilde{\sigma}^{\prime}+<\operatorname{Pl}\left(\imath^{-1}\left(\bar{X}_{\xi}^{*}\right)\right), \tilde{\sigma}^{\prime}(\xi)>\text {. }
$$

Observant

- d'une part que $\bar{X}_{\xi}^{*} \cdot \tilde{\sigma}^{\prime}=\left(\widetilde{D^{\prime}{ }_{X}^{\prime}} \sigma^{\prime}\right)(\xi)$ est en particulier un élément de $\mathbb{R}^{k}$,

- d'autre part que $\left\langle\operatorname{Pl}\left(\eta^{-1}\left(\bar{x}_{\xi}^{*}\right)\right), \tilde{\sigma}^{\prime}(\xi)\right\rangle=\left\langle P l_{1}\left(2^{-1}\left(\bar{X}_{\xi}^{*}\right)\right), \tilde{\sigma}^{\prime}(\xi)\right\rangle$

$$
\text { est un élément de } \mathbb{R}^{\mathrm{n}-\mathrm{k}} \text {, car }<\mathrm{d}_{1}, \mathbb{R}^{\mathrm{k}}>\mathbb{C} \mathbb{R}^{\mathrm{n}-\mathrm{k}} \text { et }
$$

$<x_{2}, \mathbb{R}^{k}>=0$,

on en déduit : $D^{\prime}{ }_{X} \sigma^{\prime}=p^{\prime}\left(D_{X} \sigma^{\prime}\right)$

$$
\left(\varphi_{l_{1}}\right)_{X}\left(\sigma^{\prime}\right)=p^{\prime \prime}\left(D_{X} \sigma^{\prime}\right)
$$

et, de même : $D^{\prime \prime} \sigma^{\prime \prime}=p^{\prime \prime}\left(D_{X} \sigma^{\prime \prime}\right)$

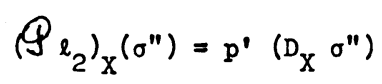

$$
\forall \sigma^{\prime \prime} \in \underset{m}{E^{\prime \prime}}
$$

d'où le théorème. 
C - Connexions canoniques sur les variétés de Stiefel généralisées

Soit $G$ un sous-groupe de Lie de $G L(n, \mathbb{R})$. Conservant les conventions du paragraphe $B$, on pose : $H^{\prime}=G \cap G L(k, \mathbb{R}) H^{\prime \prime}=G \cap G L(n-k, \mathbb{R})$ $H=G \cap(G L(k, R) \times G L(n-k, R))$ et $I^{\prime}$ on suppose toujours $H=H^{\prime} \times H^{\prime \prime}$. on identifie $G$, de façon naturelle, à une famille de repères de $R^{n}$ qui seront dits "adaptés" (sous-entendu : à la G-structure plate canonique de $\mathbb{R}^{\mathrm{n}}$ ).

On appellera grassmannienne $G_{k, n-k}$ l'espace homogène symétrique G H'H II" : géométriquement, un point de $G_{k, n-k}$ est.t un couple $\left(\xi, \xi^{\prime}\right)$ formé $d^{\prime}$ un $k-p l a n \quad \xi$ de $\mathbb{R}^{n}$ et d'un $(n-k)$ plan supplémentaire $\xi^{\prime}$ tels qu'il existe un repère adapté $g \in G$ de $\mathbb{R}^{\mathrm{n}}$ dont les $k$ premiers vecteurs sont dans $\xi$ et les $n-k$ derniers dans $\xi^{\prime}$.

On appellera variété de Stiefel $v_{k, n-k}$ (resp. $v_{n-k, k}$ ) le H'-fibré principal $G / H^{\prime \prime}$ considéré comme fibré au-dessus de la grassmannienne (resp. le $H^{\prime \prime}$ fibré principal $\left.G / H^{\prime} \longrightarrow G / H^{\prime} \times H^{\prime \prime}\right):$ géométriquement, la fibre de $v_{k, n-k}$ (resp. $v_{n-k, k}$ ) au-dessus $d^{\prime} u n$ couple $\left(\xi, \xi^{\prime}\right) \in G_{k, n-k}$ est composée de l'ensemble des repères de $\xi$ (resp. $\xi$ ') qui possèdent la propriété de pouvoir être complétés par un repère de $\xi^{\prime}$ (resp. $\xi$ ) pour former un repère adapté $g \in G$ de $R^{n}$.

On notera $E_{k}^{n}$ (resp. $E_{n-k}^{n}$ ) le fibré vectoriel modelé sur $v_{k, n-k}$ (resp. $v_{n-k, k}$ ), de base $G_{k, n-k}$ :

$$
\left\{\begin{array}{l}
E_{k}^{n}=\mathbb{R}_{Q_{k}^{k}}^{k}\left[v_{k, n-k}\right] \\
E_{n-k}^{n}=R_{Q_{n-k}^{n-k}}^{n}\left[v_{n-k, k}\right]
\end{array}\right.
$$


Géométriquement, la fibre de $F_{k}^{n}$ (resp. $E_{n-k}^{n}$ ) au-dessus du couple $\left(\xi, \xi^{\prime}\right)$ est $I^{\prime e s p a c e}$ vectoriel $\xi\left(\right.$ resp. $\left.\xi^{\prime}\right)$. Puisque $\xi \oplus \xi^{\prime}=\mathbb{R}^{\mathrm{n}}$, la somme de WHITNEY $E_{k}^{n} \oplus E_{n-k}^{n}$ est égale au fibré vectoriel trivial $R^{n} \times G_{k, n-k} \rightarrow G_{k, n-k} \cdot$

Par projection sur $\mathbb{E}_{k}^{n}$ parallèlement à $E_{n-k}^{n}$ de la connexion naturelle à courbure nulle du fibré vectoriel trivial $R^{n} \times G_{k, n-k} \rightarrow G_{k, n-k}$, on obtient une connexion $D^{k}$ qu'on appellera la connexion canonique de $E_{k}^{n}$.

Soit $Q$ le G-fibré principal trivial $G \times G_{k, n-k} \rightarrow G_{k, n-k}$ et $\omega$ sa connexion canonique à courbure nulle. Soit $P$ le $H^{\prime} \times H^{\prime \prime}$ fibré principal $G \longrightarrow G H^{\prime} \times H^{\prime \prime}$. Définissant $d b=d b_{1} \oplus d b_{2}$ comme au paragraphe $B$ de ce chapitre, la connexion $\omega_{0}$ induite sur $P$ par $(\omega, d b)$ est la connexion canonique de la grassmannienne munie de sa structure d'espace homogène symétrique (cf. II. C. exemple 4). D'après II. B. I, $P$ est le produit fibré $v_{k, n-k} v_{n-k, k}$ et $\omega_{0}$ est le produit d'une connexion $\omega_{k}$ sur $v_{k, n-k}$ et d'une connexion $\omega_{n-k}$ sur $v_{n-k, n}$. D'après le théorème III. B. 3 , la connexion canonique $D_{k}$ est la connexion sur $E_{k}^{n}$ associée à $\omega_{k}$.

\section{Remarques}

1) Dans le cas $G=O(n),\left(\xi, \xi^{\prime}\right) \in G_{k, n-k}$ implique que $\xi^{\prime}$ est le supplémentaire orthogonal de $\xi$ : on peut alors identifier $G_{k, n-k}$ à l'ensemble des $k$-plans (ou des $n-k$ plans) de $\mathbb{R}^{n}$. La définition de $\omega_{k}$ est alors donnée dans Sh. KOBAYASHI [9], NARASIMHAN et RAMANAN [19], et TAKIZAWA [23].

2) Une importante propriété de $D_{k}$ (cf. Chapitre IV) est d'être obtenue comme projection de la connexion canonique à courbure nulle d'un fibré trivial. 
Interprétation géométrique du fibré tangent à la grassmannienne :

Puisque la forme de plongement $Q_{\ell \in \Lambda^{1}}$ ad $\left(P, b_{1} \oplus d b_{2}\right)$ de $P$ dans $(Q, \omega)$ n'est autre que la forme fondamentale de $P$ considéré comme espace de repères au-dessus de la grassmannienne (II. C. exemple 4), l'application $\mathrm{Pl}: \mathrm{T}\left(G_{k, n-k}\right) \simeq\left(d b_{1}[P]+d b_{2}[P]\right)$ est un isomorphisme de fibrés vectoriels, et par conséquent un vecteur $\left.x \in T(\xi, \xi)^{\prime}\right)\left(G_{k, n-k}\right)$ s'interprète comme un élément de $\quad\}^{*} \otimes \xi^{\prime} \oplus,(\xi \cdot)^{*} \otimes$

Dans le cas particulier $G=O(n)$, il suffit de connaître $Q_{l_{1}}$ pour connâ̂tre $Q_{l}=Q_{l_{1}}+Q_{l_{2}}$ (d'après III. A. 4), observant enfin que $d b_{1}$ est alors égal à $\left(\mathbb{R}^{k}\right)_{\mathbb{R}}^{*}\left(\mathbb{R}^{n-k}\right)$ tout entier, on obtient le

Théorème III. C. I

Notant $\mathbb{G}_{k, n-k}, \mathbb{W}_{k, n-k}, \mathbb{E}_{k}^{n}$ les espaces $G_{k, n-k}, v_{k, n-k}$ et $E_{k}^{n}$ dans le cas $G=O(n)$, et notant $Q_{l_{1}}$ et $\Phi_{l_{2}}$ les secondes formes fondamentales de $\mathbb{E}_{k}^{n}$ et $\mathbb{E}_{n-k}^{n}$ relativement à la connexion canonique à courbure nulle du fibré trivial $\mathbb{R}^{n} \times \mathbb{G}_{k, n-k} \longrightarrow \mathbb{G}_{k, n-k}$

$$
\text { les applications } \begin{aligned}
& \mathcal{P}_{\ell_{1}}: T\left(\mathbb{G}_{k, n-k}\right) \cong\left(\mathbb{E}_{k}^{n}\right)^{*} \otimes \mathbb{E}_{n-k}^{n} \\
& \text { et } \mathcal{P}_{l_{2}}: T\left(\mathbb{G}_{k, n-k}\right) \stackrel{\cong}{\longrightarrow}\left(\mathbb{E}_{n-k}^{n}\right)^{*} \otimes \mathbb{E}_{k}^{n}
\end{aligned}
$$

sont des isomorphismes de fibrés vectoriels. 
En particulier, pour tout $k$-plan $\xi$ de $\mathrm{R}^{\mathrm{n}}$, on a deux isomorphismes d'espaces vectoriels :

$$
\begin{aligned}
& \left.\left(Q_{\ell_{1}}\right)_{\xi}: T_{3}\left(\mathbb{G}_{k, n-k}\right) \stackrel{\simeq}{\longrightarrow}\right\}^{*} \underset{\mathbb{R}}{\otimes} \xi^{\perp} \\
& \left(Q_{l_{2}}\right)_{\xi}: T_{3}\left(\mathbb{G}_{k, n-k}\right) \stackrel{\simeq}{\longrightarrow}(\xi+)_{\mathbb{R}}^{*} \xi
\end{aligned}
$$

D - Géométrie des sous-variétés

Soit $v$ une variété de dimension $n$, et $U$ une sous-variété de dimension $k$ régulièrement plongée (plongement noté $f$ ). Soit $G$ un sousgroupe de Lie de $G L(n, \mathbb{R})$. Conservant les conventions du paragraphe $B$, on pose encore :

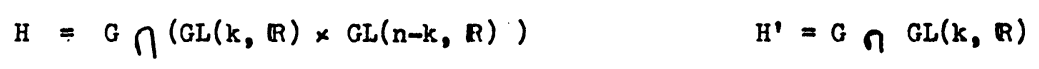

$H^{\prime \prime}=G \cap G L(n-k, R)$.

Soit $Q \rightarrow V$ (resp. $P^{\prime} \rightarrow U$ ) $I^{\prime e s p a c e ~ d e s ~ r e p e ̀ r e s ~ a d a p t e ́ s ~ a ̀ ~ u n e ~}$ G-structure sur $V$ (resp. à une $H^{\prime}$-structure sur $U$ ). On notera $\mathrm{r}_{U}(\mathrm{~V})$ au lieu de $T(V) \mid U$ I'espace des vecteurs tangents à $V$ ayant leur origine dans $U$. On appellera "fibré normal" tout sous-fibré vectoriel is de $T_{U}(V)$ tel que $T_{U}(V)=T(U) \oplus N$. Soit $N$ un tel fibré normal.

Soit $P_{N}$ le sous-ensemble de $\mathrm{Q} / \mathrm{U}$ formé des repères de $\mathrm{V}$ dont les $k$ premiers vecteurs sont tangents à $U$ et les $(n-k)$ derniers sont dans $N$. Supposant $\mathrm{P}_{\mathrm{N}} \cap(\mathrm{Q} \mid \mathrm{U})_{\mathrm{x}} \neq \varnothing \quad \forall \mathrm{x} \in \mathrm{U}, \mathrm{P}_{\mathrm{N}}$ est alors un H-sous-fibrésprincipal de $Q / U$. On dira alors que la $H^{\prime}$-structure $P^{\prime} \longrightarrow U$ 
est compatible avec la G-structure $Q \longrightarrow V$ relativement au fibré normal in, si $P^{\prime}$ est égal au $\mathrm{K}^{\prime}$-fibré principal $\left(\mathrm{P}_{\mathrm{H}}\right) / \mathrm{H}^{\prime \prime}$.

Supposons désormais cette compatibilité vérifiée; et soit P" le H"fibré principal $\left(\mathrm{P}_{N}\right) / \mathrm{H}^{\prime} \quad\left(\mathrm{P}_{\mathrm{N}}=\mathrm{P}^{\prime} \otimes \mathrm{P}^{\prime \prime}\right)$. Notons $\mathrm{p}_{\mathrm{U}}$ et $\mathrm{p}_{\mathrm{N}}$ les projections de $T_{U}(V)$ sur $T(U)$ et $N$ parallèlement à $\mathbb{N}$ et $T(U)$.

Soit $D$ la connexion linéaire sur $V$ associée à une G-connexion $\omega$ sur $Q$. Notons $D^{\prime}$ et $D^{\prime \prime}$ les projections de $D$ sur $T(U)$ et $N$, $Q_{l^{l}}$ et $Q_{l^{2}}$ les secondes formes fondamentales. La connexion $D^{\prime}$ est une connexion linéaire sur $U$, associée à une $H^{\prime}$ connexion $\omega^{\prime}$ sur $P^{\prime}$ d'après le théorème III. B. 3.

$\left.1^{\circ}\right)$ Torsions induites [resp. $\left.D^{\prime}\right]$.

Soit $\mathcal{G}$ (resp. $\left.C^{\prime}\right)$ la torsion $d_{D}(\text { id })_{v}\left[\right.$ resp. $\left.a_{D^{\prime}}(\text { id })_{U}\right]$ de $D$

\section{Proposition III. D. I}

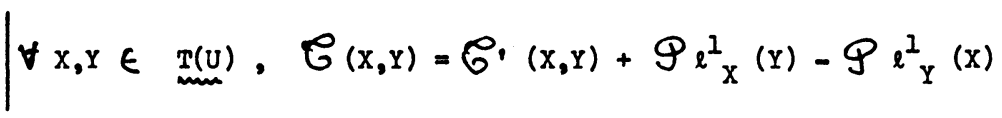

En effet, $G(X, Y)=D_{X} Y-D_{Y} X-[X, Y]$

$=p^{\prime}\left(D_{X} Y\right)-p^{\prime}\left(D_{Y} X\right)-[X, Y]+p^{\prime \prime} D_{X} Y-p^{\prime \prime} D_{Y} X$

$=\varrho^{\prime}(X, Y)+Q e_{X}^{I}(Y)-e_{Y}^{I}(X)$. 


\section{Corollaire III. D. 2}

Si $\mathscr{C}=0$, alors $\mathscr{C}$, est également nul et la forme bilinéaire $(X, Y) \longrightarrow\left(P_{\ell_{1}}\right)_{X}(Y)$ de $(T(U))^{2}$ dans $\mathbb{N}$ est symétrique.

\section{Corollaire III. D. 3}

Si la G-structure $Q$ est pseudo-intégrable ([l]) c'est-à-dire a un tenseur de structure nul, et $s^{\prime} i l$ existe un fibré normal $\mathbb{N}$ tel que $P^{\prime}$ soit compatible avec $Q$ relativement $\grave{a} N$, la $H^{\prime}$-structure $P^{\prime}$ est également pseudo-intégrable.

\section{$\left.2^{\circ}\right)$ Structures pseudo-riemanniennes}

Supposons que $G$ soit le groupe $O^{p}(n, \mathbb{R})$ des matrices inversibles de $G L(n, \mathbb{R})$ qui respectent la forme bilinéaire symétrique non dégénérée $\sum_{i}^{p} x_{i} y_{i} \sum_{i=p+1}^{n} x_{i} y_{i}$. La donnée d'une G-structure $Q$ sur $v$ est alors équivalente à la donnée d'une métrique pseudo-riemannienne $g$. Supposons en outre qu'en tout point $x$ de $U$, la forme bilinéaire symétrique $g^{\prime} x$ induite par $g_{x}$ sur le sous-espace $T_{x}(U)$ de $T_{x}(V)$ soit non dégénérée : l'orthogonal $\mathrm{N}_{x}$ de $T_{x}(U)$ dans $T_{x}(V)$ relativement à $E_{x}$ est alors un supplémentaire de $T_{x}(U)$ dans $T_{x}(V)$ et $I$ 'on définit ainsi un fibré normal orthogonal $\mathrm{N}=\bigsqcup_{\mathrm{x} \in \mathrm{U}} \mathrm{N}_{\mathrm{x}}$

Les propositions III. A. 3 et III. A. 4 sont alors appliquables. On a, en outre, le 
Théorème III. D. 4

Soit $D$ la connexion de LEVI-CIVITA de la variété pseudo-riemannienne $(V, g)$.

(I) la projection $D^{\prime}$ de $D$ sur $T(U)$ parallèlement à $N$ est la connexion de LEVI-CIVITA de la variété pseudo-riemannienne (U, $\left.B^{\prime}\right)$.

(II) la forme bilinéaire $(X, Y) \longrightarrow\left(Q_{\ell_{I}}\right)_{X}(Y)$ de $(T(U))^{2}$ dans $N$ est symétrique, et est la forme polaire de la seconde forme quadratique fondamentale de la théorie des sous-variétés d'une variété pseudo-riemannienne.

$D^{\prime a p r e ̀ s ~ I I I . ~ A . ~} 3, \quad D^{\prime} \mathrm{X}^{\mathrm{g}^{\prime}}=0 \quad \forall \mathrm{X} \in \underset{\mathrm{T}}{\mathrm{T}(\mathrm{U})}$

D'après III. D. $2, \quad B_{1}=0$

Or la connexion de LEVI-CIVITA de $\left(U, G^{\prime}\right)$ est l'unique connexion linéaire sur $U$, ̀̀ torsion nulle et vérifiant $D^{\prime} g^{\prime}=0$. Elle ne peut donc qu'être égale à $D^{\prime}, d^{\prime}$ où (I).

Puisque $E^{0}=0$, la forme $(X, Y) \longrightarrow\left(Q_{l_{1}}\right)_{X}(Y)$ est symétrique đ'après III.D.2), Elle est donc la forme polaire de la forme quadratique $\mathrm{X} \longrightarrow \mathcal{Q}_{\mathrm{X}}(\mathrm{X})=\mathrm{p}_{\mathrm{N}}\left(\mathrm{D}_{\mathrm{X}} \mathrm{X}\right)$ : on reconnâ̂t bien là la seconde forme quadratique fondamentale de la théorie des sous-variétés d'une variété pseudo-riemannienne.

La partie (II) de ce théorème justifie la terminologie adoptée pour $Q \ell_{1}$ et $Q \ell_{2}$. En outre, on a ici :

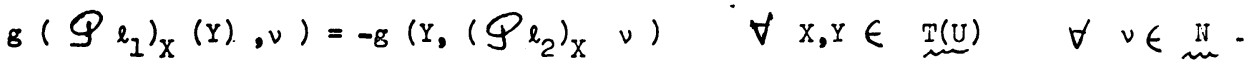


Lorsque $(V, g)$ est une variété localement euclidienne, Iesi equations

$$
\begin{cases}R^{\prime}+Q_{l_{2}} \wedge Q_{l_{1}} & =0 \\ d_{D} Q_{l_{1}} & =0 \\ R^{\prime \prime}+Q_{l_{1}} \wedge Q_{l_{2}} & =0 \\ d_{\hat{D}} Q_{l_{2}} & =0\end{cases}
$$

ne sont autres que les classiques équations de GAUSS-CODAZZI elles sont cquivalentes à I'équation $\Omega^{\prime}+\nabla_{\omega^{\prime}} \quad \mathrm{Pl}+\left[\begin{array}{ll}\mathrm{P} \ell, \mathrm{Pl} & 0\end{array}\right]=0$ de II. A. 4 , ou aux quatre projections de cette équation sur $\underline{H}^{\prime}, \underline{H^{\prime \prime}}, d_{1}$ et $d_{b_{2}}$. Si la codimension de $U$ dans $V$ est $I$, les deux dernières équations sont identiquement vérifiées.

Le théorème III. D. 4 permet de généraliser en termes de connexions linéaires, et indépendamment de toute métrique, la théorie classique des sousvariétés d'une variété pseudo-riemannienne ("théorie des courbes, et des surfaces" dans l'espace euclidien). Pour plus de détails, cf. [15] et [22] . 
Chapitre IV

Extensions triviales

d'une connexion
A - Extensions principales triviales
B - Extensions vectorielles triviales
C - Comparaison entre extensions triviales vectorielles et principales
D - Théorèmes d'existence dans le cas $G=O(n)$ 


\section{A - Extensions principales triviales d'une connexion}

Soit $\mathrm{H}$ un sous-groupe de Lie de $\mathrm{G}$, et th un supplémentaire de $\mathrm{H}$ dans $G$ tel que ad(H). Clb $C_{-}$db.

Si $\mathrm{P} \longrightarrow U$ est un H-fibré principal, on notera $\tilde{\mathrm{P}} \longrightarrow U$ le G-fibré principal obtenu à partir de $P$ par extension du groupe structural.

10) Définitions

Soit $P$ (resp. $P^{\prime}$ ) un H-fibré principal muni d'une connexion $\omega$ (resp. $\left.\omega^{\prime}\right)$. On appellera morphisme de $(P, \omega)$ dans $\left(P^{\prime}, \omega^{\prime}\right)$ tout morphisme $\varphi$ de H-fibrés principaux de $P$ dans $P^{\prime}$ tel que $\omega=\omega^{\prime} . \varphi$ (on définit ainsi une catégorie).

On appellera G-extension à courbure nulle d'une connexion $\omega$ sur un H-fibré principal $P$ une connexion $\tilde{\omega}$ à courbure nulle sur $P$, telle que $\quad \omega=(\tilde{\omega} \mid \mathrm{P})_{\underline{H}}$. Une telle G-extension à courbure nulle sera dite triviale si, en outre, le groupe d'holonomie de $\tilde{\omega}$ est 0 (1).

Si $\varphi$ est un morphisme de $(P, \omega)$ dans $\left(P^{\prime}, \omega^{\prime}\right)$, et si $\underset{\sim}{\tilde{\omega}}$ ' est une G-connexion à courbure nulle (resp. triviale) de $\omega^{\prime}, \tilde{\omega}^{\prime}, \tilde{\varphi}$ est une G-extension à courbure nulle (resp. triviale) de $\omega$ que l'on appellera l'image réciproque de $\tilde{\omega}^{\prime}$ par $\varphi[\tilde{\varphi}$ désignant le morphisme de G-ribrés principaux $\mathrm{P} \longrightarrow \mathrm{P}^{\prime}$ obtenu par extension de $\varphi$ ].

(1) Si U est simplement connexe, toute extension à courbure nulle est automatiquement triviale. 
$\left.2^{\circ}\right)$ Rôle universel de la connexion canonique de l'espace homogène réductif $G / H$

Soit $P_{0}$ le H-fibré principal $G \longrightarrow G / H$, et $\omega_{0}$ sa connexion canonique $\omega_{0}(d g)=\left(\bar{g}^{-} d g\right)_{\underline{H}}$. Nous avons déjà vu (II. C. exemple 4 ) que $\tilde{P}_{\circ}$ est le fibré trivial $G \times G_{/ H} \longrightarrow G_{/ H}$ et que la connexion $\tilde{\omega}_{0}$ sur $\tilde{P}_{0}$, définie par $\tilde{\omega}_{0}(d g, d x)=\bar{g}^{-1} d g$, est une G-extension triviale de $\omega_{0}$. Donc tout morphisme $\varphi$ de $(P, \omega)$ dans $\left(P_{0}, \omega_{0}\right)$ induit, par image réciproque de $\tilde{\omega}_{0}$, une G-extension triviale de $\omega$.

Réciproquement, toute G-extension triviale de $\omega$ peut être obtenue de cette façon : plus précisément, on a le

\section{Théorème IV. A. I}

(I) L'application qui, à tout morphisme $\varphi$ de $(P, \omega)$ dans $\left(P_{0}, \omega{ }_{0}\right)$, associe l'image réciproque de $\tilde{\omega}_{0}$ par $\mathcal{Y}$, est une application surjective de l'ensemble des morphismes de $(P, \omega)$ dans $\left(P_{0}, \omega_{0}\right)$ sur l'ensemble des G-extensions triviales de $\omega$.

(II)Si l'on suppose en outre la base $U$ de $P$ connexe, une condition nécessaire et suffisante pour que $\tilde{\omega}_{0}$ ait même image réciproque par deux morphismes $\Psi$ et $f^{\prime}$ de $(P, \omega)$ dans $\left(P_{0}, \omega_{0}\right)$, est qu'il existe un élément $g \in G$, tel que $\varphi^{\prime}=I_{g} \cdot \mathscr{Y}, L_{g}$ désignant la translation à gauche par $g$ dans $P_{0}=G$.

\footnotetext{
Soit $\tilde{\omega}: T(\tilde{P}) \rightarrow G$ une G-extension triviale de $\omega \cdot$ Puisque

$\stackrel{\sim}{P}$ est trivial et $\tilde{\omega}$ à holonomie nulle,il existe une fonction $\psi: \stackrel{\sim}{P} \longrightarrow G$

telle que $\quad \psi(\xi . E)=\psi(\xi) . \xi, \quad \forall \xi \in \stackrel{\sim}{P}, \forall \xi \in G$ $\tilde{\omega}=\psi^{-1} \cdot d \psi$.
} 
On en déduit que la restriction $\Psi$ de $\psi$ à $P$ est un morphisme de H-fibrés principaux de $P$ dans $P_{0}$, telle que $\psi=\left(\varphi^{-1} \cdot d \varphi\right)_{\underline{H}}$ puisque $\omega=(\tilde{\omega} / P)_{\underline{H}}$, d'où la partie $(I)$ du théorème.

Soient $\varphi$ et $\mathcal{L}^{\prime}$ deux morphismes de $P$ dans $P_{O}=G \rightarrow(G / H)$. Soient $\psi$ et $\psi^{\prime}$ les fonctions de $\tilde{P}$ dans $G$ obtenues en composant $\tilde{\Psi}$ et $\tilde{\varphi}^{\prime}$ avec la projection de $G \times G / H$ sur $G$. On a alors :

$$
\tilde{\omega}_{0} \cdot \tilde{\varphi}=\psi^{-1} \cdot d \psi \text { et } \quad \tilde{\omega}_{0} \cdot \tilde{\varphi}^{\prime}=\psi^{1-1} \cdot d \psi^{\prime}
$$

Dire que ces images réciproques sont les mêmes implique l'existence d'une fonction $B: \tilde{P} \longrightarrow G$ localement constante et constante sur chaque fibré telle que $\psi^{\prime}(\xi)=E(\xi) \cdot \psi(\xi) \quad \forall \xi \in \tilde{P}$. ( [10] p. 55 - proposition 2). Si donc $U$ est connexe, la fonction $B$ est constante, et il est clair que $\Psi^{\prime}=\mathrm{J}_{G} \cdot \Psi$, d'où la partie (II).

\section{$\left.3^{\circ}\right)$ Cas analytique}

Supposons le H-fibré principal $P \rightarrow U$ analytique réel, et la connexion $\omega$ sur $P$ analytique. Supposons en outre $\stackrel{\sim}{P}$ trivial.

Théorème IV. A. 2 (passage du local au global)

Pour qu'il existe une connexion analytique sur $\tilde{P}$, qui soit une G-extension triviale de $\omega$, il faut et il suffit que dans chaque composante connexe $U_{i}$ de $U$, il existe un ouvert non vide $U^{\prime}{ }_{i}$ tel que $\left(P \mid U_{i}^{\prime}, w\right)$ admette une G-extension analytique triviale.

Il est évident que la condition est nécessaire. Pour montrer qu'elle est suffisante, on peut se ramener au cas où $U$ est connexe en raisonnant de façon indépendante dans chaque composante connexe. 
Supposons donc $U$ connexe, et soit $U^{\prime}$ un ouvert de $U$ tel que $\left(P \mid U^{\prime}, \omega\right)$ admette une G-extension analytique triviale. D'après IV. $A .1$, il existe un morphisme $\varphi^{\prime}$ (nécessairement analytique) de $\left(P \mid U^{\prime}, \omega\right)$ dans

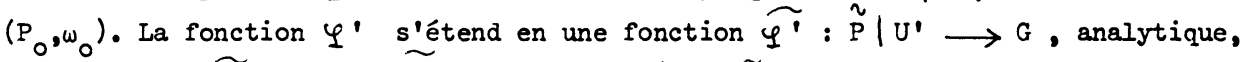
et vérifiant $\widetilde{\varphi^{\prime}}(\xi \cdot \varepsilon)=\widetilde{\varphi}^{\prime}(\xi), g \quad \forall \xi \in \widetilde{P} \mid U^{\prime}, \forall g \in$ G. Elle définit par conséquent une section analytique $\sigma^{\prime}$ ou $\stackrel{\sim}{P} \mid U^{\prime}$ (définie en prenant pour $\sigma^{\prime}(x)$ l'élément $\xi$ de $\left(\tilde{P} \mid U^{\prime}\right)_{x}$ tel que $\widetilde{\varphi^{\prime}}(\xi)$ soit l'élément neutre de $\left.G\right)$. Puisque $\stackrel{\sim}{P}$ est trivial, $\sigma^{\prime}$ s'étend en une section analytique $\sigma$ de $\stackrel{\sim}{P}$ tout entier (du moins si $U^{\prime}$ est connexe, ce qu'on peut toujours supposer), et permet par conséquent d'étendre $\widetilde{\varphi}^{\prime}$ en une fonction analytique $\widetilde{\Psi}: \stackrel{\sim}{P} \longrightarrow$ en posant :

$$
\xi=\sigma(p \xi) \cdot \tilde{\Psi}(\xi) \quad \forall \xi \in \widetilde{P}
$$

Puisque $\tilde{\Psi}(\xi \cdot g)=\tilde{\Psi}(\xi) \cdot g \quad \forall \xi \in \stackrel{\sim}{P} \quad \forall \xi \in G$, la 1-rorme $\tilde{\varphi}^{-1} \cdot d \tilde{\varphi}$ sur $\tilde{P}$ est une connexion analytique à courbure nulle, qui induit sur $P$ une connexion analytique $\left(\tilde{\varphi}^{-1} \cdot d \tilde{\mathscr{I}} / \mathrm{P}\right)_{\text {H }}$ qui coincide nécessairement avec $\omega$ sur $P \mid U^{\prime}$. Mais puisque $U$ est connexe, et puisqu'elles sont analytiques, ces deux connexions coincident partout, d'où le théorème.

B - Extensions vectorielles triviales d'une connexion

Reprenant désormais les conventions du paragraphe III-B, supposons $G$ inclus dans $G L(n, \mathbb{R})$ et posons $H^{\prime}=G \cap G L(k, R) \quad H^{\prime \prime}=G \cap G L(n-k, \mathbb{R})$ et $H=H^{\prime} \times H^{\prime \prime}=G \cap[G L(k, \mathbb{R}) \times G L(n-k, \mathbb{R})]$.

Pour toute variété $U$, on notera $\tilde{F}_{n}(U)$ le fibré vectoriel trivial $\mathbb{R}^{n} \times U \longrightarrow U$, et $\stackrel{\sim}{P}_{n}(U)$ le G-fibré principal associé $G \times U \longrightarrow U$. 


\section{$\left.1^{\circ}\right)$ Définitions}

Soit $P^{\prime} \longrightarrow U$ un $H^{\prime}$-fibré principal, et $E=\mathbb{R}_{\mathscr{R}_{k}}^{k}\left[\mathrm{P}^{\prime}\right]$ le fibré vectoriel modelé de fibre type $\mathbb{R}^{\mathrm{k}}$.

On appellera "plongement" de $E$ dans $\tilde{E}_{n}(U)$ tout homomorphisme injectif $\alpha: E \longrightarrow \tilde{E}_{n}(U)$, réalisant $E$ comme sous-fibré vectoriel de $\tilde{E}_{n}(U)$. On appellera "fibré normal" relatif à un plongement a tout sous-fibré vectoriel $N$ de $\tilde{E}_{n}(U)$ tel que $\tilde{E}_{n}(U)=\alpha(E) \oplus N$.

On notera $\mathrm{p}_{\alpha, \mathbb{N}}$ le morphisme surjectif $\tilde{E}_{n}(U) \rightarrow E$ obtenu en composant la projection de $\tilde{E}_{n}(U)$ sur $\alpha(E)$ parallèlement à If avec l'isomorphisme $\alpha^{-1}$ de $\alpha(E)$ sur $E$.

Pour tout fibré normal If relatif à $\alpha$, on notera $\mathrm{P}_{\mathrm{H}, \alpha}$ le sousensemble de $\tilde{P}_{n}(U)$ formé des repères $\xi$ de $\tilde{E}_{n}(U)$ dont les $k$ premiers vecteurs sont dans $\alpha(E)$ et les $(n-k)$ derniers dans $N$. Si $\mathrm{P}_{\mathrm{N}, \alpha} \cap\left(\tilde{\mathrm{P}}_{\mathrm{n}}(\mathrm{U})\right)_{\mathrm{x}} \neq \varnothing \quad \forall \mathrm{x} \in \mathrm{U}, \mathrm{P}_{\mathrm{N}, \alpha}$ est un H-sous-fibré principal différentiable de $\tilde{P}_{n}(U)$.

Un plongement $\alpha$ de $E$ (muni de la $H^{\prime}$-structure $P^{\prime}$ ) dans $\tilde{E}_{n}(U)$ (muni de la G-structure triviale $\tilde{P}_{n}(U)$ ) sera dit "adapté" relativement à N si

(1) $\mathrm{P}_{\mathrm{N}, \alpha}$ est un H-sous-fibré principal différentiable de $\tilde{P}_{\mathrm{n}}(\mathrm{U})$

(2) $\mathrm{P}^{\prime}$ est égal au H'-fibré principal $\mathrm{P}_{\mathrm{H}, \alpha} \mathrm{H}^{\prime \prime}$ 
Si $P^{\prime}$ est muni d'une connexion $\omega$, et $E$ de la connexion $D$ associée à $\omega$, on appellera n-extension triviale de $D$ un couple $(\alpha, N)$ formé d'un plongement $\alpha$ de $E$ dans $\tilde{E}_{n}(U)$ et d'un fibré normal $N$ relatif à $\alpha$ tels que

(1) a soit adapté relativement à $\mathrm{N}$

(2) la connexion $D$ corresponde par $\alpha$ à la connexion sur $\alpha(E)$ obtenue par projection parallèlement à $N$ de la connexion naturelle à courbure nulle du fibré vectoriel trivial $\tilde{E}_{n}(U)$.

Si $P^{\prime}{ }_{i} \longrightarrow U_{i}$ est un H-fibré principal $(i=1,2)$ muni d'une connexion $\omega_{i}$ et si le fibré vectoriel modelé $E_{i}=\mathbb{R}^{k}\left[\mathrm{P}^{\prime}{ }_{i}\right]$ est muni de la connexion $D$ associée $a ̀ \omega_{i}$, on appellera morphisme de $\left(E_{1}, D_{1}\right)$ dans $\left(E_{2}, D_{2}\right)$ tout morphisme strict de fibrés vectoriels $E \longrightarrow E^{\prime}$, associé à un morphisme de $\left(P_{1}^{\prime}, \omega_{1}\right)$ dans $\left(P_{2}^{\prime}, \omega_{2}\right)$.

Soit $\varphi$ un morphisme de $\left(E_{1}, D_{1}\right)$ dans $\left(E_{2}, D_{2}\right)$ au-dessus d'une application $\bar{\varphi}: \quad U_{1} \rightarrow U_{2}$ : Soit $\alpha_{2}$ un plongement de $E_{2}$ dans $\tilde{E}_{n}\left(U_{2}\right)$ : on définit un plongement $\alpha_{1}$ de $E_{1}$ dans $\tilde{E}_{n}\left(U_{1}\right)$ en posant $\left(\alpha_{1}\right)_{x}=\left(\alpha_{2}\right) \bar{\varphi}_{(x)} \cdot \varphi_{x}, \forall x \in U_{1} \cdot\left(\varphi_{x}\right.$ désigne l'isomorphisme $\left(E_{1}\right)_{x} \cong\left(E_{2}\right)_{\bar{\varphi}}(x)$ obtenu par restriction de $\varphi$ à $\left(E_{1}\right)_{x}$, et l'on a identifié de façon naturelle $\left(\tilde{E}_{n}\left(U_{1}\right)\right)_{x}$ et $\left(\tilde{E}_{n}\left(U_{2}\right)\right)_{\Psi}(x)$ à $\left.\mathbb{R}^{n}\right)$. On appellera $\alpha_{1}$ l'image réciproque de $\alpha_{2}$ par $\varphi$. Si $\mathbb{N}_{2}$ est un fibré normal relatif à $\alpha_{2}$, le fibré $N_{1}=N_{2} \cdot \bar{\varphi}$, obtenu comme image réciproque de $\mathrm{N}_{2}$ par $\varphi$, est un fibré normal relatif à $\alpha_{1}$. Si $\alpha_{2}$ est un plongement adapté de $E_{2} \underset{\sim}{\text { dans }} \tilde{E}_{n}\left(U_{2}\right)$ relativement à $N_{2}, \alpha_{1}$ est un plongement adapté de $E_{1}$ dans $E_{n}\left(U_{1}\right)$ relativement à $N_{1}$. Si $\left(\alpha_{2}, N_{2}\right)$ est une n-extension triviale de $D_{2},\left(\alpha_{1}, N_{1}\right)$ est une n-extension triviale de $D_{1}$. 
$\left.2^{\circ}\right)$ Rôle universel de la connexion canonique du fibré $\mathrm{E}_{\mathrm{k}}^{\mathrm{n}}$ modelé sur la variété de Stiefel généralisée

Reprenant les notations et définitions du paragraphe III-C, on a vu que $\tilde{E}_{n}\left(G_{k, n-k}\right)=E_{k}^{n} \oplus E_{n-k}^{n}$.

Soit $\alpha_{k}$ l'injection naturelle de $E_{k}^{n}$ dans $\tilde{E}_{n}\left(G_{k, n-k}\right)$ : c'est un plongement adapté de $E_{k}^{n}$ muni de la $H^{\prime}$-structure $v_{k, n-k}$ dans $\tilde{E}_{n}\left(c_{k, n-k}\right)$ relativement au fibré normal $E_{n-k}^{n}$. Par définition même de la connexion canonique $D_{k}$ sur $E_{n-k}^{n},\left(\alpha_{k}, E_{n-k}^{n}\right)$ est une $n$-extension triviale de $D_{k}$. Tout morphisme $\varphi$ de $(E, D)$ dans $\left(E_{k}^{n}, D_{k}\right)$ induit, par image réciproque de $\left(\alpha_{k}, E_{n-k}^{n}\right)$ une n-extension triviale de $(E, D)$. Réciproquement, toute n-extension triviale de $(E, D)$ peut être obtenue de cette façon : plus précisément; on a le

Théorème IV.B.I L'application qui, à tout morphisme $\varphi$ de $(E, D)$ dans $\left(E_{k}^{n}, D_{k}\right)$, associe I'image réciproque de $\left(\alpha_{k}, E_{n-k}^{n}\right)$ par $\Psi$, est une bijection de l'ensemble des morphismes de $(E, D)$ dans $\left(E_{k}^{n}, D_{k}\right)$ sur l'ensemble des $n$-extensions triviales de $D$.

Soit $(\alpha, N)$ une n-extension triviale de $D \cdot$. [E est supposé modelé sur $P^{\prime}$, et $D$ associé à une connexion $\omega$ sur $\left.P^{\prime}\right]$. Notons $\bar{\alpha}$ l'application $E \longrightarrow R^{n}$ obtenue en composant $\alpha$ avec la projection de $\tilde{E}_{n}(U)=\mathbb{R}^{n} \times$ sur $\mathbb{R}^{n}$. Soit $\bar{\varphi}$ l'application de $U$ dans $c_{k, n-k}$ qui, au point $x$ de $U$, associe le couple $\left(\bar{\alpha}\left(E_{x}\right), H_{x}\right) \in G_{k, n-k}$. Puisque $\left(E_{k}^{n}\right) \bar{\varphi}_{(x)}=\alpha\left(E_{x}\right)$, I'application 
$\Psi$ de $E$ dans $E_{k}^{n}$ dont la restriction à $E_{x}$ est l'isomorphisme $\alpha_{x}$ de $E_{x}$ sur $\alpha\left(E_{x}\right)$ est un morphisme strict de fibrés vectoriels de $E$ dans $E_{k}^{n}$, au-dessus de $\bar{\varphi}$.

Par construction de $\Psi$, l'image réciproque de $\left(a_{k}, \mathbb{E}_{n-k}^{n}\right)$ par $\Psi$ est égale à $(\alpha, \mathrm{II})$. Si $(\alpha, \mathbb{N})$ avait été obtenu comme image réciproque de $\left(\alpha_{k}, E_{n-k}^{n}\right)$ par un morphisme strict $\psi$ de $E$ dans $E_{k}^{n}$, on aurait en outre $\varphi=\psi$.

Puisque $(\alpha$, II $)$ doit être une n-extension triviale à la fois de $D$ et de la connexion $D_{k} \cdot \varphi$ image réciproque de $D_{k}$ par $\varphi$, ces deux connexions ne peuvent qu'être égales, et $\Psi$ est un morphisme de $(E, D)$ dans $\left(E_{k}^{n}, D_{k}\right)$ que $I^{\prime}$ on vérifie sans peine être associê à un morphisme de $\left(P^{\prime}, \omega\right)$ dans $\left(v_{k, n-k}, w_{k}\right)$.

Il est clair que l'application $(\alpha, \mathbb{N}) \rightarrow \varphi$ ainsi définie est l'application inverse de $\Psi \longrightarrow$ image réciproque de $\left(\alpha_{k}, E_{n-k}^{n}\right)$ par $\mathscr{\Psi}$, d'où le théorème.

\section{$\left.3^{\circ}\right)$ Cas d'une connexion linéaire}

Si $P^{\prime} \rightarrow U$ est un espace de repères sur une variété $U$ de dimension $k$, le fibré modelé $E=\mathbb{R}^{k}\left[P^{\prime}\right]$ est alors le fibré tangent $T(U)$, et toute connexion $D$ sur $E$ est une connexion linéaire. 
a) Proposition IV. B. 2

Pour toute n-extension triviale $(\alpha, N)$ de $D$, la torsion $\xi_{0}$ de $D$ est donnée par la formule :

$$
\sigma=p_{\alpha, N} \cdot d \bar{\alpha}
$$

[où à désigne la l-forme sur $U$ à valeurs dans $\mathbb{R}^{n}$, obtenue en composant $\alpha: T(U) \longrightarrow \mathbb{R}^{\mathrm{n}} \times U$ avec la projection de $\mathbb{R}^{\mathrm{n}} \times U$ sur $\left.\mathbb{R}^{\mathrm{n}}\right]$.

En effet $E_{(X, Y)}=D_{X} Y-D_{Y} X-[X, Y] \quad \forall X, Y \in \underset{m}{T(U)}$.

or $\quad D_{X} Y=p_{\alpha, N}(X \cdot \bar{\alpha}(Y))$

$D_{Y} X=p_{\alpha, N}(Y \cdot \bar{\alpha}(X))$

$\left.[\mathrm{X}, \mathrm{Y}]=\mathrm{p}_{\alpha, \mathrm{N}}(\dot{\alpha}[\mathrm{X}, \mathrm{Y}])\right)$

et $\quad X \cdot \bar{\alpha}(Y)-Y \cdot \bar{\alpha}(X)-\bar{\alpha} \cdot([X, Y])=d \bar{\alpha}(X, Y) \quad$ c.q.f.d.

(on a identifié $\mathbb{R}^{n}$ et $\left(\tilde{E}_{n}(U)\right)_{x}$ en tout point $x$ de $U$ ).

b) Soit $f$ une immersion de $U$ dans la variété $\mathbb{R}^{\mathrm{n}}$.

La différentielle de définit un plongement $\alpha_{f}$ de $T(U)$ dans $\tilde{E}_{n}(U)$ (vérifiant df $=\bar{\alpha}_{f}$ où $\bar{\alpha}_{f}$ est obtenue en composant $\alpha_{f}$ avec la projection de $\tilde{E}_{n}(U)$ sur $\left.R^{n}\right)$.

Supposons le plongement $\alpha_{f}$ de $T(U)$ dans $\widetilde{E}_{n}(U)$ adapté ( $T(U)$ étant muni de la $H^{\prime}$-structure $P^{\prime}$ ) relativement à un fibré normal $N^{\circ}$ On dira alors que : $f$ est une immersion adaptée de $U$ (munie de la H'-structure $\left.P^{\prime}\right)$ dans $\mathbb{R}^{\mathrm{n}}$ (munie de sa G-structure plate canonique). 
Si $f$ est une telle immersion adaptée, on lui associe une application : $\bar{\varphi}_{f}: U \longrightarrow G_{k, n-k}$ en associant au point $x$ de $U$ le couple (df $\left.\left(T_{x}(U)\right), N_{x}\right) \in G_{k, n-k}$ (comme dans la démonstration du théorème IV. B. I) et un morphisme strict $\varphi_{f}$ de $T(U)$ dans $E_{k}^{n}$, au-dessus de $\bar{\varphi}_{f}$. (On appelle $\bar{\varphi}_{\mathrm{f}} \quad \mathrm{l}$ 'application de GAUSS associée à $\mathrm{f}$ ).

\section{Proposition IV. B. 3}

L'image réciproque de la connexion canonique $D_{k}$ par l'application de GAUSS $\bar{\varphi}_{f}: U \longrightarrow G_{k, n-k}$ est une connexion linéaire à torsion nulle, adaptée à la $\mathrm{H}^{\prime}$-structure $\mathrm{P}^{\prime}$ sur $\mathrm{U}$.

Il est clair que $\Psi_{f}$ est associée à un morphisme de H-fibrés principaux : $P^{\prime} \longrightarrow v_{k, n-k}$, et que par conséquent, $l^{\prime}$ image réciproque $D=D_{k} \cdot \varphi_{f}$ de $D_{k}$ par $\Psi_{f}$ est adaptée à la $H^{\prime}$-structure $P^{\prime}$.

Par construction de l'application de GAUSS, $\left(\alpha_{f}, \mathbb{N}\right)$ est l'image réciproque de $\left(\alpha_{k}, E_{n-k}^{n}\right)$ par $\varphi_{f}$. Appliquant alors la proposition IV. B. 2,

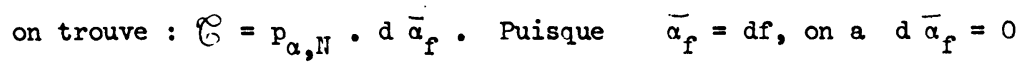
d'où $\mathscr{C}=0$.

Cas particulier $G=O(n) \quad H^{\prime}=O(k)$

La donnée $d^{\prime} u n e H^{\prime}$-structure $P^{\prime}$ sur $U$ est équivalente à la donnée $d^{\prime}$ une métrique riemannienne $g$ sur $U$. Dire qu'une immersion $f: U \longrightarrow \mathbb{R}^{n}$ est adaptée relativement à un fibré normal $N$ signifie que $f$ est une isométrie de $(U, E)$ dans l'espace euclidien $\mathbb{R}^{n}$, le fibré normal $N$ ne pouvant être alors que le supplémentaire orthogonal de $d f\left(T(U)\right.$ ) dans $\tilde{E}_{n}(U)$. 
Comme il n'existe qu'une seule connexion linéaire $D_{0}$ sur $U$ qui soit à la fois sans torsion et adaptée à la structure riemannienne $B$ (la connexion de LEVI-CIVITA), la proposition IV. B. 3 fournit le résultat suivant, dû à Sh. KOBAYASHI ( [9] ) :

\section{Proposition IV. B. 4}

Soit $f$ une immersion isométrique d'une variété riemannienne $U$ de dimension $k$ dans l'espace euclidien $\mathbb{R}^{n}$. Notant $D_{0}$ la connexion de LEVI-CIVITA de $U$, l'application de GAUSS $\varphi_{f}$ associée à $f$ est un morphisme de $\left(T(U), D_{0}\right)$ dans $\left(E_{k}^{n}, D_{k}\right)$.

C - Comparaison entre extensions triviales vectorielles et principales

Soit toujours $G$ un sous-groupe de lie de $G L(n, \mathbb{R})$, $H^{\prime}=G \cap G L(k, \mathbb{R}), H^{\prime \prime}=G \cap G L(n-k, \mathbb{R})$, $H=H^{\prime} \times H^{\prime \prime}=G \cap[G L(k, R) \times G L(n-k, R)]$. Soit db le supplémentaire de $\underline{H}$ dans $G$ défini au paragraphe III. $B$, et notons $d P$ le supplémentaire $d b \oplus \underline{H^{\prime \prime}}$ de $\underline{H}^{\prime}$ dans $\underline{G}$ (vérifiant $\operatorname{ad}\left(H^{\prime}\right) d^{P} \subset d$ ).

Soit $\mathrm{P}^{\prime} \longrightarrow U$ un $\mathrm{H}^{\prime}$-fibré principal. Munissant le fibré vectoriel modelé $E=\mathbb{R}^{k}\left[P^{\prime}\right]$ de la connexion $D$ associée à une connexion $\omega$ sur $P^{\prime}$, on se propose de comparer les deux propriétés suivantes :

- il existe une n-extension (vectorielle) triviale de $D$

- il existe une G-extension (principale) triviale de $\omega$. 
$D^{\prime}$ après les théorèmes IV. A. $I$ et IV. B. $I$, on est ramené à étudier l'existence de morphismes entre $\left(v_{k, n-k}, \omega_{k}\right)$ et $\left(P_{0}, \omega_{0}\right)$ où $v_{k, n-k}$ et $P_{0}$ désignent les $\mathrm{H}^{\prime}$-fibrés principaux $G / \mathrm{H}^{\prime \prime} \longrightarrow G / \mathrm{H}^{\prime} \times \mathrm{H}^{\prime \prime}$ et $\mathrm{G} \longrightarrow \mathrm{G}_{/ \mathrm{H}^{\prime}}$ munis de leur connexion canonique.

Lemme IV. C. I

Le morphisme naturel $G \stackrel{F}{\longrightarrow} G / H^{\prime \prime}$ de $P_{0}$ dans $V_{k, n-k}$<smiles>[H][R1]([H])([H])C(C)(C)CC</smiles>

est un morphisme de $\left(P_{0}, w_{0}\right)$ dans $\left(v_{k, n-k}, w_{k}\right)$.

Ce résultat s'obtient immédiatement à partir des définitions de $\omega_{0}$ et $\omega_{\mathrm{k}}$.

\section{Lemme IV. C. 2}

Pour tout point $\left(\xi, \xi^{\prime}\right)$ de $G_{k, n-k}=G / H^{\prime} \times H^{\prime \prime}$, il existe un voisinage $G_{\left(\xi, \xi^{\prime}\right)}$ de $\left(\xi, \xi^{\prime}\right)$ dans $G_{k, n-k}$ et un morphisme $\left(v_{k, n-k} \mid G\left(\xi, \xi^{\prime}\right)\right) \longrightarrow G \quad$ de

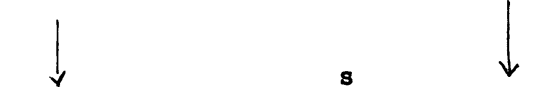

$G\left(\xi, \xi^{\prime}\right)$ G/H'

$\left(v_{k, n-k} G\left(\xi, \xi^{\prime}\right), w_{k}\right)$ dans $\left(P_{0}, w_{0}\right)$. 
Géométriquement, $F$ est l'application qui, à tout repère $E \in G$ de $\mathbb{R}^{\mathrm{n}}$, associe le couple formé du k-repère de $\mathbb{R}^{\mathrm{n}}$ engendré par les $\mathrm{k}$ premiers vecteurs de $B$, et du $n-k$ plan supplémentaire engendré par les $n-k$ derniers vecteurs de $\mathrm{g}$.

L'application $f$, qui associe au couple formé d'un k-plan et d'un $n-k$ repère supplémentaire le couple formé de ce $k$-plan et du $n-k$ plan engendré par le $(n-k)$ repère, est la projection naturelle du II"-fibré principal $v_{n-k, k} \longrightarrow G_{k, n-k}$

Soit $\left(\xi, \xi^{\prime}\right)$ un point de $G_{k, n-k}, G_{\left(\xi, \xi^{\prime}\right)}$ un voisinage ouvert de ce point au-dessus duquel $v_{n-k, k} \longrightarrow G_{k, n-k}$ est trivial, et soit $s$ : $G_{\left(\xi, \xi^{\prime}\right) \longrightarrow V_{n-k, k}}=G_{/ H^{\prime}}$ une section de ce fibré au-dessus de $G_{\left(\xi, \xi^{\prime}\right)} \cdot$ Soit $S$ l'application $\left(v_{k, n-k} / G_{\left(\xi, \xi^{\prime}\right)}\right) \rightarrow$ G qui, à tout couple $\left(z, \xi_{l}^{\prime}\right)$ formé d'un k-repère $z$ et d'un $(n-k)$ plan supplémentaire $\xi_{1}^{\prime}$, associe le n-repère de $R^{n}$ obtenu en complétant $z$ par le $(n-k)$ repère $s\left(\left(\xi_{1}, \xi_{1}^{\prime}\right)\right)$ (où $\xi_{1}$ désigne le k-plan engendré par $z$ ).

Il est clair que $(S, s)$ est un morphisme de $\mathrm{H}^{\prime}$-fibrés principaux ; et, puisque $F \circ S$ est l'injection naturelle de $\left(\left.v_{k, n-k}\right|_{\left(\xi, \xi^{\prime}\right)}\right)$ dans $v_{k, n-k}$ et que $\omega_{k} \cdot F=\omega_{0}$ (lemme IV. C. 1), $\omega_{0} \cdot S$ est la restriction de $\omega_{k} \grave{a}$ $\left(\left.v_{k, n-\underline{k}}\right|^{G}\left(\xi, \xi^{\prime}\right)\right)$ Le lemme IV. C. 2 en résulte. 
Des propriétés universelles de $\omega_{0}$ et $\omega_{k}$, on déduit le

\section{Corollaire IV. C. 3}

(I) L'existence d'une G-extension principale triviale de $\omega$ [équivalente à $I^{\prime}$ existence $d^{\prime}$ un morphisme de $\left(P^{\prime}, \omega\right)$ dans $\left.\left(P_{0}, \omega_{0}\right)\right]$ entrâ̂ne l'existence $d^{\prime} u n e$ n-extension triviale de $D$ [équivalente à l'existence d'un morphisme de $\left(P^{\prime}, \omega\right)$ dans $\left.\left(v_{k, n-k}, w_{k}\right)\right]$.

(II) La réciproque n'est vraie que localement : s'il existe une n-extension vectorielle triviale de $D$, tout point $x$ de $U$ admet un voisinage $U_{x}$ tel que $\left(P \mid U_{X}, \omega\right)$ admette une G-extension principale triviale.

Les fibrés $P_{0}$ et $V_{k, n-k}$ sont, en fait, analytiques ainsi que les connexions $\omega_{0}$ et $w_{k}$. Les morphismes $(S, s)$ peuvent être choisis analytiques. Du théorème IV. A. 2 et du corollaire IV. C. 3 , on déduit le

\section{Corollaire IV. C. 4}

$\mathrm{Si} \mathrm{P}^{\prime}$ et $\omega$ sont analytiques, et si le G-fibré principal $\mathrm{P}^{\prime}$ (obtenu à partir de $P^{\prime}$ par extension du groupe structural) est trivial, l'existence d'une G-extension principale triviale analytique de $\omega$ est équivalente à l'existence d'une n-extension vectorielle triviale analytique de $D$.

\section{$D$ - Théorèmes d'existence dans le cas $G=O(n)$}

Soit $P^{\prime} \longrightarrow U$ un $O(k)$-fibré principal différentiable dont la base $U$ est de dimension $\leqslant p$, et soit $D$ la connexion sur $E=\mathbb{R}^{k}\left[\mathrm{P}^{\prime}\right]$ associée à une connexion $\omega$ sur $P^{\prime}$. 
IARASIMHAN et RAIMAIIAI ont montré dans $[19]$ :

$\left.1^{\circ}\right)$ Il existe ${ }^{(1)}$ un morphisme différentiable $\psi$ de $(P, \omega)$ dans $\left(\mathbb{V}_{k, n-k}, \omega_{k}\right)$ si $n \geqslant 2 k^{3}(p+1)(2 p+1)$.

$2^{\circ)}$ Pour tout point $x$ de $U$, il existe un voisinage $U_{x}$ de $x$ dans $U$ et un morphisme différentiable $\Phi_{x}$ de $\left(P \mid U_{x}, \omega\right)$ dans $\left(\mathbb{V}_{k, n-k}, \omega\right)$ si $n \geqslant 2 k^{3}(2 p+1)$.

$\left.3^{\circ}\right) \mathrm{Si} \mathrm{P}^{\prime}$ et $\omega$ sont analytiques, les morphismes $\Phi_{x}$ précédents peuvent être choisis analytiques (2).

On en déduit le

\section{Théorème IV. D. 1}

(I) Il existe une $n$-extension vectorielle triviale de $D$

$$
\forall n \geqslant 2 k^{3}(p+1)(2 p+1)
$$

(II) Pour tout point $x$ de $U$, il existe un voisinage $U_{x}$ de $x$ dans $U$, tel que la restriction de $D$ à $E / U_{x}$ admette une n-extension vectorielle triviale $\forall \mathrm{n} \geqslant 2 \mathrm{r}^{3}(2 \mathrm{p}+1)$.

(III) $S i P^{\prime}$ et $\omega$ sont analytiques; et si $\tilde{P}^{\prime}$ est trivial, il existe une $\mathrm{n}$-extension vectorielle triviale analytique de $D$, avec $n=2 k^{3}(2 p+1)$.

(1) C'est pourquoi dans le cas $G=O(n)$, la connexion canonique $\omega_{k}$ sur $\mathbb{V}_{k, n-k}$ est appelée "connexion universelle".

(2) Il n'en est pas de même pour $\psi$, que l'on obtient en recollant les $\Phi_{x}$ à l'aide d'une partition différentiable de l'unité. 


\section{Corollaire IV. D. 2}

On en déduit le résultat d'analyse suivant :

Soit $\alpha: T(U) \longrightarrow \underline{O(k)}$ une l-forme à valeurs dans $\underline{O(k)}$. Supposons $U$ de dimension $\leqslant p$.

(I) Il existe alors, pour tout point $x$ de $U$, un voisinage $U_{x}$ de $x$ dans $U$ et une fonction différentiable $f: U_{x} \rightarrow O(n)$ (avec $n=2 k^{3}(2 p+1)$ ) telle que $\alpha \mid U_{x}=\left(\bar{f}^{-1} d f\right) \underline{O(k)}$ projection de $\underline{O(n)}$ sur $\underline{O(k)}$ parallèlement à $d^{p}=d b \oplus \quad \underline{O(n-k)}$.

(II) Si l'on suppose en outre $U$ et $\alpha$ analytiques, on peut choisir la fonction $f$ analytique, et la définir sur $U$ tout entier.

Il suffit, en effet, pour démontrer le corollaire, d'appliquer IV. D. I au cas où $P^{\prime}$ est le fibré trivial $O(k) \times U \rightarrow U$, et où $\omega$ est la forme de connexion dont l'image réciproque par la section canonique de $P^{\prime}$ est $\alpha$.

Au lieu d'appliquer les propriétés universelles à l'existence d'extensions triviales, on peut inversement abaisser le nombre $n$ de [19] en prouvant directement l'existence de n-extensions triviales :

Etude du cas $k=2$ :

Localement, on peut toujours supposer $E$ triviel : soit $\left(\sigma_{1}, \sigma_{2}\right)$ une base orthonormée de $E$. Puisque la dérivée covariante de la métrique doit être nulle, la connexion $D$ sur $E$ est définie par une l-forme à valeurs réelles $\gamma: T(U) \longrightarrow R$ en posant $D_{X} \sigma_{1}=\gamma(X) \cdot \sigma_{2}$ et $D_{X} \sigma_{2}=-\gamma(X) \cdot \sigma_{1}$ 


\section{Lemme IV. D. 3}

Si $\gamma$ admet un facteur intégrant, $D$ admet une 3-extension vectorielle triviale. Considérons en effet le plongement adapté $\alpha$ de $E$ dans $\widetilde{E}_{3}(U)$ défini par

$$
\begin{aligned}
& \alpha\left(\sigma_{1}\right)=r \cos \theta \cdot e_{1}+r \sin \theta \cdot e_{2}+\sqrt{1-r^{2}} \cdot e_{3} \\
& \alpha\left(\sigma_{2}\right)=-\sin \theta \cdot e_{1}+\cos \theta \cdot e_{2}
\end{aligned}
$$

où $e_{1}, e_{2}, e_{3}$ désignent les sections canoniques de $\widetilde{E}_{3}(U)$, et où $r$ et $\theta$ sont des fonctions arbitraires $U \longrightarrow \mathbb{R}$.

Prenant obligatoirement pour IN le fibré orthogonal à $\alpha(E)$ dans $\tilde{E}_{3}(U), P_{N, \alpha}$ est alors égal à la transposée $t_{\alpha}$ de $\alpha$; et par conséquent, la matrice

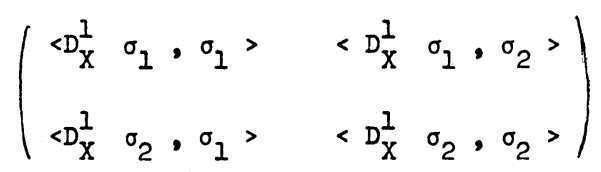

définissant la connexion $D^{l}$ sur $E$ obtenue par projection de la connexion naturelle à courbure nulle de $\widetilde{E}_{3}(U)$, est égale à

$$
\left(\begin{array}{cc}
r \cos \theta & r \sin \theta \\
-\sin \theta & \cos \theta
\end{array}\right) \cdot\left(\begin{array}{l}
\sqrt{1-r^{2}} \\
0
\end{array}\right) \cdot\left(\begin{array}{cc}
(X r) \cos \theta-(X \theta) r \sin \theta & -(X \theta) \cos \theta \\
(X r) \sin \theta+(X \theta) r \cos \theta & -(X \theta) \sin \theta \\
-(X r) \frac{r}{\sqrt{1-r^{2}}} & 0
\end{array}\right)
$$




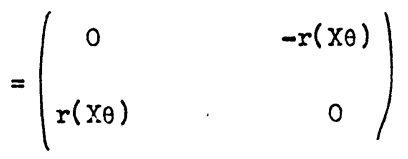

Donc, pour que $D^{I}=D$, il faut et il suffit que $\gamma=-r d \theta$. On pourra donc choisir $r$ et $\theta$ de façon que $\gamma=-r$ d $\theta$ dès l'instant que $\gamma$ admet un facteur intégrant.

\section{Corollaire IV. D. 4}

Soit $U$ une variété de dimension $2, P^{\prime} \rightarrow U$ un $O(2)$-fibré principal et $\omega$ une connexion sur $P^{\prime}$.

Il existe alors un morphisme de $\left(P^{\prime}, \omega\right)$ dans $\left(v_{k, n-k}, \omega_{k}\right)$

(I) - localement, avec $\mathrm{n}=3$

(II) - globalement, avec $\mathrm{n}=9$

(III) - globalement, avec $n=3$ si $\left(P^{\prime}, \omega\right)$ est analytique et si $\stackrel{\sim}{P^{\prime}}$ est trivial.

Toute 1-forme $\gamma$ sur une variété de dimension 2 admet localement un facteur intégrant.

Suivant un procédé standard, on peut passer d'un résultat local à un résultat global à condition de multiplier $n$ par $p+1$ (si $\operatorname{dim} U \leqslant p$ ) (cf. [19]), d'où (II).

La partie (III) résulte de IV. C. 4. 


\section{Remarques}

1) avec $k=2, p=r$ les nombres $n$ de NARASIMHAN et RAMANAN sont

$80=2 k^{3}(2 p+1)$ dans le cas local et $240=80(p+1)$ dans le cas global. On les a donc améliorés.

2) On généralise ainsi le fait que, localement, toute variété riemannienne à deux dimensions peut être isométriquement plongée dans $R^{3}$.

\section{Cas d'une variété riemannienne}

D'après le résultat de JANNET [8] cf. aussi $E$. CARTAN [2], on sait qu'il est possible de plonger isométriquement (localement) toute variété rieman$\underline{k(k+1)}$

nienne $U$ de dimension $k$ dans l'espace euclidien $\frac{R}{2}$.

On en déduit la

\section{Proposition IV. D. 5}

Soit $\mathrm{P}^{\prime} \longrightarrow \mathrm{U}$ le fibré des repères orthonormés adaptés à une structure riemannienne sur une variété $U$ de dimension $k$. Soit $\omega$ sa connexion de LLEVI-CIVITA.

$$
\begin{aligned}
& \text { II existe alors un morphisme de }\left(P^{\prime}, \omega\right) \text { dans }\left(v_{k, n-k}, \omega_{k}\right) \\
& \text { - localement, avec } n=\frac{k(k+1)}{2} \\
& \text { - globalement, avec } n=\frac{k(k+1)^{2}}{2} \\
& \text { - globalement, avec } n=\frac{k(k+1)}{2} \quad \text { si } \quad\left\{\begin{array}{l}
\left(P^{\prime} \mid U\right) \text { est analytique } \\
\tilde{P^{\prime}} \text { est trivial }
\end{array}\right.
\end{aligned}
$$


Remarque:

Avec $k=p$, les nombres $n$ de IIARASIMHAN et RAMANAN sont

$2 k^{3}(2 k+1)$ dans le cas local, et $2 k^{3}(2 k+1)(k+1)$. 
[1] D. BERNARD - Sur la Géométrie différentielle des G-structures Thèse (Paris) - Annales de l'Institut Fourier tome 10 (1960)

[2] E. CARTAN - Leçons sur la Géométrie des espaces de Riemann (Gauthier-Villars - Paris 1946)

[3] " - Oeuvres complètes - partie III - volume 2 - p. 1091

[4] Ch. EHRESMANN - Connexions de Cartan (Colloque de Eruxelles 1950)

[5] GOETZ - A general scheme of inducing infinitesimal connections

[6] " - Special connections associated with a given linear connection (Bu. Ac. pol. sc. - Naths - volume $10-n^{0} 1$ p. 29 et $n^{\circ} 5 \mathrm{p} .277$ )

[7] V. GUILLEMAIN - On G-structure of finite type - Thèse (Harvard 1961)

[8] M. JANnET - Annales Soc. Polon. mathém. 5- 1926 - p. 35

[9] Sh. KOBAYASHI - Induced connections and imbedd:d riemannian spaces (Nazoya Math. Jour. vol. $10-1956$ - p. 15)

[10] J.L. KOSZUL - Fibre Pundles and differential Geometry - (Institute of fundamental Research - Bombay - 1960) 


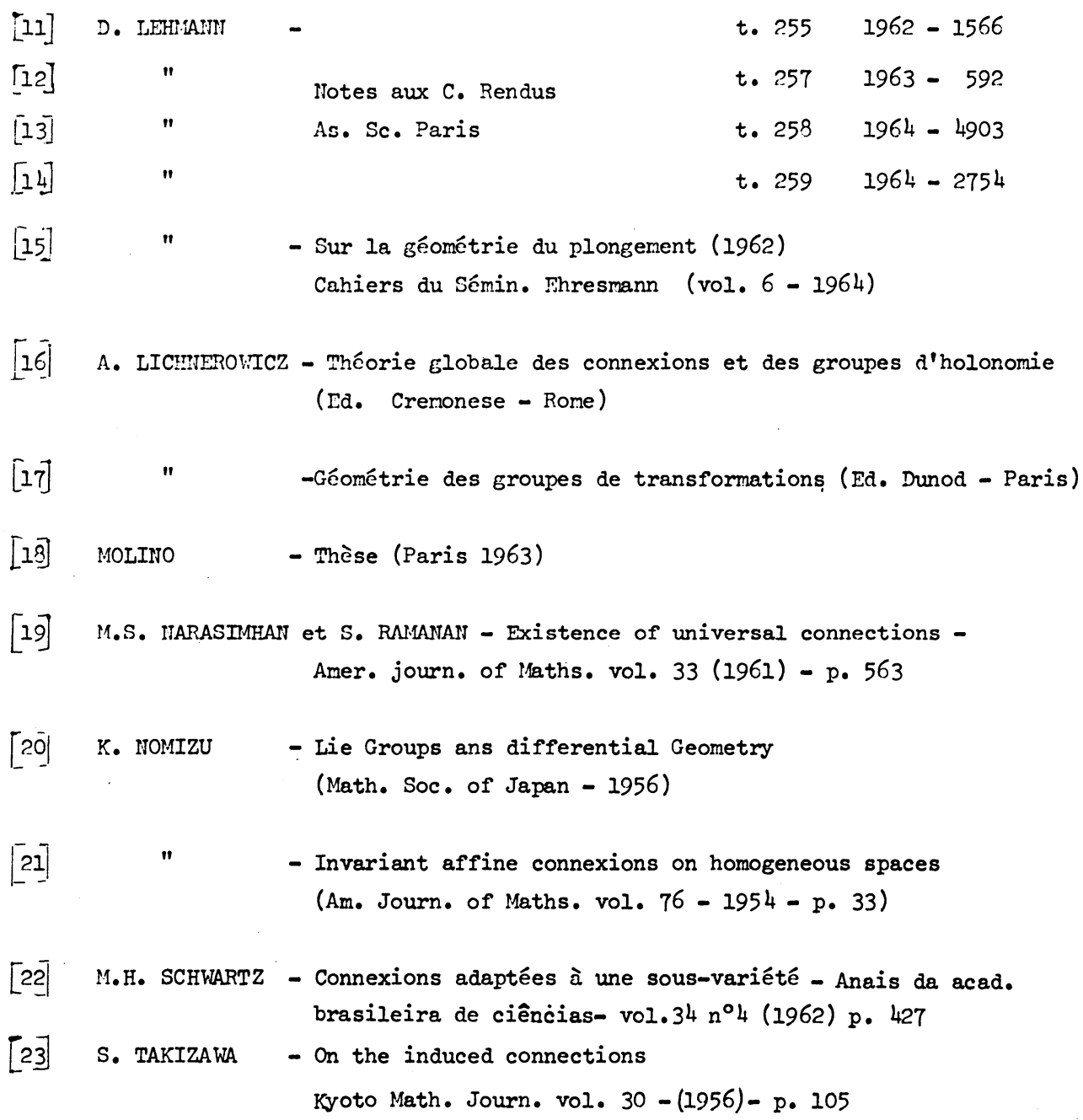



Ies fibrés à groupe structural variable en Géométrie d'ordre supérieur 
Introduction

Il nous a semblé utile de rassembler et comparer différentes définitions des connexions d'ordre supérieur.

On sait que définir une connexion sur un fibré principal équivaut à réduire le groupe structural d'un certain prolongement de ce fibré. Ainsi, KOBAYASHI [7] a montré que la donnée d'une connexion linéaire sans torsion sur une variété $V^{~ r e v i e n t ~ a ̀ ~ m u n i r ~ l e ~ f i b r e ́ ~} H^{\perp}(V)$ des repères d'ordre $I$ de $V$ d'une structure de sous-fibré principal du fibré $H^{2}(V)$ des repères holonomes d'ordre 2 de $V$ (résultat étendu par P. LIBERMANN aux connexions linéaires avec torsion, à condition de remplacer $\mathrm{H}^{2}(V)$ par le fibré $\overline{\mathrm{H}}^{2}(\mathrm{~V})$ des repères semiholonomes d'ordre 2 de $V$ ). Tel est aussi le point de vue adopté par BOURBAKI dans $[1]$.

Toutefois, pour généraliser cette méthode et lui donner une pleine efficacité, il apparaît nécessaire d'admettre des espaces fibrés dont le groupe structural "varie" avec le point de base : c'est essentiellement ce que nous avons voulu montrer dans cet article, passant entièrement sous silence certains aspects plus géométriques des connexions (tels le transport parallèle infinitésimal ou global, et les dérivations covariantes).

Le chapitre I est consacréà des généralités sur les fibrés à groupe structural variable (notion dûe à H. CARTANT [2], reprise par J. FREMKELL [5]). En plus de généralisations immédiates de propriétés classiques des fibrés à groupe structural fixe, on montre (th. 1 et 2) qu'un fibré à groupe structural variable peut aussi s'interpréter comme un fibré à groupe structural fixe (groupe "tordu"). (Le théorème 1 ci-dessous admet come corollaire le th. du par. II.I de FREIKEL ( $\left.[5], n^{\circ} 15, p .169\right)$. D'autre part, une construction analogue à celle de notre théorère $I$ est faite dans [I] à propos du prolongement ${ }^{t} \Omega \longrightarrow{ }^{\infty} \mathrm{V}$ d'un fibré principal $\Omega \rightarrow \mathrm{V}$ dans le cas "non trivial"). 
Utilisant alors l'outil ainsi introduit, on redéfinit dans la seconde partie les connexions holonomes d'ordre $m$ au sens d'EHRESMANn [4] (et l'on peut considérer le théorème 6 comme une généralisation à l'orare supérieur et au cas où $Q$ n'est pas nécessairement un espace de repères, du résultat de KOBAYASYI rappelé précédemment). On montre d'autre part (théorème 7).qu'une telle connexion induit naturellement une A-connexion au sens de ROURBAYI [l] pour toute algèbre locale $A$ de rang $\geqslant m$, et que rćciproquement toute $\mathbb{R}_{1}^{m}$-connexion peut être obtenue ainsi d'une façon et d'une seule (où $\mathbb{R}_{I}^{\mathrm{m}}$ désigne $I^{\prime}$ algèbre locale des séries formelles à $I$ indéterminée, tronquées à l'ordre $m+1$ ). On redémontre aussi (théorème 8) un résultat de HGO VAN QUE [9] exprimant qu'une connexion holonome au sens d'EHRESMAMN sur un fibré principal $Q$ induit naturellement une sur connexion holonome au sens de P. IIBERMANN [8] sur tout fibré vectoriel $\mathrm{E}$ associé à $Q$, et que réciproquement toute surconnexion peut être obtenue ainsi d'une façon et d'une seule lorsque $\Omega_{2}$ est l'ensemble de tous les isomorphismes d'espaces vectoriels de la fibre type sur une fibre de $E$.

Dans la partie III, on réexpose (toujours à l'aide des fibrés à groupe structural variable) la notion de $t$-connexion (BOURBAYI [I]), où cto désigne un espace fibré en algèbres locales.

Le sujet est loin d'être recouvert ici. En particulier, nous n'avons pas mentionné la généralisation au cas semi-holonome, lequel semble constituer en fait le domaine naturel de la Géométrie d'ordre supérieur. Il y aurait d'autre part beaucoup à dire du cas particulier des sous-fibrés principaux d'un espace de repères d'ordre arbitraire ("stṛuctures infinitésimales" suivant la terminologie d'EHRES!IANI) : les prolongements de ces fibrés peuvent exceptionnellement être considérés comme des fibrés principaux à groupe structural fixe ; on y définit torsion et géodésiques; et la torsion des connexions d'orare supérieur peut être utilisée pour redéfinir les obstructions successives à la platitude d'une structure infinitésimale (GUILLFAIN, STERNBERG [6] et STERNBERG, SINGER [10] ), généralisant le procédé utilisé par BINRIARD pour définir le premier tenseur de structure (P. LIBERIAMIN et JIGO VAII OUT nous ont signalé avoir des textes en préparation à ce sujet). 
Cet article fait suite à la lecture d'un papier inédit de 10 . BOURBAKI (les "Pierres"), qu'il a bien voulu nons communiquer.

Nous $I^{\prime}$ en remercions vivement. 
. 


\section{I - Espaces fibrés admettant un "espace structural fibré en groupe"}

Conventions

Soient $V$ et $F$ deux variétés différentiables ${ }^{(1)}$. On notera $\mathcal{F}_{\mathrm{V}}$ (resp. $\Psi_{\mathrm{V}}^{\mathrm{F}}$ ) la catégorie dont les objets sont les espaces fibrés différentiables localement triviaux de base $V$ (resp. et de fibre type $F$ ), et dont les morphismes sont les applications différentiables se projetant sur l'application identité de V (resp. et qui sont "stricts", c'est-à-dire induisent des difféomorphismes de fibre sur fibre).

Pour tout fibré $E$ et tout point $x$ de la base $V$ de $E, E_{x}$ désignera la fibre en $x$ de $E$.

Si $E$ (resp. $\left.F^{\prime}\right)$ est un objet de $\mathcal{F}_{V}^{F}$ (resp. $\left.\mathcal{F}_{V}^{F_{V}^{\prime}}\right)$; on notera $E E^{\prime}$ le produit fibré $\bigcup_{x \in V}\left(E_{x} \times E_{x}^{\prime}\right)$ de $E$ et $E^{\prime}$ : c'est un objet de $G_{V}^{F \times}$

Pour tout fibré principal $Q \longrightarrow V$ de groupe structural $G$ (de Lie), et pour toute variété $M$ sur laquelle $G$ opère différentiablement à gauche par un homomorphisme de groupes $Q: G \rightarrow \operatorname{Aut}(M)$, on notera ${ }^{M} Q(G)[Q]$ (ou $M_{G}[Q]$ s'il n'y a pas d'ambigüité sur $Q$ ) le fibré de fibre type $M$ et de base $V$, modelé sur $Q$ à l'aide de la représentation $\mathbb{R}$.

(1) "différentiable" signifiera toujours : de classe $c^{\infty}$. 


\section{A - Espaces fibrés presque principaux}

Soient $G$ et $L$ deux groupes de Lie, et $Q: I \rightarrow$ Aut $(G)$ un homomorphisme de groupes de Lie. Soit $R \longrightarrow V$ un fibré principal différentiable de groupe structural I . (On écrira parfois en abrégé $\lambda(g)$ au lieu de $Q(\lambda) \cdot g)$.

Le fibré modelé $\mathcal{Y}=G_{Q(L)}[R]$ possède, en tout point $x$ de $V$, une fibre $\xi_{x}$ canoniquement munie d'une structure de groupe de Lie isomorphe (non canoniquement) à $G$.

\section{Définition 1}

Soit $Q \longrightarrow V$ un objet de $G_{V}^{G}$.

On dira que l'on a muni $Q$ d'une structure de "fibré presque principal" d'espace structural $\xi_{\text {, }}$, si I'on s'est donné un morphisme $\Phi$ de la catégorie $\bar{F}_{v}$ $\Phi: \imath \otimes g \longrightarrow Q$ tel que, pour tout point $x$ de $v, \ell_{x}$ opère

à droite sur $\dot{Q}_{x}$ de façon simplement transitive par l'application $\Phi_{x}: \gamma_{x} \times \psi_{y_{x}} \longrightarrow \eta_{x}$ déduite de $\Phi$. groupe est définie par :

$$
\text { Soit } G \underset{S}{x} \text { L le produit semi-direct de } G \text { et } L \text {, où la loi de }
$$

$$
(\gamma, \lambda)\left(\gamma^{\prime}, \lambda^{\prime}\right)=\left(\gamma \cdot Q(\lambda) \gamma^{\prime}, \lambda \lambda^{\prime}\right) \quad \forall \gamma, \gamma^{\prime} \in G, \quad \forall \lambda, \lambda^{\prime} \in L
$$

et soit $\Omega \longrightarrow V$ un fibré presque principal d'espace structural $g=G G(L)[R]$. 


\section{Théorème 1}

(i) $Q \otimes R \longrightarrow V$ possède une structure naturelle de fibré principal différentiable de groupe structural $G \underset{Q}{X} L, G \underset{Q}{x} L$ opérant à droite $\operatorname{sur} Q \otimes R$ de façon suivante :

$$
\begin{aligned}
(q, r)(\gamma, \lambda)=(q \cdot r(\gamma), r \cdot \lambda) \quad & \forall(q, r) \in(q \otimes R)_{x} \\
& \forall(r, \lambda) \in G{\underset{Q}{x}}^{x} I
\end{aligned}
$$

$\left(r(\gamma)\right.$ désignant $l$ 'image de $\gamma$ par l'isomorphisme $\left.r: G \stackrel{\underline{\underline{z}}}{\rightarrow} \mathcal{G}_{x}\right)$.

(ii) Dans la catégorie $G_{\mathrm{V}}^{\mathrm{G}}, \Omega$ est canoniquement isomorphe au fibré modelé

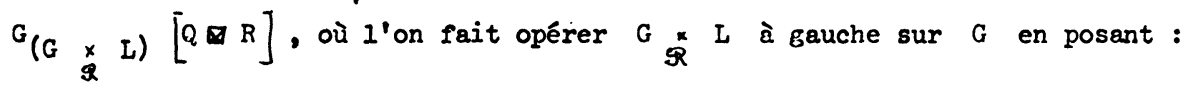

$$
<(\gamma, \lambda), B>=\gamma \cdot R(\lambda) G \quad \forall(\gamma, \lambda) \in G \mathbb{Q}_{R}^{x} L, \quad \forall G \in G
$$

On définit un isomorphisme de ${ }^{G}(G \times L)\left[\begin{array}{lll}Q & \otimes R\end{array}\right]$ sur $Q$ en associant, à la classe d'équivalence $\overline{(c,(q, r)})$ de $(\tilde{E},(q, r))$ modulo $G_{\mathbb{R}}^{x} L$, l'élément $q \cdot r(g)$ de $O$. Cet élément $q \cdot r(G)$ ne dépend pas du renrésentant $(g,(q, r))$ choisi dans la classe d'équivalence $\overline{(g, \overline{(q, r)})}$ : tout autre élément de cette classe d'équivalence est en effet de la forme :

$$
\left(<(\gamma, \lambda)^{-1}, E>,(q, r)(\gamma, \lambda)\right)
$$

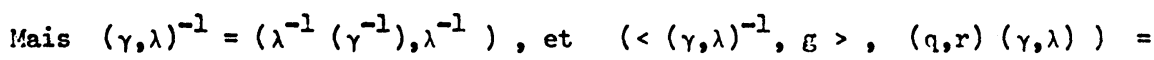

$$
\left(\lambda^{-1}\left(r^{-1}\right) \cdot \lambda^{-1}(g),(q \cdot r(\gamma), r \lambda)\right)=\left(g^{\prime},\left(q^{\prime}, r^{\prime}\right)\right)
$$


avec $g^{\prime}=\lambda^{-1}\left(r^{-1} g\right), q^{\prime}=q \cdot r(\gamma), r^{\prime}=r \lambda$.

On a donc $q^{\prime} \cdot r^{\prime}\left(g^{\prime}\right)=q r(\gamma) \cdot r \lambda \lambda^{-1}\left(\gamma^{-1} g\right)=q r(\gamma) \cdot r\left(\gamma^{-1} g\right)$

$$
=q \cdot r\left(\gamma \gamma^{-1} g\right)=q \cdot r(E)
$$

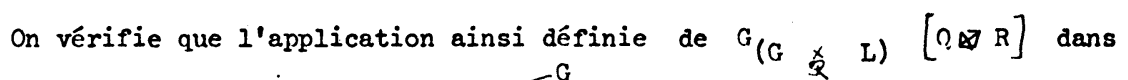
$Q$ est bien un isomorphisme dans $F_{\mathrm{V}}^{\mathrm{G}}$, d'où le théorème.

\section{Remarques :}

$\left.1^{\circ}\right)$ Par cet isomorphisme, $G_{x}$ opc̀re à droite sur $\left(G_{G} \times L[q \otimes R]\right)_{x}$ de la façon suivante :

$\overline{(g,(q, r))} \cdot \varepsilon_{x}=\overline{\left(g \cdot r^{-1}\left(E_{x}\right),(q, r)\right.}$

$\forall \overline{(\varepsilon,(q, r)}) \in\left(G_{G}{ }_{g}^{x} L[q \otimes R]\right)_{x}, \quad \forall \varepsilon_{x} \in l_{j x}$.

$\left.2^{\circ}\right)$ Si $L$ est le sous-groupe de $\operatorname{Aut}(G)$ réduit à $\left\{I_{G}\right\}, ~ d_{f}$ est le fibré trivial $G \times V \longrightarrow V$, et $G=G \underset{\mathscr{R}}{\times} L$. On a $O \otimes R=Q$ et un fibré presque principal d'espace structural $\xi_{f}$ n'est alors rien d'autre qu'un fibré principal de Eroupe structural G .

B - Fibrés modelés sur un fibré presque principal

Soit $\Omega \longrightarrow v$ un fibré presque principal d'espace structural $\mathcal{f}$. Soit alt $\longrightarrow V$ un fibré localement trivial de fibrc type $M$, et soit 
$\rho: y \otimes d t \longrightarrow d t$ un morphisme de la catégorie $T_{1}$ tel que, pour tout point $x$ de $v, \xi_{x}$ opère à gauche sur dt $x$ par l'application $e_{x}: y_{x} \times d l_{x} \rightarrow c t_{x}$ déduite de $e$.

$$
\text { Notons } d_{x}=d b_{x} \times \frac{a_{x}}{g_{x}} \text { I'espace quotient de } d b_{x} \times a_{x} \text { par }
$$

la relation d'équivalence $(m, q) \sim\left(r^{-1} m, q r\right)$

$$
\begin{gathered}
\forall(m, q) \in d_{x} \times Q_{x} \\
\forall r \in \varphi_{x}
\end{gathered}
$$

\section{Définition 2}

L'ensemble $d P=\bigcup_{x \in V} d P_{x}$ admet une structure naturelle de fibré difrérentiable localement trivial de base $V$ et fibre type $M$. On l'appellera le fibré de fibré-type cth, modelé sur $Q$ par la représentation $p$, et on le notera $d=d f_{\rho(g)}[Q]$ (ou $d b_{g}[Q]$ s'il n'y a pas d'ambigüité sur $e$ ).

\section{Cas particulier (K)}

Supposons
a) que $\xi=G \mathscr{R}(L)[R]$
b) que $c$ th $=M_{f(L)}[R]$
c) que $G$ opère différentiablement à gauche sur $M$ par une application $(\gamma, m) \longrightarrow\langle\gamma, m\rangle$ de $G * M$ dans $M$ telle que $\lambda\langle\gamma, m\rangle=\langle\lambda(\gamma), \lambda(m)\rangle$.

On peut alors faire opérer $\dot{y}_{\mathrm{x}}$ à gauche sur $\mathcal{M}_{\mathrm{x}}$ par une application $\varepsilon_{x}:\left(\varepsilon_{x}, m_{x}\right) \longrightarrow r\left\langle r^{-1}\left(g_{x}\right), r^{-1}\left(m_{x}\right)\right\rangle$ (avec $\left.g_{x} \in g_{x}, m_{x} \in d b_{x}, r \in R_{x}\right)$ il est clair que cette définition ne dépend pas de l'élément $r$ choisi dans $R_{x}$. On a alors : 


\section{Théorème 2:}

(i) G $\underset{Q}{x} L$ opère à gauche sur $M$ par l'application $((\gamma, \lambda), m) \longrightarrow<\gamma, \lambda(m)>$.

(ii) Les fibrés $d P=d b_{g}[Q]$ et ${ }^{M}(G \quad x \quad L)[Q \otimes R]$ sont canoniquement isomorphes dans la catégorie $\mathscr{G}_{\mathrm{V}}^{\mathrm{U}}$.

On définit un isomorphisme de $\left.M_{(G}{ }_{R} L\right)[0 \otimes R]$ sur $d b_{y}[Q]$ en associant, à la

classe d'équivalence $\overline{(m,(q, r)}$ de $(m,(q, r))$ modulo $G$ ${ }_{Q}^{x}$ L, l'élément $\overline{\overline{(r(m), q)}}$ de $\mathcal{P}_{x}((x=$ projection de $(q, r))$ égal à la classe d'équivalence de $(r(m), q)$ modulo $\mathcal{G}_{x}$. Cet élément de $d_{x}$ ne dépend pas du représentant $(m,(q, r))$ choisi dans la classe d'équivalence $\overline{(m,(q, r)})$ : tout autre élément de cette classe est en effet de la forme $\left((\gamma, \lambda)^{-1}(m),(q, r)(\gamma, \lambda)\right)$.

Or $(\gamma, \lambda)^{-1}(m)=\left(\lambda^{-1}\left(\gamma^{-1}\right), \lambda^{-1}\right)(m)=\left\langle\lambda^{-1}\left(\gamma^{-1}\right), \lambda^{-1}(m)>=\lambda^{-1}<\gamma^{-1}, m>\right.$

Et $(q, r)(\gamma, \lambda)=(q \cdot r(\gamma), r \lambda)$. Posons

$m^{\prime}=\lambda^{-1}<\gamma^{-1}, m>, q^{\prime}=q \cdot r(\gamma), r^{\prime}=r \lambda$.

On a alors $\left(r^{\prime}\left(m^{\prime}\right), q^{\prime}\right)=\left(r\left(\lambda \lambda^{-1}<\gamma^{-1}, m>\right), \operatorname{qr}(\gamma)\right)$

$$
=\left(r<\gamma^{-1}, m>, q \cdot r(\gamma)\right)=\left(\left\langle r\left(\gamma^{-1}\right), r(m)>, q \cdot r(\gamma)\right)\right.
$$

et $\left.\overline{\overline{\left(r^{\prime}\left(m^{\prime}\right), q^{\prime}\right)}}=\overline{\left(\langle r(\gamma))^{-1}, r(m)>, q \cdot r(\gamma)\right.}\right)=\overline{(r(m), q)}$. 


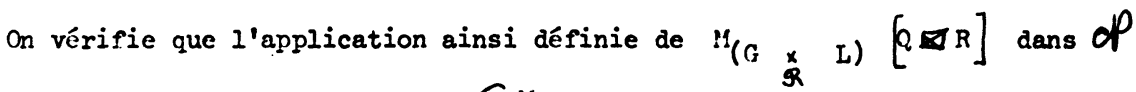
est bien un isomorphisme dans $G_{N} \frac{M}{V}$, d'où le théorc̀me.

Remarque :

Si $L=\left\{I d_{G}\right\},(\xi$ est alors trivial et $Q$ principal $), d b$ est le fibré trivial $\| \times \mathrm{V} \longrightarrow \mathrm{V}$. Dans ce cas, $\mathcal{P} \mathrm{n}$ 'est autre que le fibré modelé $M_{G}[?]$.

Revenons au cas général :

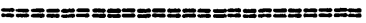

\section{Theorème 3}

Il existe une correspondance biunivoque canonique entre les sections différentiables de $\mathcal{A}=d_{b}$ y $[i]$ et les morphismes $\mathscr{f}$ de $?$ dans $d b$ (au sens de la catégorie $\Phi_{V}$ ) qui vérifient :

$$
\varphi\left(q \cdot g_{x}\right)=\left(g_{x}\right)^{-1} \cdot \varphi(q) \quad \forall g_{x} \in G_{x}, \quad \forall q \in Z_{x}
$$

A un tel morphisme $\varphi$, on associe la section $\sigma: v \longrightarrow d P$ qui, à tout point $x$ de $V$, associe la classe d'équivalence $\overline{(\overline{(q), q)} \text { de }}$ $\left(\xi(q), q\right.$ ) modulo $\xi_{x}$ (cette classe d'équivalence est indépendante de l'élément $q$ choisi dans $\left.\Omega_{x}\right)$. 


\section{C - Sous-fibrés presque principaux}

Soit $y=G Q(L)[R]$ un fibré en groupes de Lie. Soit II un sousgroupe de Lie de $G$ invariant par $R$, c'est-à-dire vérifiant $Q(L) . H=H$. On en déduit un fibré en groupes de Lie $H=H S(L)[R]$, qui est un sous-fibré en groupes de $\mathcal{G}$ en le sens évident suivant : il existe un morphisme $q$ (au sens de la catégorie $\Psi_{v}$ ) de $y_{b}$ dans $G$ tel que $\eta_{x}$ identifie $\mathscr{H}_{x}$ à un sous-groupe de Lie de $\xi_{x}$ en chaque point $x$ de $v$.

Soit $Q \longrightarrow V$ (resp. $P \longrightarrow V$ ) un fibré presque principal d'espace structural $\mathcal{G}$ (resp. Ho).

\section{Définition 3}

On dira que l'on a muni $P$ d'une structure de sous-fibré presque principal de $Q$, si l'on s'est donné un morphisme $i$ (de la catégorie $F_{V}$ ) de $P$ dans $Q$, vérifiant :

$$
i\left(q \cdot h_{x}\right)=i(q) \cdot Z\left(h_{x}\right) \quad \forall x \in V, \forall q \in P_{x}, \quad \forall h_{x} \in \mathscr{E}_{x}
$$

$\left(i\right.$ definit en particulier une injection de chaque fibre $P_{x}$ dans la fibre $i_{x}$ correspondante).

De façon habituelle, $G$ opère à gauche sur G/H par l'application $(Y, \tilde{B}) \longrightarrow \widetilde{Y \cdot G}$ (où $\tilde{\mathbb{B}}$ désigne, pour tout élément $G$ de $G$, la classe à droite nodulo $\mathrm{H}$ de $(\mathrm{C})$.

D'autre part, puisque $y$ est invariant par la représentation $\mathbb{R}$ de $I$ dans $G, R$ se factorise et permet de faire opérer $L$ à gauche sur $G / H$ par l'application :

$$
(\lambda, \widetilde{B}) \longrightarrow \widetilde{\lambda(E)} \quad \forall \lambda \in L \quad \forall \widetilde{E} \in G / H
$$


Puisque $\lambda(\gamma) \cdot \lambda(\tilde{g})=\overline{\lambda(\gamma) \cdot \lambda(g)}=\widetilde{\lambda(\gamma g)}=\lambda \cdot \gamma \widetilde{E}$

on est dans le cas particulier $(x)$ du paragraphe $B$; en particulier, $\mathcal{G}_{x}$ opère à gauche sur $\left(G_{/ H}(L)[R]\right)_{x}$ - On a alors le :

\section{Théorème 4}

Les sous-fibrés presque principaux de $\Omega$, admettant $y b$ comme espace structural, sont en correspondance biunivoque canonique avec les sections différentiables de $(y / y)$ \& $[?]$.

Soit en effet $P$ un tel sous-fibré presque principal : pour tout point $q$ de $\eta_{x}$. notons $\tilde{\sigma}(q)$ la classe à droite modulo $\gamma_{x}$ d'un quelconque élément $g_{x}$ de $\zeta_{x}$ tel que $q \cdot g_{x} \in P_{x} \quad(\tilde{\sigma}(q)$ ainsi défini ne dépend pas de l'élément

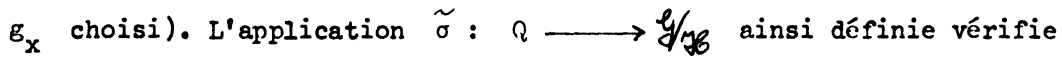
$\tilde{\sigma}\left(q \cdot r_{x}\right)=\left(r_{x}\right)^{-1}$. $\tilde{\sigma}(q)$ et définit par conséquent une section différentiable $\sigma$ de $\left(y / H_{b}\right) \xi[0]$, en vertu du théorème 3 .

Réciproquement, soit $\sigma$ une section de ce fibré définissant une application $\tilde{\sigma}: \Omega \longrightarrow \xi / \gamma_{0}$. Soit $P_{x}$ l'ensemble des éléments $q$ de $\beta_{x}$ tels que $\tilde{\sigma}(q)$ soit égal à la classe à droite modulo $\mathfrak{H}_{x}$ de l'élément neutre de $G_{x}:$ l'ensemble $P=\bigcup_{x \in V} P_{x}$ possède une structure naturelle de sous-fibré presque principal de $\Omega$, admettant the come espace structural. Les deux applications définies sont inverses l'une de l'autre, d'où le théorème. 
II - Prolongements holonomes des espaces fibrés

Connexions holonomes d'ordre superieur

Rappels sur la terminologie des jets et points proches (cf. $[1],[3]$ et $[8]$ )

On appellera (1) algèbre locale de rang in toute algèbre associative et comnutative $A$ de dimension finie sur le corps $\mathbb{R}$ des nombres réels, dont aucun élément $n^{\prime}$ est inversible, telle que $A^{m} \neq 0$ et $A^{m+1}=0$. Soit $\mathscr{L}^{m}$ la catégorie dont les objets sont les algèbres locales de rang $m$, et les mor-

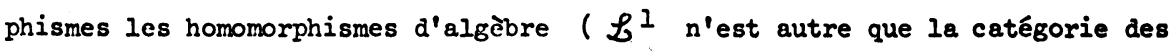
espaces vectoriels réels de dimension finie). Pour tout couple d'algèbres $A$ et $B$, on notera $H(\Lambda, B)$ l'ensemble des homomorphismes d'algèbre de $A$ dans $B$.

Soit $V$ une variété $C^{\infty}$ et $D(V)$ l'algèbre des fonctions $C^{\infty}$ de $V$ dans $\mathbb{R}$. Pour tout point $x$ de $V$ et tout entier $m \geqslant 1, I_{x}(V)$ désignera l'algèbre des germes en $x$ d'applications $\varphi \in D(V)$ nulles en $x$, et $I_{x}^{m}(v)$ l'algèbre locale de rang $m$ égale à $I_{x}(V) /\left(I_{x}(V)\right)^{m+1}$

Pour toute algèbre locale $\Lambda$ de rang $m$, on appelle A-point de $V$ proche de $x$ tout élément de $\left.\mathrm{H}_{\left(\mathrm{I}^{\mathrm{m}}\right.}^{\mathrm{m}}(\mathrm{V}), A\right)$.

(1) Cette définition est un peu différente de celle de [1I]: ce qu'on appelle ici algc̀bre locale n'est que l'idéal maximal d'une algèbre locale au sens de A. WEIL (WEIL considère les homomorphismes d'algèbres à élément unité. Mais il existe évidemment une équivalence naturelle entre les deux catégories ainsi définies). 
Soient $V$ une autre variété, $f$ une application différentiable de $V$ dans $\because, x$ un point de $V$ et $y=f(x)$ son image par $f$. L'application transposée $f^{*}: D(v) \longrightarrow D(V)$ induit par passares aux quotients une apPlication $j_{x}^{m} f \in H\left(I_{y}^{m}(V), I_{x}^{m}(V)\right)$ appelée le m-jet de $f$ en $x$. Les points $x$ et $y=f(x)$ sont appelés respectivement la "source" et le "but" du jet.

On a réciproquement le :

Lerme I

Pour tout élément $u \in I !\left(I_{y}^{m}(V), I_{x}^{m}(v)\right)$, il existe une application différentiable $f: V \rightarrow V$ telle que $j_{x}^{m} f=u$.

On posera $J_{x y}^{m}(v, v)=n i\left(I_{y}^{m}(v), I_{x}^{m}(v)\right)$.

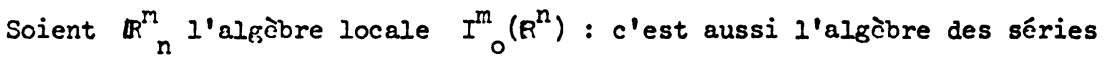
formelles, à $n$ indéterminées, sans terme constant, tronquées à l'ordre $m+1$. On appelle vecteur tangent $\bar{a} \mathrm{~V}$ en $x$, d'ordre $\mathrm{g}$ et de dimension $n$ tout élément de $J_{\text {ox }}^{m}\left(R^{n}, V\right)=H\left(I_{x}^{m}(v), \mathbb{R}_{n}^{m}\right)$, c'est-à-dire tout $\mathbb{R}_{n}^{m}$ - point de $v$ proche de $x$.

$$
\text { L'application } f_{*} \text { qui, à toute application } \gamma \text { de } \mathbb{R}^{n} \text { dans } V \text {, as- }
$$
socie l'application composée $f \cdot \gamma$ de $\mathbb{R}^{\mathrm{n}}$ dans $V$, induit une application

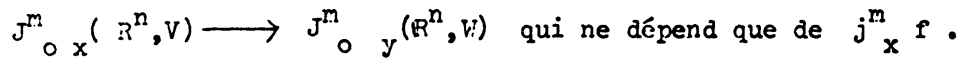

On a réciproquement le 


\section{Lemme 2}

Pour $n=1$, pour que deux applications $f$ et $f^{\prime}$ de $V$ dans $V$ induisent la même application $J^{m} x^{(\mathbb{R}, V)} \longrightarrow J_{0}^{m}(R, V)$, il faut et il suffit qu'elles aient même m-jet en $x$.

On pourra donc identifier le jet $j_{x}^{m} f$ à l'application $J^{m} \circ x^{(R, V)} \rightarrow J^{m} \circ y(R, W)$ qu'il définit.

Si $n$ est la dimension de $V$, les algèbres locales $\mathbb{R}_{n}^{m}$ et $I_{x}^{m}(V)$ sont isomorphes (non canoniquement) : un isomorphisme $z: R_{n}^{m} \cong I_{x}^{m}$ (V) $s^{\prime}$ appelle un m-repère de $V$ en $x$. L'ensemble $\mathrm{H}^{\mathrm{m}}(\mathrm{V})$ des m-repères de $\mathrm{V}$ possède une structure naturelle de fibré principal de base $V$ et groupe structural le groupe $I_{n}^{m}$ des automorphismes d'algèbre de $R_{n}^{m}$. La fibre en $x$ du fibré modelé $\left(\mathbb{R}_{n}^{\mathrm{m}}\right)\left(\mathrm{I}_{n}^{\mathrm{m}}\right)\left[\mathrm{H}^{\mathrm{m}}(\mathrm{V})\right]$ s'identifie naturellement à ${ }^{\mathrm{m}}{ }_{x}(\mathrm{~V})$. Pour toute algèbre locale $A$ de rang $m, I_{n}^{m}$ opòre naturellement à gauche sur $H\left(\mathbb{R}_{n}^{m}, A\right)$ et la fibre en $x \quad\left(A_{V}\right)_{x}$ du fibré modelé $A_{V}=H\left(R_{n}^{m}, A\right)_{\left(L_{n}^{m}\right)}\left[H^{m}(V)\right]$ est l'ensemble des A-points de $V$ proches de $x$.

Toute alEèbre locale $A$ de rang $m$ s'identifie de façon naturelle à $H\left(R_{1}^{m}, A\right)$. En particulier, $I_{x}^{m}(V)$ s'identifie à $J_{x}^{m}(V, \mathbb{R})$. 
Pour tout fibré $E \longrightarrow V$, on notera $J^{m} E \longrightarrow V$ le fibré dont la fibre $\left(\mathrm{J}^{\mathrm{m}}\right)_{x}$ en un point $x$ de $V$ est $r^{\prime}$ ensemble des m-jets $j_{x}^{m} s$ des sections locales $s$ de $E$ définies au voisinage de $x$; on appelle $\mathrm{J}_{\mathrm{E}}$ le prolongement holonome d'ordre $m$ de $E$.

A - Prolongement holonome d'ordre m d'un fibré principal

Soit $Q \rightarrow V$ un fibré principal de groupe structural $\Gamma$. Posons $n=\operatorname{dim} v$, et soit $G^{m}={ }^{\left(R_{n}^{m}\right)}$ r le groupe de Lie des $\mathbb{R}_{n}^{m}$ - points de $\Gamma$. Le groupe $L^{m}$ opère à gauche sur $G^{m}$, et le fibré modelé $\left.G^{m}=G^{m}{ }_{\left(I^{m}\right.}^{m}\right)^{m} H^{m}(V)$ admet comme fibre en $x$ le groupe de Lie ${ }_{x}^{I_{x}^{m}(V)} \Gamma$ des m-jets de source $x$ d'applications de $\mathrm{V}$ dans $\Gamma$.

$$
\text { Posons } j_{x}^{m} \cdot j_{x^{m}}^{m}=j_{x}^{m}(s g) \quad \forall j_{x}^{m} \in\left(J^{m}\right)_{x} \text { et }
$$

$\forall j_{x}^{m} B \in\left(y^{m}\right)_{x} \quad$ (ou sg représente la section locale $x \longrightarrow s(x) \cdot g(x)$ de २): $\left(y^{m}\right)_{x}$ opère ainsi à droite sur $\left(J^{m}\right)_{x}$ de façon simplement transitive.

\section{Lemme 3}

Le prolongement holonome d'ordre $m$ de $\Omega, J^{m} \rightarrow \rightarrow v$, possède une structure naturelle de fibré presque principal de base $V$ et d'espace structural $y^{m}$. 


\section{B - Prolongement holonome d'ordre m d'un fibré associé}

Supposons que $\Gamma$ opère différentiablement sur une variété $M$, et soit $E=M_{\Gamma}[?]$ le fibré modelé associé. Par prolongement de l'application $\Gamma \times M \longrightarrow M$, on déduit une application $G^{m} \times\left(_{n}^{m}\right)_{M} \longrightarrow{ }_{n}^{\left(R^{m}\right)}{ }_{M}$ par laquelle $G^{m}$ opère différentiablement à gauche sur ${ }^{\left(\mathbb{R}^{m}\right)_{M}} ; d^{\prime}$ autre part, $L^{m}$ opère aussi sur ${ }^{\left(\mathbb{R}_{n}^{m}\right)}{ }_{M}$, et de telle façon que :

$\lambda<\gamma, m>=\left\langle\lambda(\gamma), \lambda(m)>\quad \forall \lambda \in L^{m}, \forall r \in G^{m}, \quad \forall m \in{ }^{\left(\mathbb{R}^{m}\right.}\right)_{M}$ On se trouve donc dans le cas particulier (K) étudié au paragraphe $I$. Notons $I^{m}(V){ }_{M}$ le fibré modelé $\left(\left(\mathbb{R}^{m}{ }_{n}{ }_{M}\right)_{\left(L^{m}{ }_{n}\right.}\left[\mathrm{H}^{m}(V)\right]\right.$ (dont la fibre en $x$ est l'ensemble ${ }^{I_{x}^{M}(V)} M$ des m-jets de source $x$ d'applications de $V$ dans $M$ ).

\section{Théorème 5}

Le fibré modelé $\left(I^{m}(V) H\right)$
sens de la catégorie $\left.G_{V}\right)^{(y m)}\left[\begin{array}{ll}J^{m} & 3\end{array}\right.$ est canoniquenent isomorphe à $J^{m} E$ (au On définit l'isomorphisme dont il est question, en associant, à la classe d'équivalence modulo $\left(\mathrm{g}^{\mathrm{m}}\right)_{\mathrm{x}}$ d'un élément $\left(\mathrm{j}_{\mathrm{x}}^{\mathrm{m}}{ }^{\mu}, \mathrm{j}_{\mathrm{x}}^{\mathrm{m}} \mathrm{s}\right)$ de $\left(I_{x}^{m}(v) \text { H. }\right)_{x}\left(J^{m} \Omega\right)_{x}$, l'élément $j_{x}^{m}(s(\mu))$ de $\left(J^{m} F\right)_{x}$ (où $s(\mu)$ désigne la section de $\mathrm{E}$ définie par $\mathbf{s}(\mu)(y)=$ l'imace de l'élément $\mu(y)$ de $\|$ 
par le difféomorphisme $s(y): H \stackrel{\sim}{\longrightarrow} E_{y}$.

C - Connexions holonomes d'ordre m que $Q$

En opérant $\left(\mathbb{R}_{n}^{m}\right)_{\Gamma}=G^{(m)}$, le groupe $L_{n}^{m}$ laisse invariant le

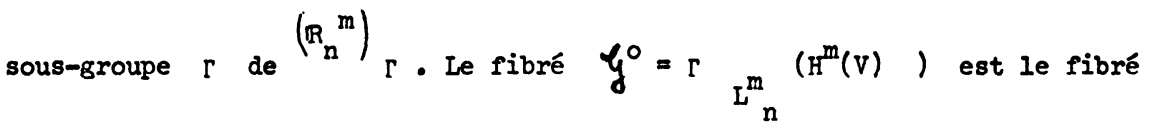
trivial $\Gamma \times v \longrightarrow V: c^{\prime}$ est un sous-espace fibré en groupes de $\xi^{m} \cdot$

\section{Définition 5}

On appellera connexion holonome d'ordre $m$ sur $Q$ tout espace fibré principal $\mathrm{K} \longrightarrow \mathrm{V}$ de eroupe structural $r$, muni d'une structure de sousfibré presque principal de $J^{\mathrm{m}} \mathrm{Q} \longrightarrow \mathrm{V}$.

La restriction à $K$ de la projection canonique $J^{m} \longrightarrow \longrightarrow Q$ étant nécessairement un isomorphisme de fibrés principaux de $\mathrm{K}$ sur $?$,

on peut encore appeler connexion holonome d'ordre $m$ sur $Q$ tout morphisme de $Q$ dans $\mathrm{J}^{\mathrm{m}} \mathrm{Q}$ réalisant $Q$ comme sous-fibré presque principal de $\mathrm{J}^{\mathrm{m}} \mathrm{Q}$.

Equivalence avec la définition d' EHRESMANN

D'après le théorème 4 du paragraphe $I-C$, on peut encore définir une connexion holonome d'ordre $m$ sur $\Omega$, comme une section differentiable $d u$ fibré $\left(y^{m} / y_{0}\right) y^{m}\left[J^{m} Q\right]$. 
Soit $\oint_{x}$ la variété des difféomorphismes $\varphi$ de $q_{x}$ sur $\xi_{y}$ qui vérifient $\varphi(z, \gamma)=\Psi(z) \cdot \gamma \quad \forall z \in \Omega_{x} \quad \forall \gamma \in \Gamma \cdot$ L'ensemble $\Psi_{x}=\bigsqcup_{y \in V} \Phi_{x y}$ est naturellement muni d'une structure de fibré localement trivial de base $V$ et de fibre type $r$. Soit $\mathscr{G}_{x}^{\mathrm{m}}$ la sousvariété de $\left(\mathrm{J}^{\mathrm{m}} \Psi_{x}\right)_{x}$ formée des m-jets des sections $s: V \longrightarrow{ }_{x}$ telles que $s(x)$ soit l'identité de $\eta_{x}$ sur $\xi_{x}$.

Théorème 6

$\mid \begin{aligned} & \text { Il existe un diffeomorphisme canonique } \\ & \zeta_{x}^{m}\end{aligned}\left(\left(y^{m} / y^{\circ}\right) y^{m}\left[J^{m} Q\right]\right) \times$ sur Le fibré $\left(e_{j}^{m} / \xi_{0}\right) y_{m}\left[J^{m} \Omega\right]$ n'est donc rien d'autre que le fibré des éléments de connexion holonome d'ordre $m$ au sens d'EHRESMANH 4 .

Démonstration du théorème 6

Soit $g$ une application $V \longrightarrow \Gamma$, et $\sigma$ une section locale de Q. Au couple $(g, \sigma)$ on associe une section locale $s$ de $\psi_{x}$ ainsi définie : en tout point $y$ où $\sigma$ est définie, $s(y)$ désignera l'isomorphisme de $b_{x}$ sur $Q_{y}$ appliquaic $\sigma(x)$ sur $\sigma(y) . g(y)$. Il est clair que $j_{x}{ }_{x}$ ne dépend que de $\left(j^{m} x^{E}, j_{x}^{m} \sigma\right)$ et même que de la classe d'équivalence de $\left(j^{m}{ }_{x} g, j_{x}^{m}{ }^{\sigma}\right)$ moduro $\left(y^{m}\right)_{x}$. On définit ainsi une application de $\left(y^{m}\right)_{x} \times\left(J^{m}\right)_{x} /\left(y^{m}\right)_{x}$ sur $\left(j^{m} \psi_{x}\right)_{x}$, qui est en fait un difféomorphisme et dont la restriction à $J_{x}^{m} e^{(v, r) \times\left(J^{m} Q\right)_{x} /\left(g^{m}\right)_{x}}=\left(\left(g^{m} / g^{\circ}\right)\left(g^{m}\right)\left[J^{m} Q\right]\right) x$ est un difféomorphisme $\operatorname{sur} \mathscr{G}_{x}^{m}$ (e désigne l'élément neutre de $r$ ), d'où le théorème. 
Equivalence avec les $\mathbb{R}_{1}^{\mathrm{M}}{ }$-connexions au sens de Bourbaki

Rappelons (cf. [l]) que, pour tout fibré principal $? \stackrel{\pi}{\longrightarrow} \mathrm{V}$ de groupe structural $\Gamma$ et pour toute algèbre locale $A$, on munit canoniquement $\mathrm{A}_{0} \stackrel{\mathrm{A}_{\mathrm{N}}}{\longrightarrow} \mathrm{A}_{\mathrm{V}}$ d'une structure de fibré princinal de groupe structural $\mathrm{A}_{\mathrm{r}}$.

D'après Bourbaki, on appelle A-connexion sur $Q$ tout sous-fibré princinal $\mathrm{r}_{1} \longrightarrow \mathrm{A}_{\mathrm{V}}$ de $\mathrm{A}_{\mathrm{O}} \longrightarrow \mathrm{A}_{\mathrm{V}}$, de groupe structural $\Gamma$, possédant en outre les propriétés suivantes :

a) L'inage réciproque de $K_{I}$ par la section canonique $v \stackrel{\sigma_{0}}{\longrightarrow} A_{V}$ de $A_{V}$ est égale au fibré $\mathrm{O} \longrightarrow \mathrm{V}$.

b) $\forall x \in V, \forall z \in \eta_{x}$, il existe une section locale $\sigma$ de $Q$, définie dans un voisinage de $x$, vérifiant $\sigma(x)=z$, et telle que l'application induite $\left(A_{V}\right)_{x} \stackrel{A_{\sigma}}{\longrightarrow}\left({ }^{A} \cap\right)_{z}$ de $I$ 'ensemble des A-points de $V$ proches de $x$ dans l'ensemble des A-points de $\Omega$ proches de $z$, soit un difféomorphisme de $\left({ }^{A} \mathrm{~V}\right)_{x}$ sur la sous-variété $\left(K_{I}\right)_{z}=Y_{I} \cap\left({ }^{A} \cap\right)_{z}$ de $\left({ }^{A}{ }\right)_{z} \cdot[I l$ est clair que la projection $A_{\pi}:\left(V_{I}\right)_{z} \longrightarrow\left(V_{V}\right)_{x}$ définit alors le difféomorphisme inverse ].

\section{Théorème 7}

(i) Ia donnée d'une connexion holonome d'ordre $m$ sur $q$ définit canoniquement une A-connexion sur $?$ pour toute algèbre locale $A$ de rang $\geqslant \mathrm{m}$. 
(ii) Réciproquement, pour $A=\mathbb{R}_{1}^{m}$, toute $\mathbb{R}_{1}^{m}$-connexion sur $Q$ peut être définie ainsi à partir d'une connexion holonome d'ordre $m$ sur $Q$ $d^{\prime}$ une façon et d'une seule.

Soit $\mathrm{K} \longrightarrow \mathrm{V}$ un r-fibré principal, sous-fibré presque principal de $\mathrm{J}^{\mathrm{m}} \mathrm{Q} \longrightarrow \mathrm{V}$. Pour tout point $\mathrm{z}$ de $\Omega$, notons $\mathrm{K}_{z}$ l'image de $z$ par l'isomorphisme canonique de $Q$ sur $K$ : il existe une section locale $\sigma$ de $Q$, définie au voisinage de $x=\pi(z)$, telle que $K_{z}=j_{x}^{m}$ 要 qui est un homomorphisme d'algèbre $I_{z}^{m}(Q) \longrightarrow I_{x}^{m}(V)$. A tout A-point $x^{\prime}$ de $V$ proche de $x\left(x^{\prime}: I_{x}^{m}(V) \longrightarrow A\right)$, associons le A-point de $\Omega$ proche de $z$ écal à $x^{\prime} \cdot K_{z}$. Soit $K_{1}$ la sous-variété de $\left(A_{\Omega}\right)$ formée des éléments $x^{\prime} \cdot K_{z}$, fibrée au-dessus de $A_{V}$ par l'application $x^{\prime} \cdot Y_{z} \longrightarrow x^{\prime}$ (l'application $x^{\prime} \longrightarrow x^{\prime} \cdot K_{z}$ est injective). On vérifie facilenent que $K_{1} \longrightarrow A_{V}$ est un r-sous-fibré principal de $A_{Q} \stackrel{A_{\pi}}{\longrightarrow} A_{V}$ satisfaisant aux propriétés d'une A-connexion.

Réciproquement, soit $\mathrm{K}_{1} \longrightarrow \mathrm{A}^{\mathrm{V}}$ une A-connexion sur $\mathrm{Q}$ : pour tout point $\mathrm{z}$ de $Q$, il existe donc une section locale $\sigma$ de $Q$, définie au voisinage de $x=\sigma(z)$, induisant un difféomorphisme $A_{\sigma}$ de $\left(A_{V}\right)_{x}$ sur $\left(K_{I}\right)_{z}=K_{I} \cap\left(A_{Q}\right)_{z}$.

D'après le lemne 2 (rappels au début de la partie II), le difféomorphisme précédent détermine entièrement $j_{x}^{m}{ }_{x}$ pour $A=\mathbb{R}_{1}^{m}$. Posons alors $K_{z}=j_{x}^{m} \sigma: l^{\prime}$ ensemble $K$ des $K_{z}$ est muni naturellement d'une structure de $\Gamma$ fibré principal, sous-fibré presque principal de $\mathrm{J}^{\mathrm{m}} \mathrm{h} \longrightarrow \mathrm{V}$, et $r$ est l'unique connexion d'ordre $m$ induisant $K_{1}$, d'où le théorème. 
Equivalence avec les surconnexions holonomes d'ordre $m$ au sens de

P. LIBERMAMn (résultat de NGO VAN nUUE [9] )

Soit $\mathcal{R}: \mathrm{r} \longrightarrow G L(M)$ une représentation linéaire de $r$ dans un espace vectoriel $M$ de dimension finie, et soit $E=M Q(\Gamma)[?]$ le fibré vectoriel associé.

Rappelons (cf. [8]) que $\mathrm{J}^{\mathrm{m}} \mathrm{E} \longrightarrow \mathrm{V}$ est un fibré vectoriel différentiable, et que la projection naturelle $\tilde{p}: J^{m_{E}} \longrightarrow E$ est linéaire sur chaque fibre.

On appellera "connexion ( 1 ) holonome d'ordre $m$ sur $E$ au sens de P. LIBERMAN" tout relèvement de $\tilde{p}$, c'est-ã-dire tout homomorphisme (au sens de $F_{\mathrm{V}}$ ), injectif linéaire sur chaque fibre, $\stackrel{z}{i} \mathrm{E} \longrightarrow \mathrm{J}^{\mathrm{m}} \mathrm{E}$, tel que $\tilde{p} \cdot \tilde{l}^{2}=I d_{E}$. IMGO VAN QUE a démontré dans [9] que cette définition équivaut (lorsque $\Gamma=G L(M)$ ) à celle $d^{\prime}$ une connexion holonome d'ordre $m$ au sens $d^{\prime}$ EHRESMAIN. $D^{\prime}$ après le théorème 6 ci-dessous, il revient au même de démontrer le

\section{Théorème 8}

(i) Il existe une application canonique de l'ensemble des connexions holonomes d'ordre $m$ sur $Q$ au sens de la définition 5 , dans l'ensemble des connexions holonomes d'ordre $m$ sur $E$ au sens de P. LIBERMANN

(ii) Si la représentation $R: \Gamma \longrightarrow G L(M)$ est fidèle, l'application précédente est injective.

(iii) Si $\Gamma=G L(M)$ (et si $R$ est l'identité dans $\Gamma$ ), I'application précédente est bijective.

(1) On appelle ici "connexion" (resp. "sous-connexions", ce que P. LIRERRAMN! appelle "sur connexion" (resp. "connexion"). 
Soit en effet $i$ : $Q \rightarrow J^{m} Q$ une connexion holonome d'ordre $m$ sur $Q$. Soit e $\epsilon E_{x}$. Choisissons alors un élément $q$ arbitraire de $Q_{x}$ et un élément $m$ de $M$. (nécessairement unique si $\mathcal{R}$ est fiđèle) tel que e soit égal à la classe d'équivalence $(\overline{q, m})$ de $(q, m)$ modulo $r$. Soit $\sigma$ une section locale de $Q$ telle que $i(q)=j^{m} \sigma(\sigma(x)=q)$, et soit $s$ la section locale $y \rightarrow(\overline{\sigma(y), m})$ de $E(s(y)=e)$. Le jet $j^{m} s$ de cette section ne dépend que de $i$ et $e$, et l'application $\tilde{i}: e \rightarrow j_{x}^{m}{ }^{m}$ ainsi définie est une connexion holonome d'ordre $m$ sur $E$ au sens de P. LIBERMANN, d'où la partie (i). Les parties (ii) et (iii) se vérifient alors sans peine.

2ème Partie : Les fibrés à groupe structural variable

III - Généralisation aux A-prolongements

(d'après N. BOURBAKI)

Soit $R \rightarrow V$ un fibré principal de groupe structural $L$, et

$R: L \rightarrow$ Aut $A$ un homomorphisme de groupes de Lie, de $L$ dans le groupe des automorphismes d'une algèbre locale $A$. En tout point $x$ de $V$, le fibré modelé $A=A_{R(L)}[R]$ admet une fibre $A_{X}$ canoniquement munie d'une structure d'algèbre locale isomorphe (non canoniquement) à A .

Supposant $A$ de rang $m$, et $V$ de dimension $n$, on fait opérer à gauche le produit direct de groupes $L \times I^{m}{ }_{n}$ sur $\left(F_{n}^{m}, A\right)$ en posant :

$<(\lambda, \ell), u>=\mathcal{R}(\lambda) \cdot u \cdot \ell^{-1} \quad \forall \lambda \in I, \quad \forall \ell \in L^{m} \quad \forall u \in H_{n}\left(R_{n}^{m}, A\right)$.

La fibre en un point $x$ de $v$ du fibré modelé

$$
A q=\left(H\left(R_{n}^{m}, A\right)\right)_{\left(L \times L^{m}{ }_{n}\right)}\left[R \otimes H^{m}(V)\right]
$$

est alors égale à l'ensemble des $A_{X}$-points de $V$ proches de $x$. 
Pour tout fibré principal $Q \# V$ de groupe structural $\Gamma$. BOURBAKI appelle Afprolongement de $Q$ le fibré $\mathcal{A}_{Q} \stackrel{\mathcal{A}_{\pi}}{\longrightarrow} \mathcal{A}_{\forall}$ (où $\mathcal{A}$ désigne l'image réciproque par $\pi$ du fibré $\mathcal{A})$ dont la fibre en un $\mathcal{A}$-point $x^{\prime}$ de $V$ proche de $x$ est égale à l'ensemble des $A_{x}$-points de $Q$, proches d'un point de $Q_{x}$, sur projetant sur $x^{\prime}$ par $A_{\pi}$.

Par prolongement de $\mathcal{B}_{0}: \mathrm{L} \rightarrow$ Aut $A$, on déduit un homomorphisme de L' dans le groupe Aut $\left({ }^{A} r\right)$ des automorphismes du groupe de Lie ${ }^{A} r$, et le fibré modelé en groupes $G=\left({ }^{A} \Gamma\right)_{L}[R]$ admet comme fibre en un point $x$ de $v$ le groupe de lie $\left(A_{X}\right)_{\Gamma}$. Notons $G p$ l'image réciproque de $G$ par la projection canonique $\mathrm{p}:$ A $\rightarrow \mathrm{v}$.

\section{Théorème 8}

Le fibré $\mathcal{A}_{Q} \rightarrow{ }^{A b} V$ admet une structure naturelle de fibré presque principal d'espace structural $(G p) \rightarrow$ of .

\section{Définition 6}

On appellera Atconnexion sur $Q$ tout espace fibré principal $K \rightarrow A q$, de groupe structural $\Gamma$, sous-fibré presque principal de $\mathcal{A}_{Q}^{\prime} \rightarrow \mathcal{A}_{T}$, vérifiant en outre les conditions suivantes :

$\left.1^{\circ}\right)$ L'image réciproque de $\mathcal{X} \rightarrow \mathcal{A}$ par le plongement canonique $\sigma_{0}: V \rightarrow A_{f}$ est égale au fibré $Q \rightarrow V,\left(\sigma_{0}(x)\right.$ désigne l'homomorphisme nul de $I_{x}^{m}(V)$ dans $\left.A_{x}\right)$.

$\left.2^{\circ}\right) \forall x \in V, \forall z \in Q_{x}$, il existe une section locale $\sigma$ de $Q$, définie dans un voisinage de $x$, vérifiant $\sigma(x)=z$, telle que l'application $\left(A_{x}\right)_{\sigma: p^{-I}(x)}+\left({ }^{\left(A_{k}\right)_{Q}}\right)_{z}$ soit un difféomorphisme de la variété $p^{-1}(x)=(A)_{x}$ des $d_{x}$-points de $v$ proches de $x$ sur la variété $K_{z}$ des $A_{x}$-points de $Q$ proches de $z$ qui sont dans $\mathcal{K}$. (le difféomorphisme inverse $\mathcal{K}_{z} \rightarrow\left(A_{V}\right)_{x}$ est alors égal à l'application induite par $\left.\mathcal{A}_{\pi}\right)$. 
Cas particulier $A_{0}=I^{m}(v)$

Supposant maintenant $\mathcal{A}_{x}=I_{x}^{m}(V)$ en tout point $x$ de $V$, il existe un plongement canonique $\sigma_{1}$ de $V$ dans $V$ autre que $\sigma_{0}$ : c'est celui qui, à tout point $x$ de $V$, associe l'homomorpnisme identité de $I_{x}^{m}(v)$ sur luimême.

\section{Théorème 10}

(i) L'image réciproque par $\sigma_{1}$ du fibré presque princiapl $\mathcal{N}_{Q} \rightarrow \mathrm{Aq}_{\text {est }}$ égale au fibré presque principal $\left(J^{m} Q\right) \rightarrow V$.

(ii) Pour toute $I^{m}(v)$-connexion $\mathcal{K}$ sur $Q$ (qui est en particulier un sousfibré presque principal de $\mathcal{A}_{Q}^{\prime} \rightarrow \mathcal{A}$ l'image réciproque de $\mathcal{K}$ par $\sigma_{1}$ (qui est en particulier un sous-fibré presque principal de $J^{m} Q \rightarrow V$ ) est une connexion holonome d'ordre $m$ sur $Q$.

Ainsi, toute $I^{m}(V)$-connexion sur $Q$ définit canoniquement une connexion holonome d'ordre $m$ sur $Q$, et définit a fortiori une $A$-connexion pour toute algèbre locale $A$ de rang $\geqslant m$. 
[l] N. BOURBAKI - Prolongements d'espaces fibrés et connexions (IIème Pierre - inédit)

[2] H. CARTAN - Les espaces fibrés analytiques

(Symposium internacional de Topologia algebrica - Mexico 1956)

[3] Ch. EHRESMAMN - Colloque de Géométrie différentielle (Strasbourg 1953)

[4] Ch. EHRESMANN - Sur les connexions d'ordre supérieur (Atti del V cong. del unione math. ital. - Torino 1956)

[5] J. FREMKKLL - Cohomologie non abélienne et espaces fibrés (Bulletin de la Société mathématique de france, t. 85, 1957)

[6] GUILWEMATH-STERRBERG - An algebraic model of transitive differential Geometry (Bulletin of the AMS - vol $70-n^{\circ} I$ (1964) p. 16)

[7] KOBAYASHI - Cananical forms on frame bundles of higher order contact Proceedings of symposia in pure Math, vol III AMS 1961

[8] P. LIBERMANN - Sur la Géométrie des prolongements des espaces fibrés vectoriels (Colloque du CNRS, Grenoble, 1963 Annales de l'Institut Fourier)

[9] IGO VAN QUE - De la connexion d'ordre supérieur (Comptes rendus de 1'Ac. des Sc. Paris, 1964, t. 259, n 13)

[10] SINGERSSTERRBERG - The infinite groups of Lie and Cartan - Part I (The transitive groups) Notes miméographiées du UIT et de l'Université de Harvard (1964)

[11] A. WEIL - Théorie des points proches sur les variétés différentiables (Colloque de Géométrie différentielle, Strasbourg, 1953) 